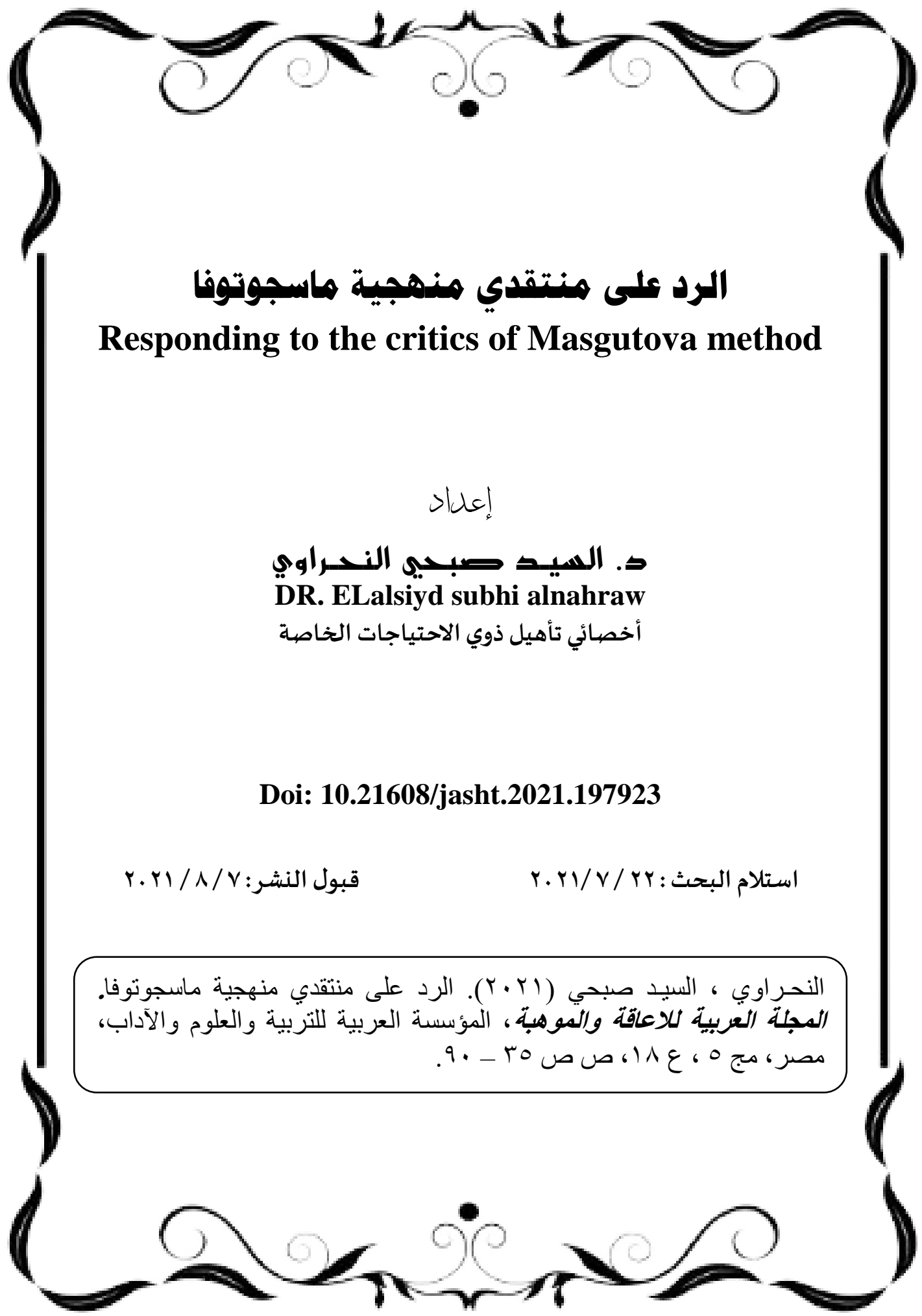




\section{الرد على منتقدي منهجية ماسجوتوفا}

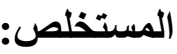

تعتبر منهجية ماسجوتوفا للتكامل العصبي الحسي الحركي للمنعكسات منهجية علمية علاجية تأهيلية، وضعت أصولها وقو اعدها الدكتورة سيفتلانا ماسجوتوفا، أستاذ علم

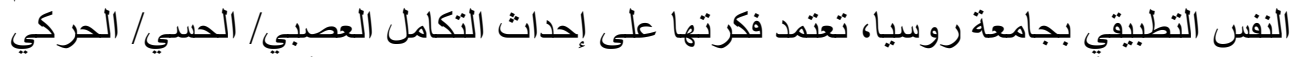

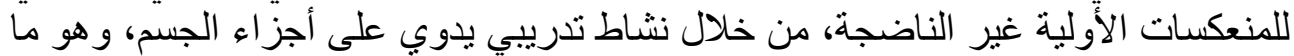
يؤدي إلى تحسُّن في قدر اتهم الحركية و الانفعالية و المعرفية و اللغوية و التعليمية. تقوم الفكرة

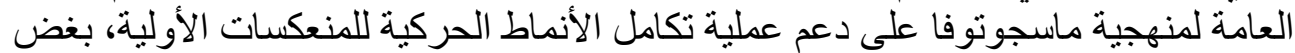
النظر عن حالة الثخص أو عمره. وتعرَّف المنعكسات الأولية على أنها ردود فعل فئل تلقائية

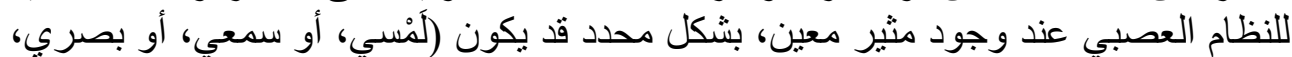

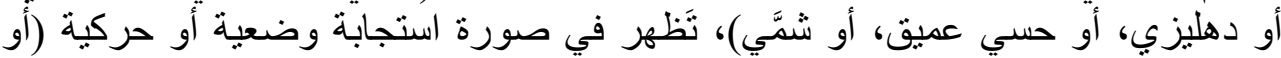
إفراز للغد، أو حركة بؤبؤ العين)، بهدف تنشيطذ النمو العصبي، وهئ، وتفعيل آليات الحماية. والمنعكسات هي أول وأقدم الأنماط الورواثية الحركية السلوكية، والتي يجب دمبن دمجها في قدرات ومهارات حسية حركية عالية التحكم، لتوفير معالجة أو فلك تشفير المدخلات و البرمجة و التحكم في الأعمال الحركية و السلوكية و الذاكرة و التعلم و اللغة و التو اصل. واصله و هكذا فإن أنماط المنعكسات الناضجة تخلق الأساس العصبي الفسيولوجي للتنمية والتعلم؛ في حين أن التطور الضعيف للمنعكسات يؤدي إلى عدم تكامل الحواس، والحركات و الاستجابات الإنيات التفاعلية، والسلوك، و الافتقار إلى التحكم الوضعي، وسودي الته البرمجة و التنسيق الحركي و التي

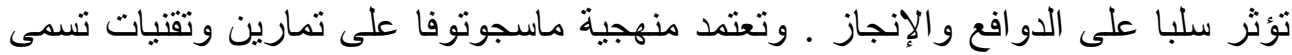

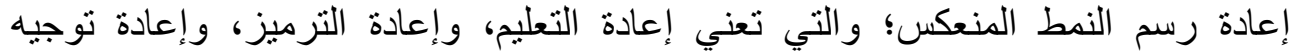

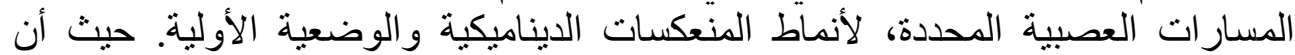

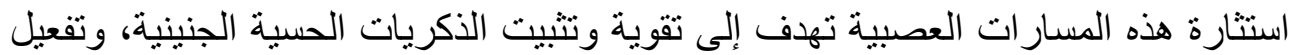
الآليات الدفاعية الفطرية لنظام "إنذار " الجسم في الدماغ . . ومنذ أن دخلت منهجية

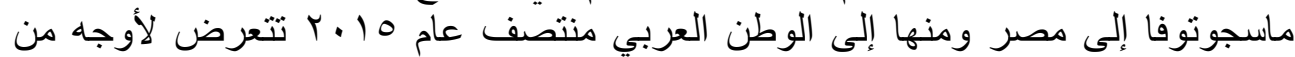

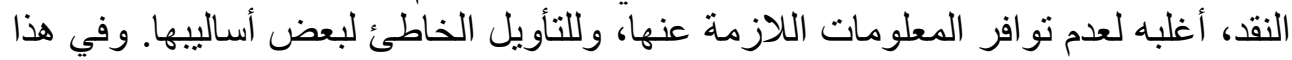
البحث حاولت تفنيد صور النقد بطريقة علمية ومنهجية تستند على رصيد من الخبرة العملية و الممارسة التطبيقية في مجال تأهيل الأشخاص ذوري ذوري الإعاقة.

\section{Abstract:}

The Masgutova Neurosensorimotor Reflex Integration (MNRI®) method is a rehabilitative scientific method, whose origins and rules were developed by Dr. Svetlana Masgutova, Professor of Applied Psychology at the University of Russia. Through a manual 
training activity on the body parts, which leads to an improvement in their motor, emotional, cognitive, linguistic and educational abilities. The general idea of Masgotova's method is to support the integration of motor patterns of primary reflexes, regardless of the person's condition or age. Primary reflexes are defined as the automatic reactions of the nervous system to the presence of a specific stimulus, specifically (tactile, auditory, visual, vestibular, deep sensory, or olfactory), appearing in the form of a postural or motor response (or secretion of the gland)., or pupil movement), with the aim of stimulating neurodevelopment, and activating protective mechanisms. Reflexes are the first and oldest of the behavioral motor genotypes, which must be integrated into highly controlled sensorimotor abilities and skills, to provide processing or decoding of input, programming, control of motor and behavioral actions, memory, learning, language and communication. Thus, mature reflex patterns create the neurophysiological basis for development and learning; While the poor development of reflexes leads to a lack of integration of the senses, movements and interactive responses, behavior, a lack of postural control, poor programming and motor coordination that negatively affect motivation and achievement. Masgotova's methodology relies on exercises and techniques called relocation or pattern redrawing; Which means the re-education, recoding, and redirection of specific neural pathways, of dynamic and postural reflex patterns. As the excitation of these neural pathways aims to strengthen and stabilize embryonic sensory memories, and activate the innate defense mechanisms of the body's "alarm" system in the brain. Since Masgotova's method entered Egypt and from there to the Arab world in mid-2015, it has been subject to criticism, mostly for the lack of necessary information about it, and for the misinterpretation of some of its methods. In this research, I tried to refute the forms of criticism in a scientific and methodical way, based on a balance of practical 
experience and applied practice in the field of rehabilitation of persons with disabilities.

مقدمة

ينطوي نقد منهجية ماسجوتوفا (لمن نقدها) على كثير من الأغاليط المنهجية؛ أي طريقة النقد

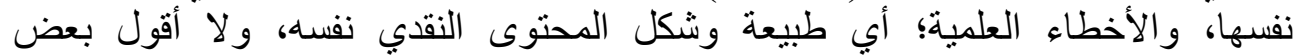
التجاوزات التي لا تليق أيضاء.

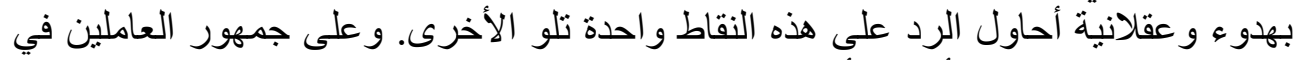

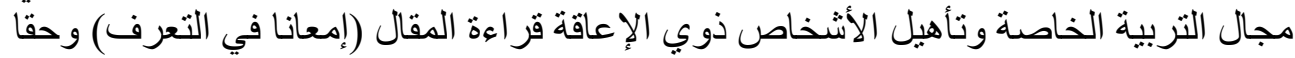

قو اعد تأسيسية

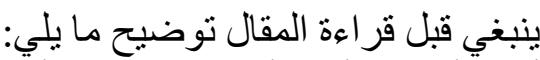

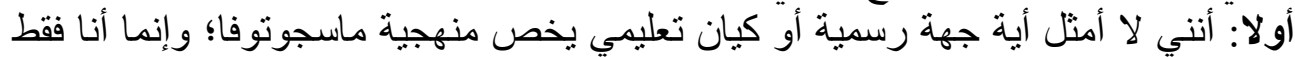

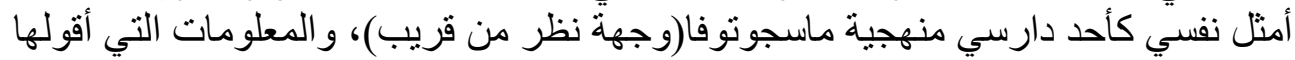

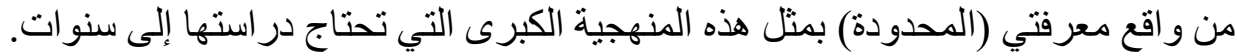

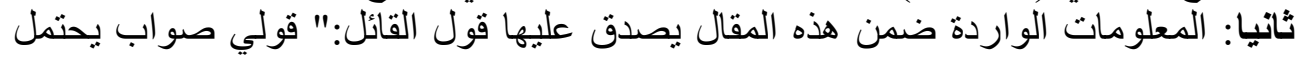

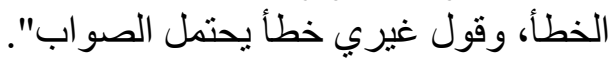

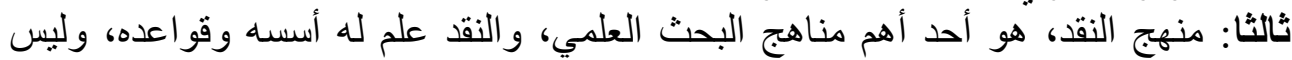

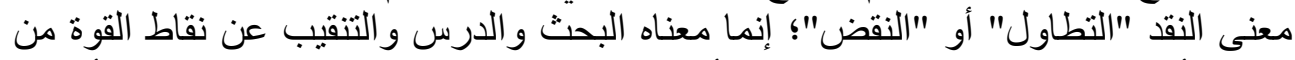

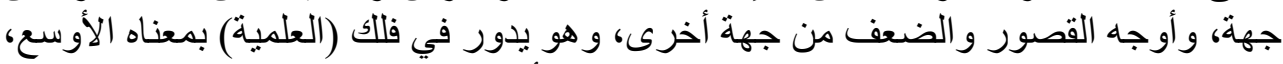

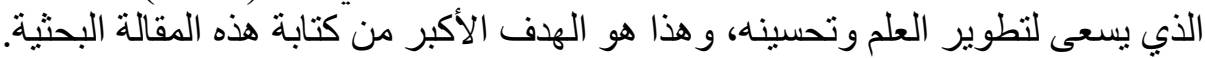

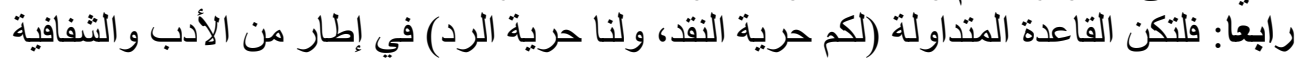

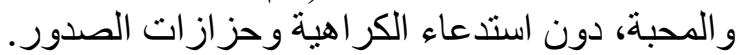

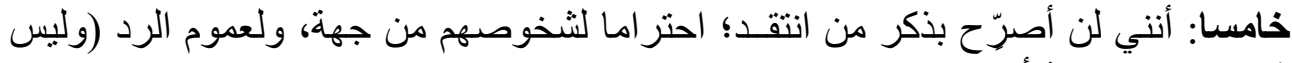
شخصنته من جهة أخرى).

المنهج

يتبني الباحث في هذه المحاولة العلمية فنيات وأساليب المنهج النقدي؛ حيث أنه من

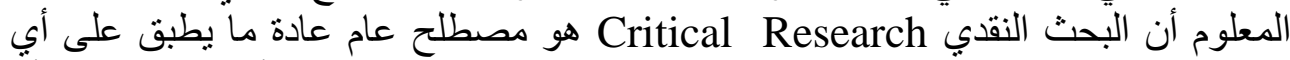

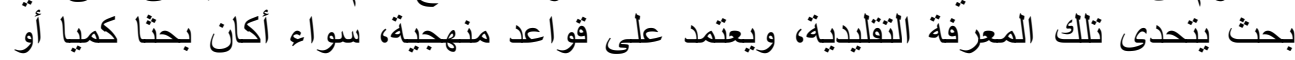
كيفيا، والتي تطبق الموضوعية العلمية (Jupp, Victor. 2006). ويعتمد البحث النقية النقدي

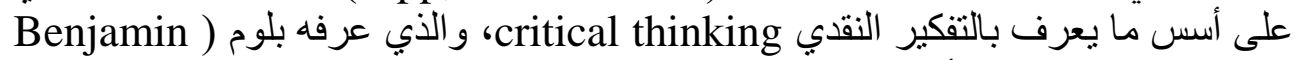

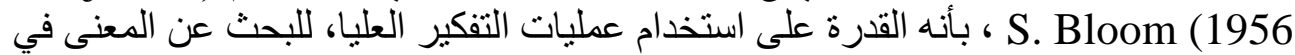
الأعمال أو الأقوال. و عرف زمائه الفئه "المستويات العليا من التفكير بأنها تشمل: التطبيق، 


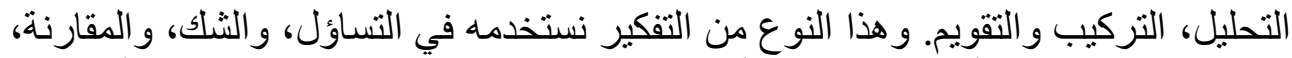

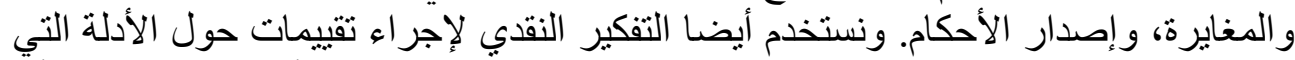

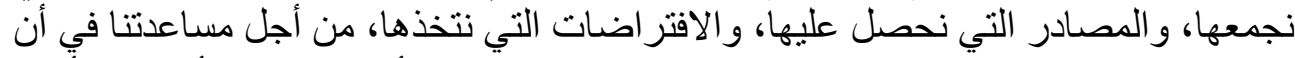

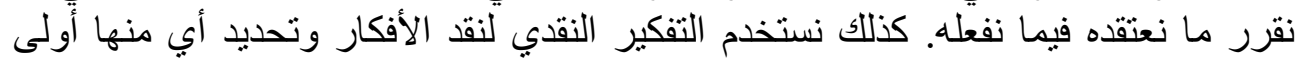

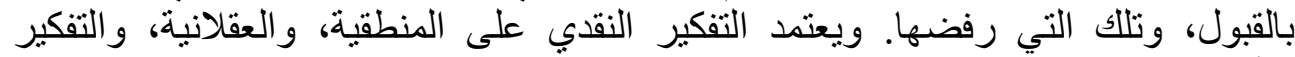

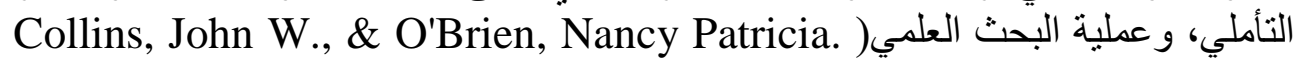
والاتجاه النقدي في التربية إلى جانب أنه يقدم لنا جملة من الأفكار البديلة حول

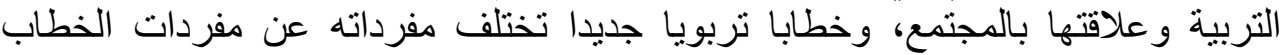

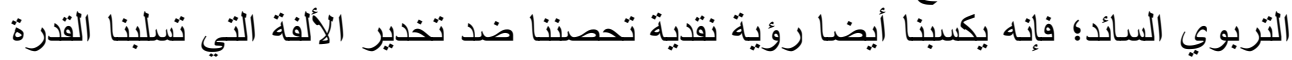

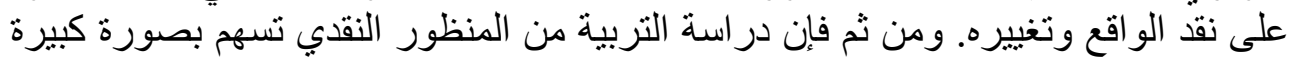

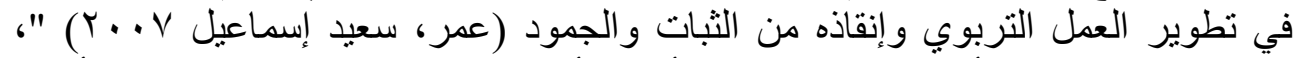

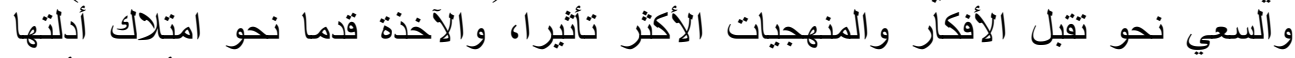

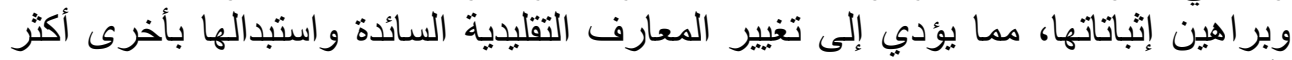
تأثير ا وفاعلية.

منهجية الرد أبرد كي أقوم بكتابة هذا المقال اتبعت الخطوات التبالية:

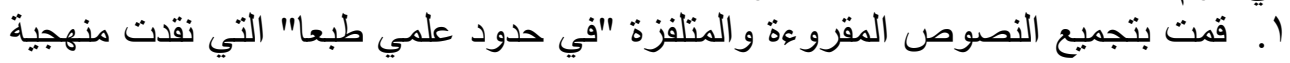
ماسجوتوفا.

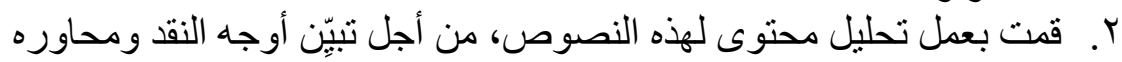

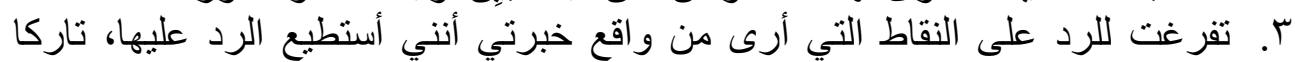

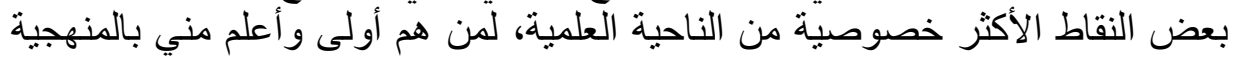

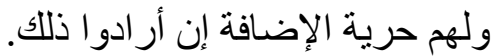
ع. قمت بصياغة الردود وفق آلية، حاولت أن أن يتوفر فيها السلاسة والمرونة، ليسهل

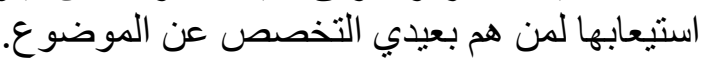

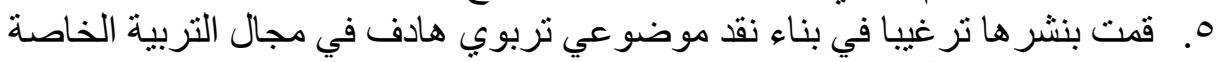
قواعد النقد المغلوطة يتخذ الثخص الناقد لمنهجية ماسجوتوفا عدة خطوات ييني على أساسها: نقده، وتجريم الثخص الذي يقع النقد عليه، وتهييج الجماهير ضده، واستعداء الجهات الرسمية ضداء و إليكمّ (متلازمة النقا) التي طرحها الناقد، والتي تمضي خطواتها في خمس خطوات يَصنع متعاقبة: 
أولا: وضع القاعدة: و هي أن دارسي المنعكسات، ومن يعالجون بالمنعكسات لابد وأن يكونوا

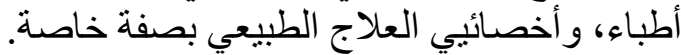

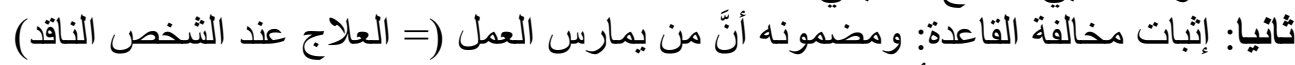

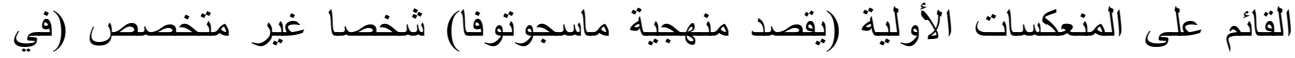
(الطب). ثالثا: تجريم من خالف القاعدة: بعد أن تم وضع القاعدة التأسيسية لدى العامة، و إثبات مخالفة

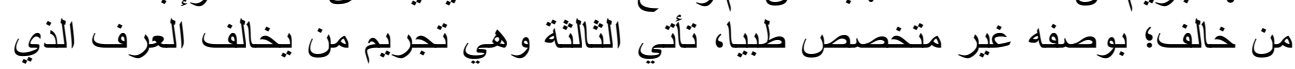
اتفق عليه سلفا. رابعا: استثارة الجماهير وكسب التأييد، من أنه ما يتكلم إلا للمصلحة العامة، وبأنه حريص في التهاء

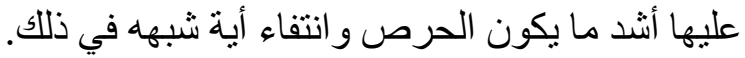

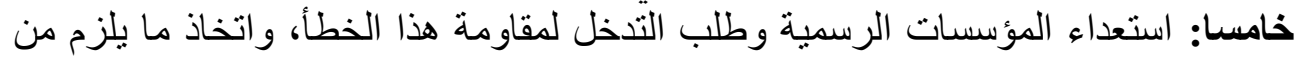

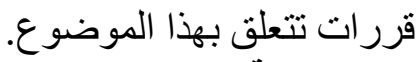

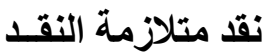

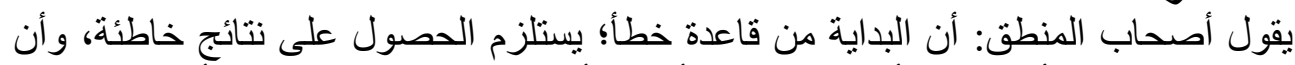

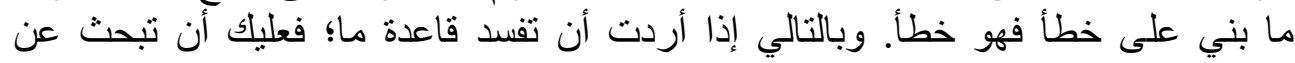
مسلماتها و افتر اضياتها. و هنا تساؤل:

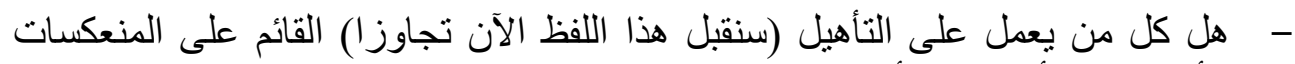

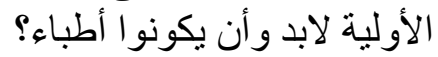

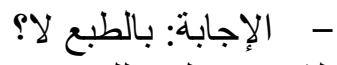
الثنو اهد على ذللك: نحن نعلم جميعا أن عشر ات بل مئات من أخصائيي التكامل الحسي يدرسون المنعكسات؛ بل إن

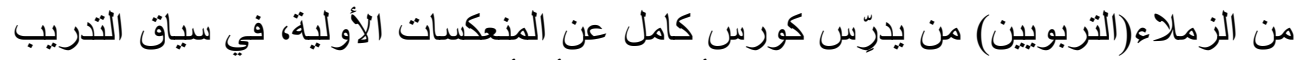

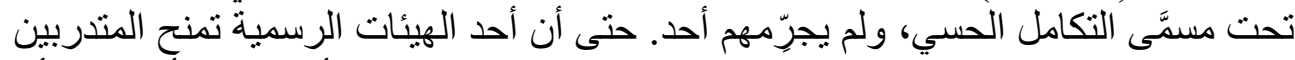

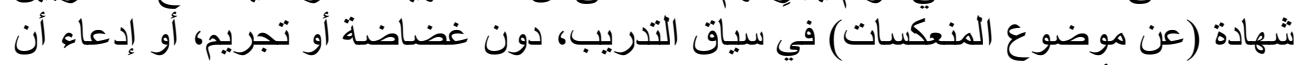

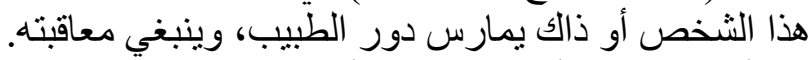

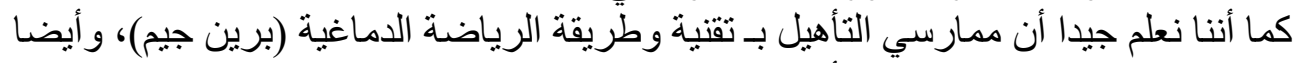

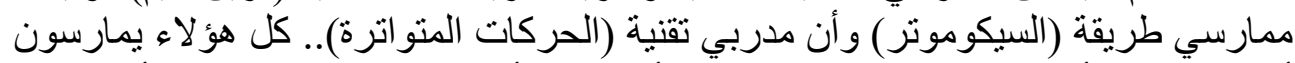

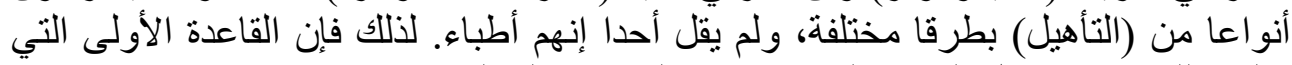

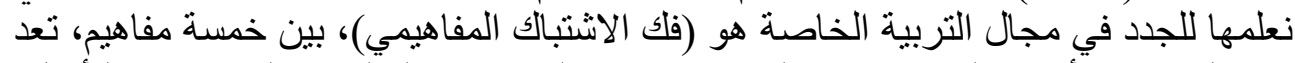

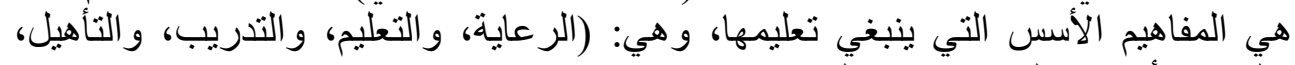
و الَعلاج).أما عن الفرق بينها (على نحو موجز): 
فالر عاية: على أبسط معانيها تعني تقديم خدمة؛ بمعنى أن أبسط ما يقوم به الأخصائي

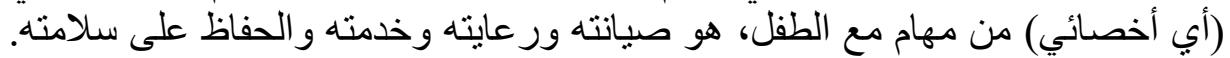

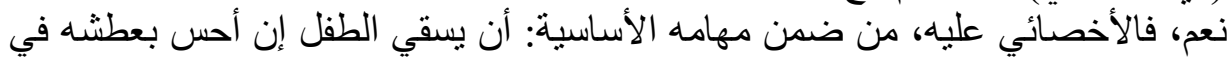

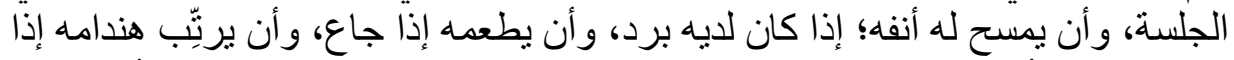

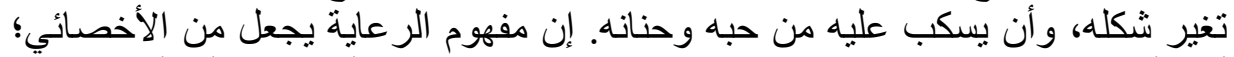

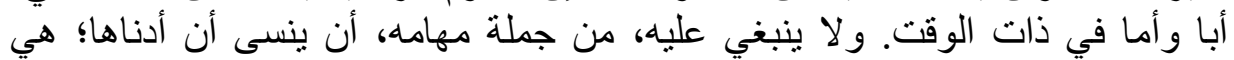

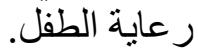

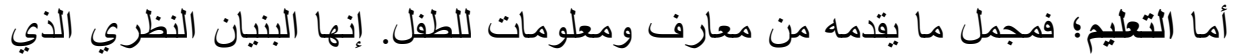

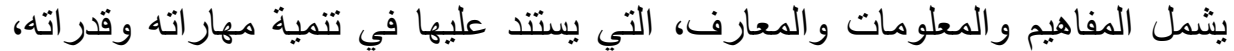

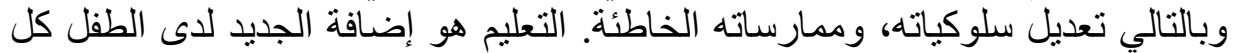

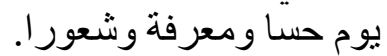
أما التدريب؛ فيعني تللك المهار ات المكتوف المكبة التي يحرص الأخصائي على تعليمها الطفل.

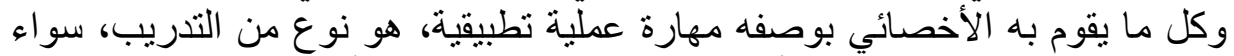

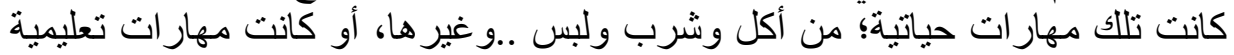

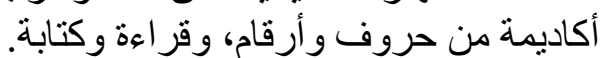

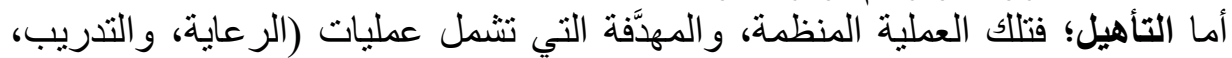

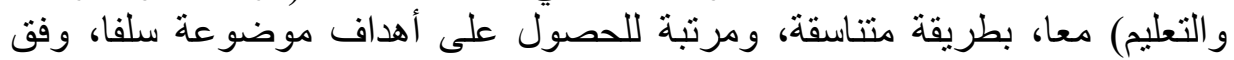

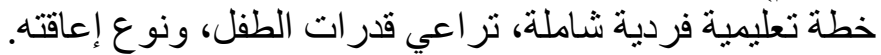

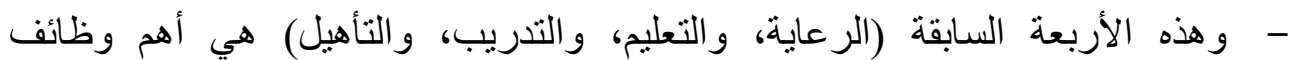

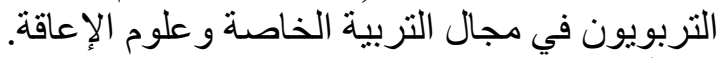

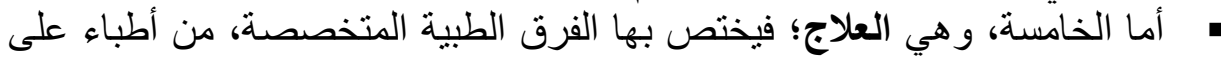

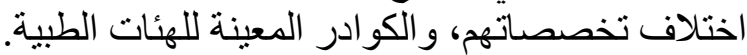

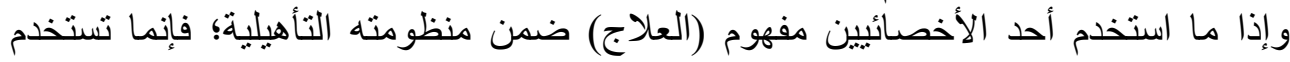

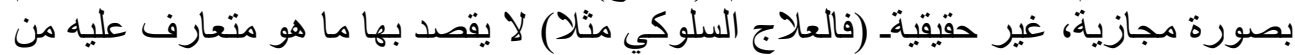

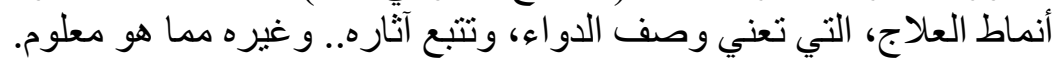

مغالطــة العلاجة

إن المغالطة الحقيقة التي وقع فيها الثخص الناقد لمنهجية ماسجوتوفا أنه أقام تعادلا وتساويا

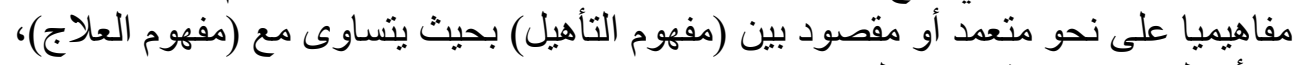

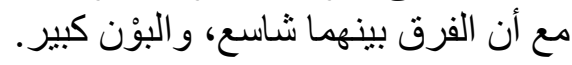

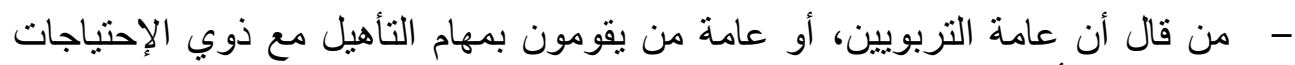
الخاصة هم أطباء؟ أن عامج 


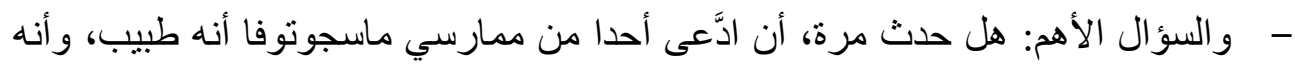

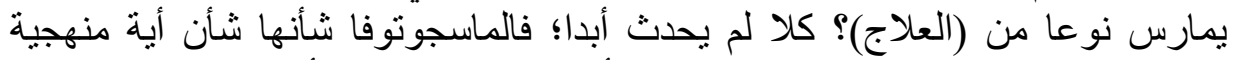

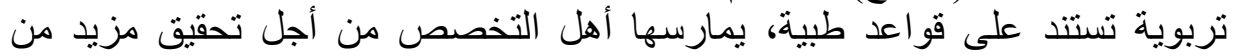

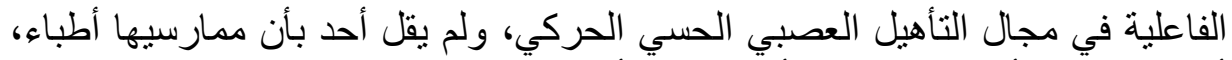

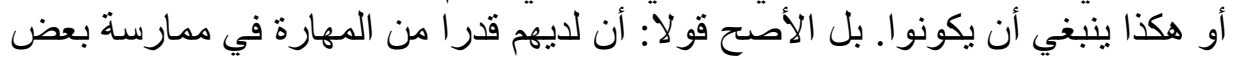

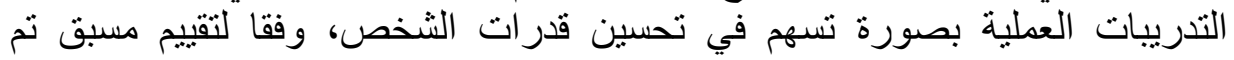

$$
\text { التدريب عليه. }
$$

محاور التشكيك وموضوعاته التهبه

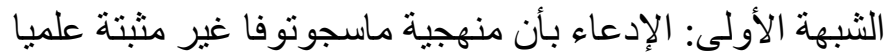

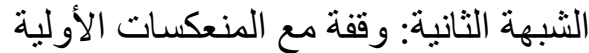

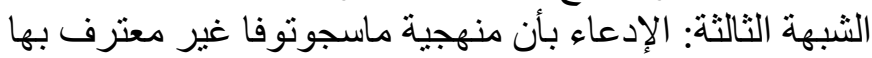

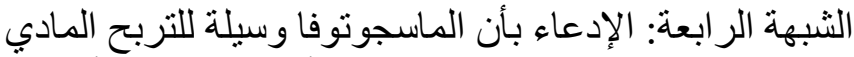

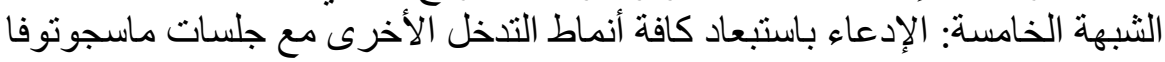

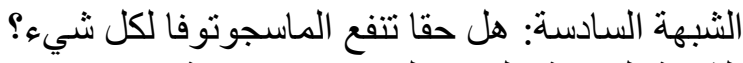

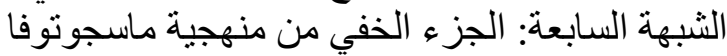

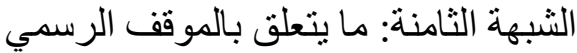
الثبهة التاسعة: طريقة النقا والثبة وأخلاقياته

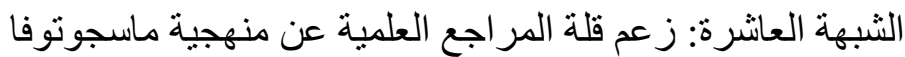

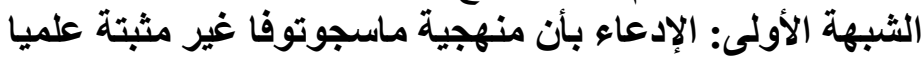

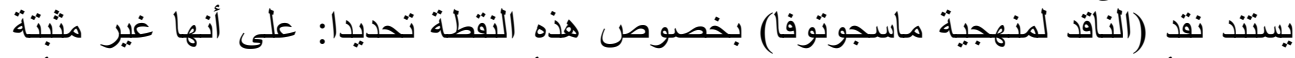

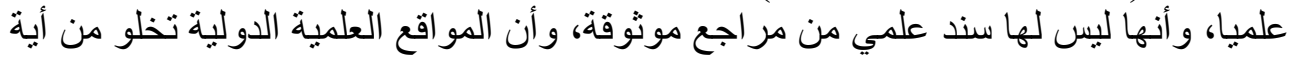

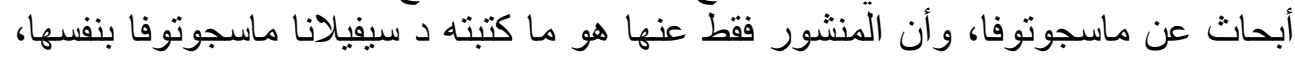

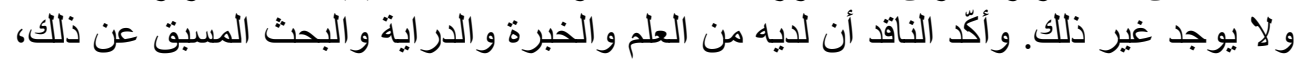

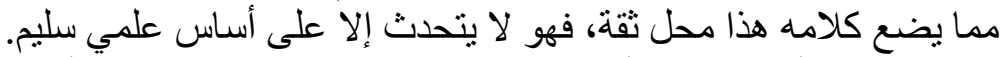

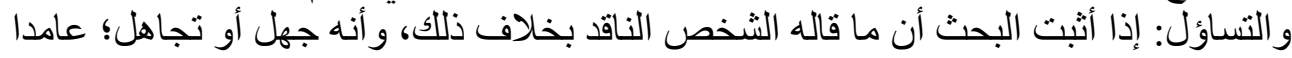

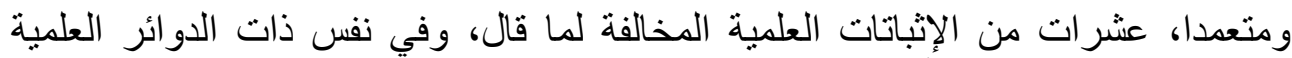

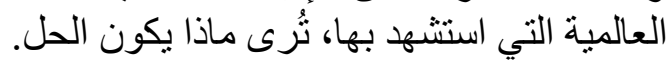
إليك عزيزي القارئ الدر اسات التالية:

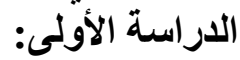
• - العنوان باللغة الإنجليزية:

\section{The Impact of MNRI Therapy on the Levels of} Neurotransmitters Associated with Inflammatory Processes 
ترجمة العنوان باللغة العربية: تأثير العلاج بمنهجية ماسجوتوفا على مستويات الناقلات العصبية المرتبطة بالعمليات الالتهابية التعبية الموقع الذي نشر عليه البحث هو موقع (PubMed@) و هذا هو الرابط الخاص بالها: https://pubmed.ncbi.nlm.nih.gov/

و هو نفس الموقع الذي ذكر مرارا صاحب النقد أنه لا يوجد أي بحث علمي منشور عليه

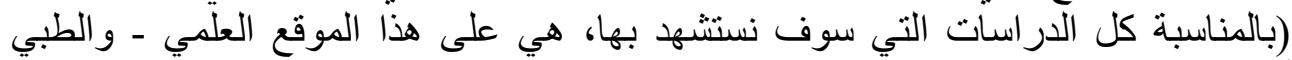

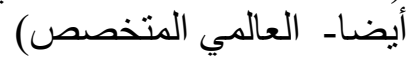
التعريف بهذا الموقع

(بالإنجليزية: PubMed هو محرك بحث مجاني من أجل الوصول إلى قاعدة بيانات

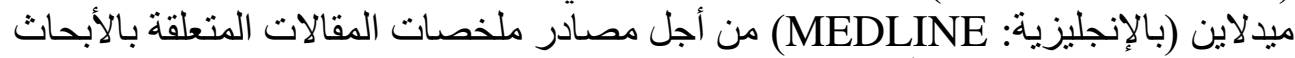

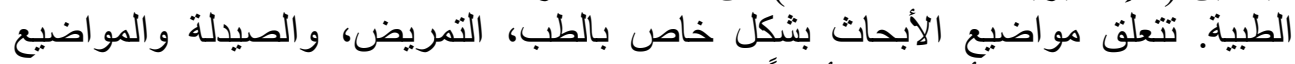

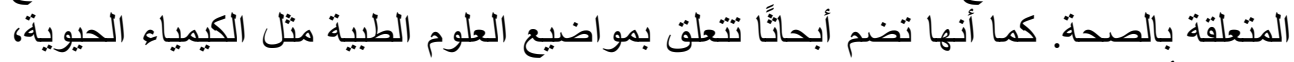

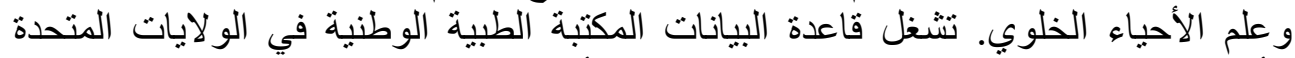

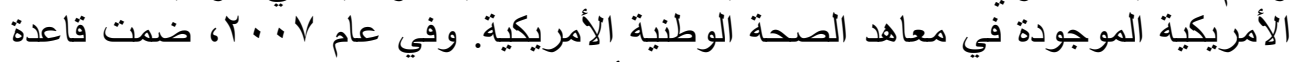

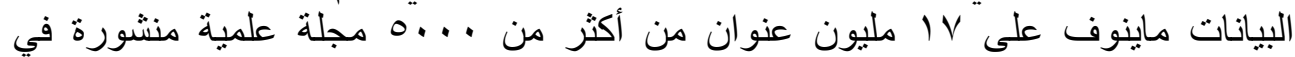

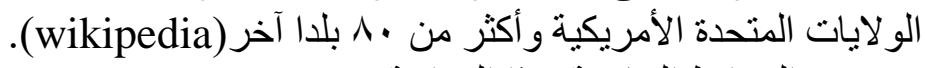

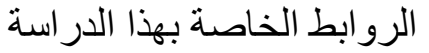

https://pubmed.ncbi.nlm.nih.gov/32085403/ https://www.ncbi.nlm.nih.gov/pmc/articles/PMC7072967/

$$
\text { العلماء المشاركين في هذه الدراسة هم: }
$$

Tatiana V Tatarinova, Trina Deiss, Lorri Franckle , Susan Beaven , Jeffrey Davis مع ملاحظة أن الدكتورة ماسجوتوفا ليست واحدة ممن ألَّف هذا البحث، وهو ما يكسبه مصداقية.

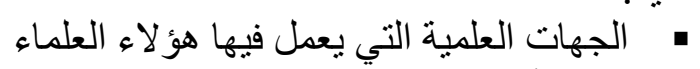

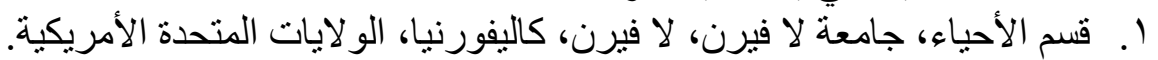

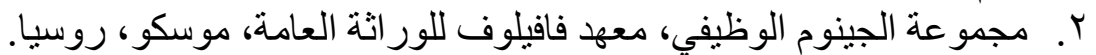

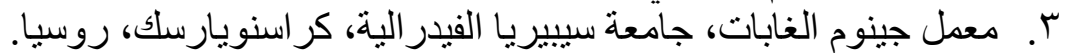

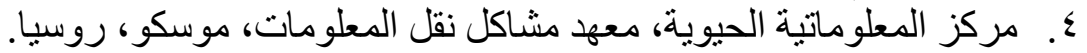

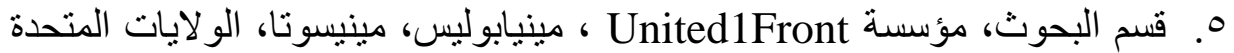

$$
\text { الأمريكية. }
$$

7. قسم صحة الليزر، ليزر هيلث، أور لاندو، فلوريدا، الو لايات المتحدة الأمريكية. 
V. طب الأسرة، عيادة سانت بطرسبرغ الحرة، سانت بطرسبرغ، فلوريدا، الولايات المتحدة الأمريكية.

‘KS ،Wichita ‘Prairie Health and Wellness ‘Family Medicine .^ الو لايات المتحدة الأمريكية.

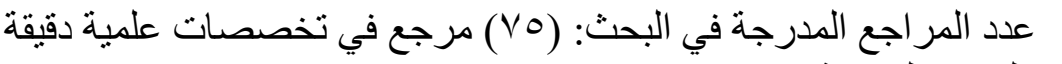

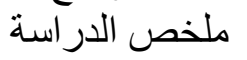

تم اختبار مستويات الناقل العصبي لممثلين من خمس مجمو عات تشخيص مختلفة قبل وبعد

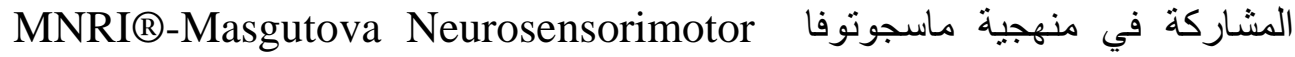
.Reflex Intervention

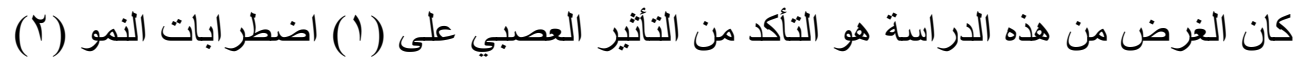

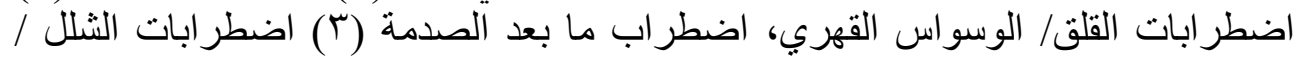

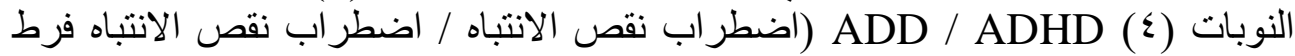

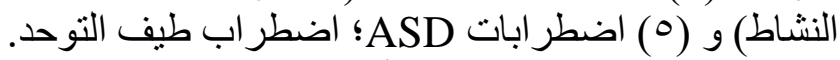

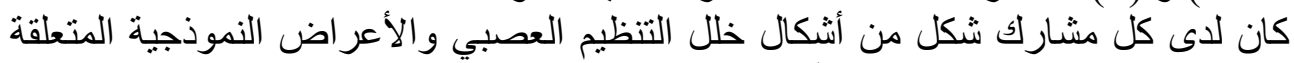

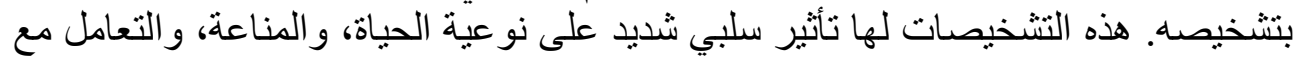

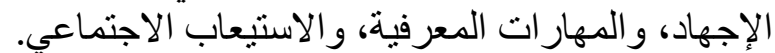

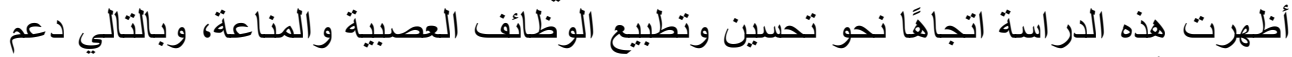

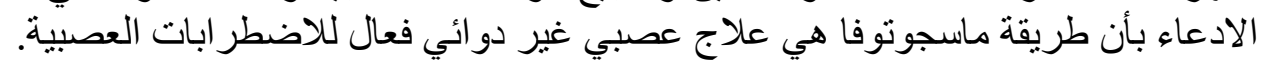

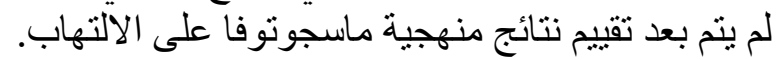

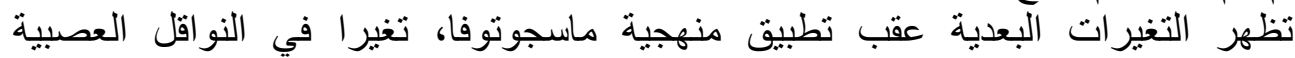

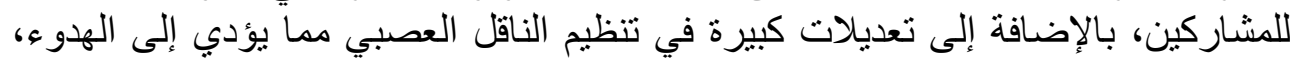

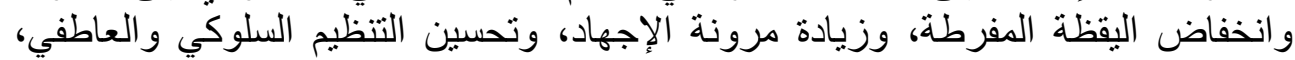

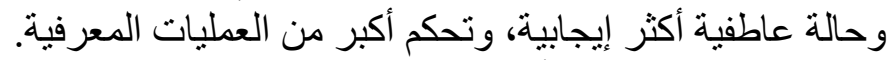

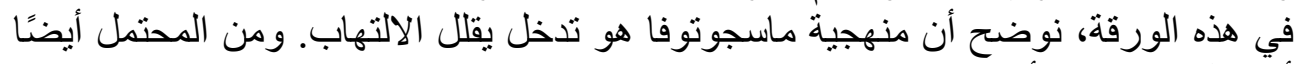

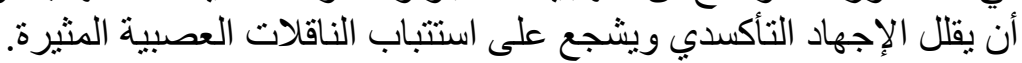

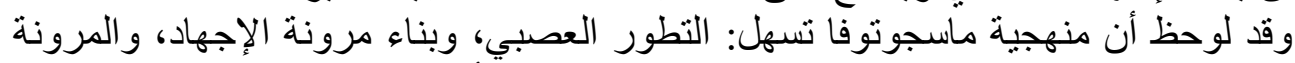

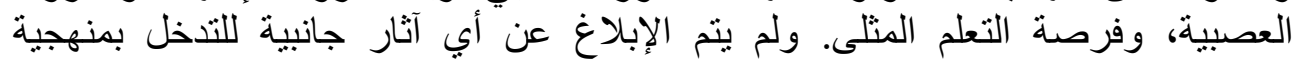
ماسجوتوفا. 


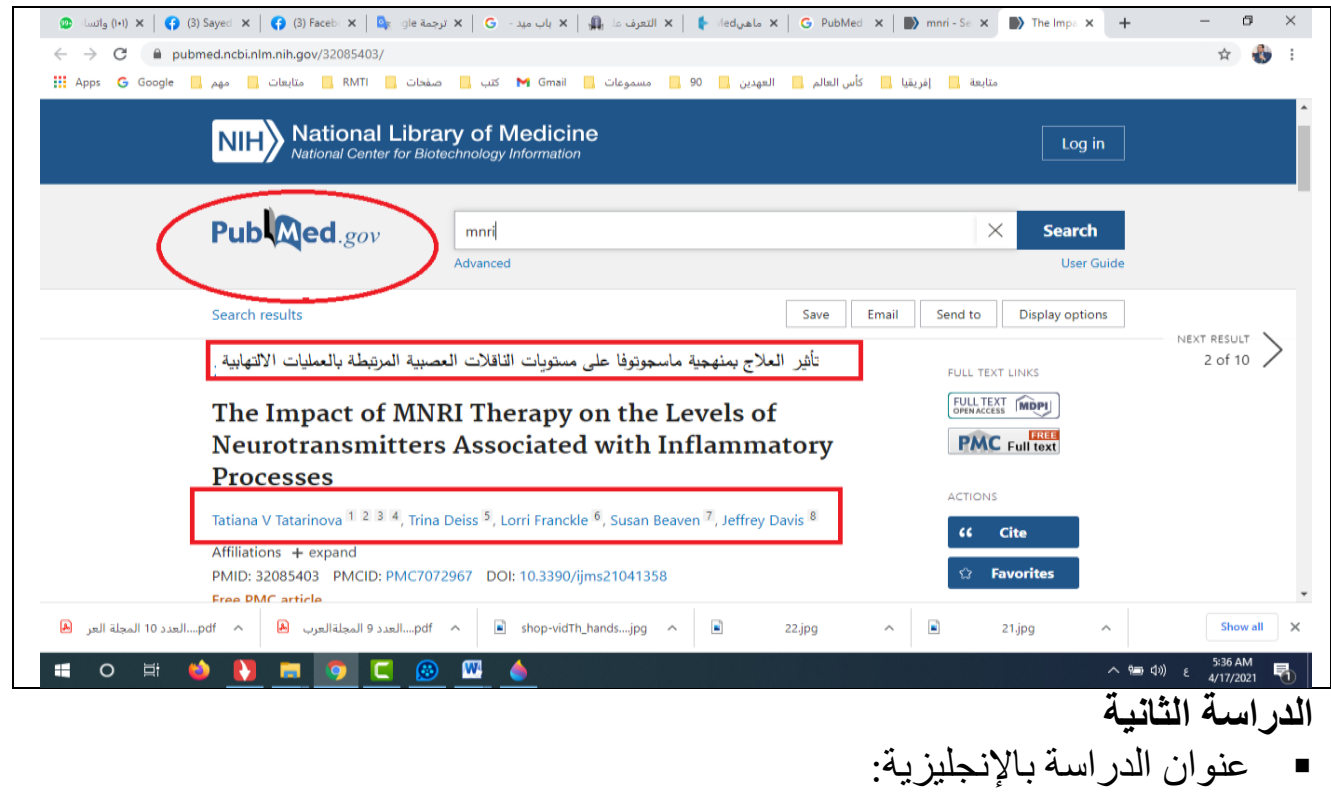

The impact of rehabilitation carried out using the Masgutova Neurosensorimotor Reflex Integration method in children with cerebral palsy on the results of brain stem

\section{auditory potential examinations}

https://pubmed.ncbi.nlm.nih.gov/23214200/

$$
\text { الر ابط الخاص بهذه الدر اسة هو: }
$$

ترجمة العنوان: تأثثر إعادة التأهيل الذي يتم إجراؤه باستخدام منهجية ماسجونوفا في الإني

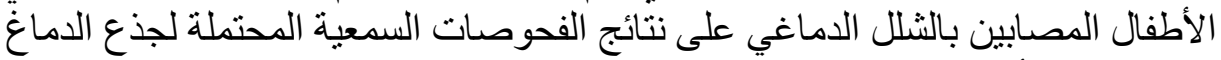

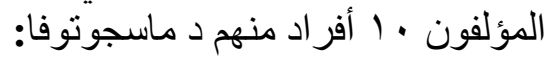

Witold Pilecki, Swietłana Masgutova Joanna Kowalewska, Denys Masgutov, Nelly Akhmatova, Małgorzata Poreba, Małgorzata Sobieszczańska, Piotr Koleda, Anna Pilecka, Dariusz Kałka العمل: يعملون جميعهم في قسم الفيزيولوجيا المرضية، جامعة فروتسواف الطبية ،

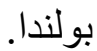
الهدف: كان الهدف من هذه الدراسة هو تحديد كفاءة إعادة التأهيل التي تم إجراؤها

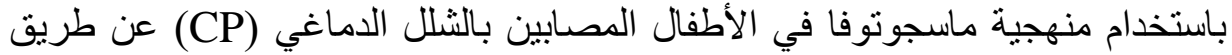
القياسات الموضو عية باستخدام إمكانات فحص جذع الدماغ السمعي المحفز (BAEP). 


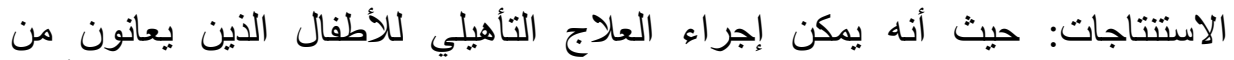

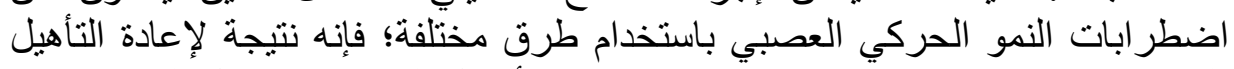

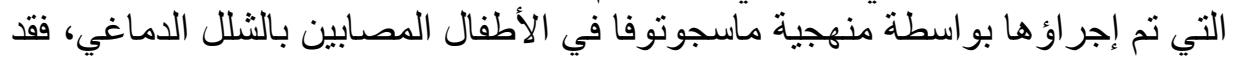

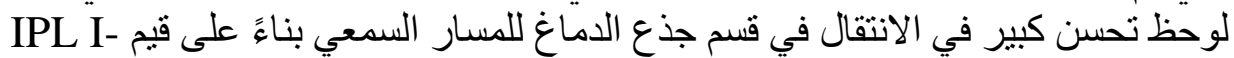
V المطلقة والنسبية. ومع ذللك، كُان التغيير الذي تم التئ الحصول عليه في الأطفال مختلفًا.

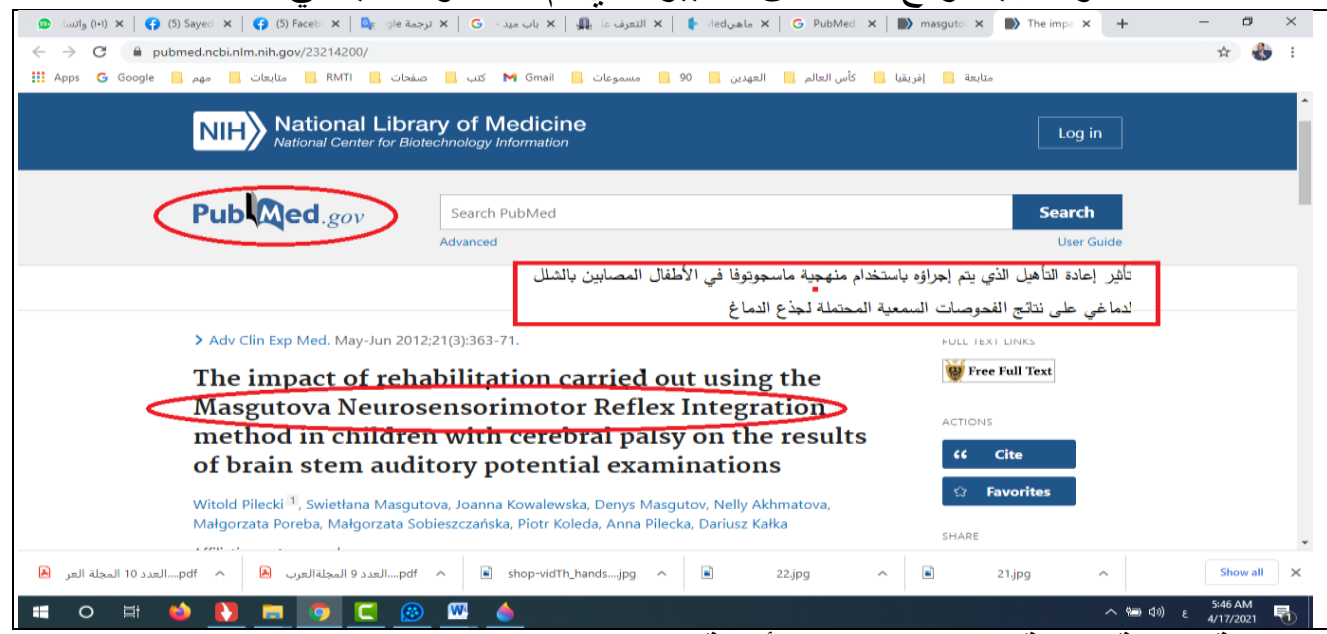

الثبهة الثانية: وقفة مع المنعكسات الأولية

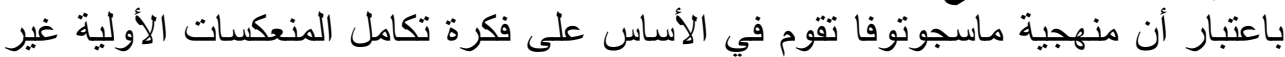

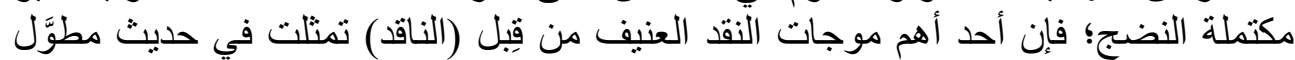

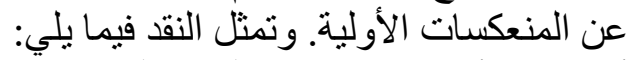

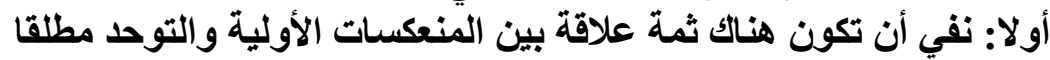

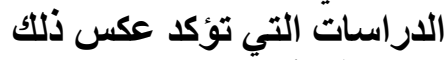

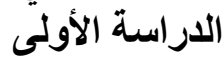

عنوان الدر اسة بالإنجليزية:

Persistent primary reflexes affect motor acts Potential implications for autism spectrum disorder

$$
\text { الر ابط الخاص بهذه الدر اسة: }
$$

/https://pubmed.ncbi.nlm.nih.gov/27595468

العنوان بالعربية: تؤثر المنعكسات الأولية التي ما زالت نشطة على الأداء الحركي:

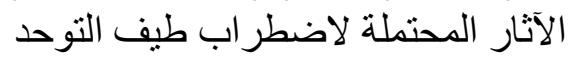

المؤلفون: Alice Chinello , Valentina Di Gangi , Eloisa Valenza 
الجهات العلمية التي يعطلون بها:

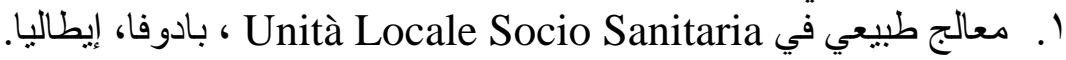

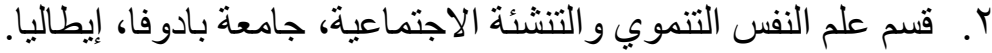

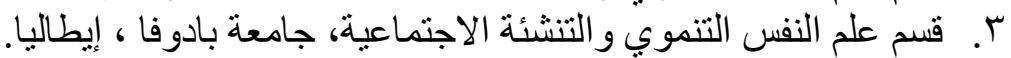

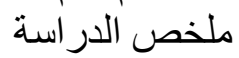

في التطور الحركي النموذجي؛ بعتمد التقدم في استخدام الإجراءات الموجهة نحاء التحو الهدف

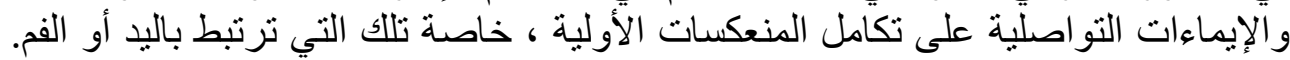

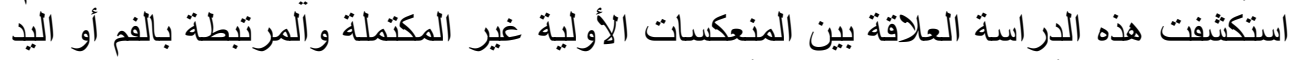

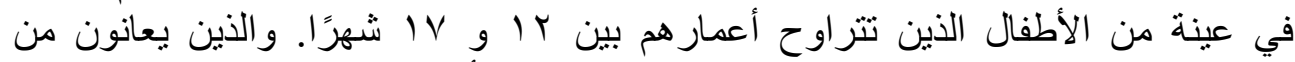
اضطر اب طيف التوحد، وغالبًا ما يو اجهون صعوبة التعار في أداء الحركات الدقيقة، ويظهرون

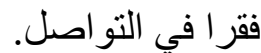
أوضحت النتائج أن المنعكسات الأولية غير مكتملة النضج، مرتبطة بالمخزون التركي التركي

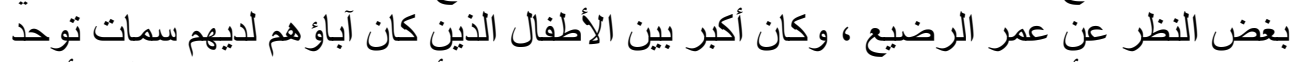

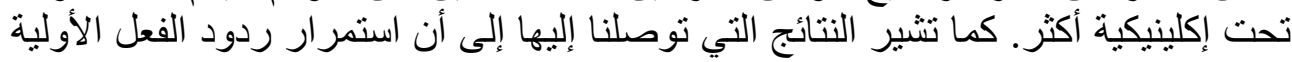

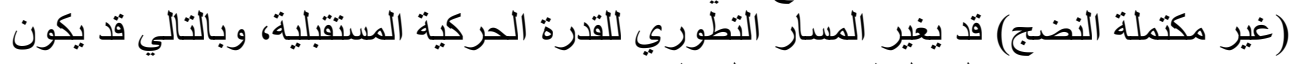
تقييمها مؤشرًا مبكرًا على التطور غير النير النمطي.

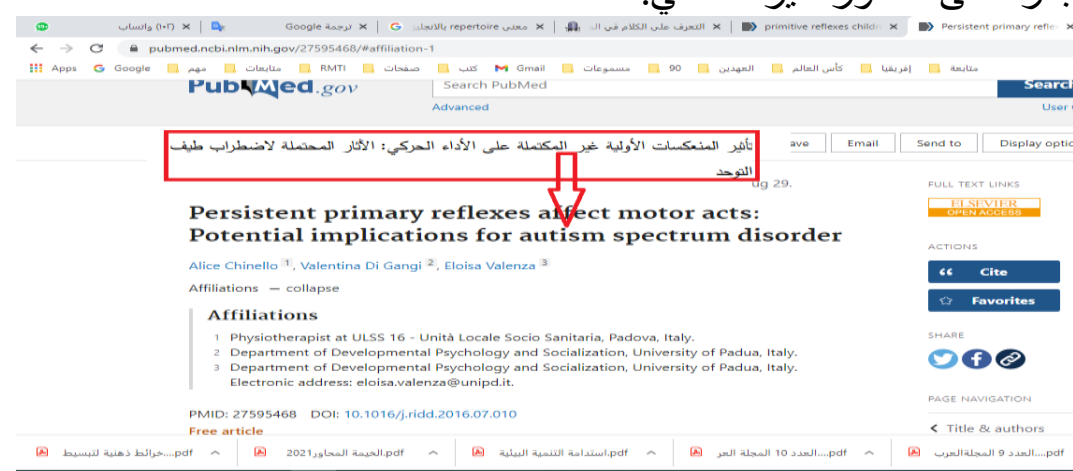

\section{Primitive reflexes in very low birth weight infants later diagnosed with autism spectrum disorder} الر ابط الخاص بهذه الدر اسة: /https://pubmed.ncbi.nlm.nih.gov/32549029 ترجمة العنوان: المنعكسات الأولية عند الرضع منذفضي الوزن عند الولادة الذين تم

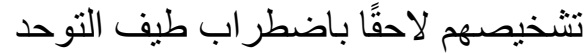


المؤلفون، ثلاثة من العلماء اليابانيون هم: Yukiyo Nagai , Kayo Nomura Osamu Uemura تخصصاتهم العلمية: ا. قسم طب الأطفال، مستشفى ناغويا دايني التابع للصليب الأحمر الياباني، آيتشي، اليابان

Y. قسم طب الأطفال، مستشفى ناغويا دايني للصليب الأحمر الياباني، آينثي ، اليابان.

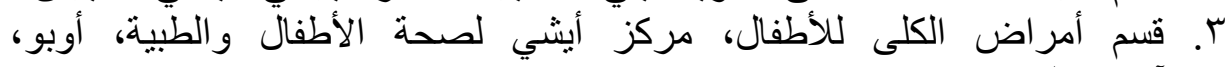
آيتشي، اليابان.

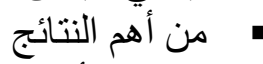

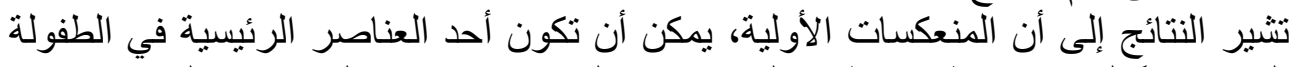

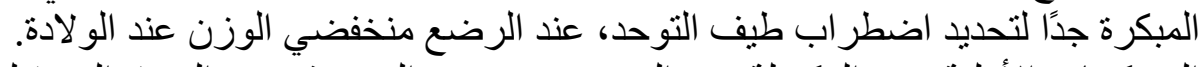

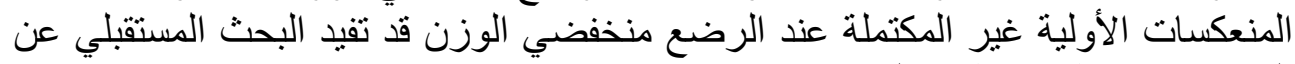

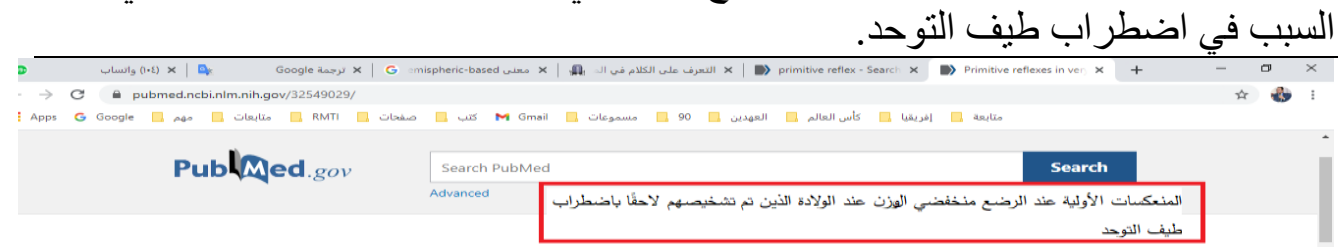

> Minerva Pediatr. 2020 Jun 16. doi: 10.23736/50026-4946.20.05784-9. Online ahead of print.

Primitive reflexes in very low birth weight infants later diagnosed with autism spectrum disorder

Yukiyo Nagai ${ }^{1}$, Kayo Nomura ${ }^{2}$, Osamu Uemura ${ }^{3}$

Affiliations + expand

PMID: 32549029 DOI: $10.23736 / 50026-4946.20 .05784-9$

Abstract

Background: As early screening and diagnosis is very important in treatment and intervention of

Autism Spectrum Disorder, we investigated the relationship between primitive reflexes and Autism

$$
\text { السبب في اضطر اب طيف التوحد. }
$$

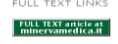

Actions

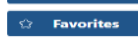

SHARE $\oplus \boldsymbol{Q}$

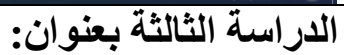

\section{The potential role for emergence in autism}

$$
\text { الر ابط الخاص بهذه الدر اسة: }
$$

https://onlinelibrary.wiley.com/doi/pdfdirect/10.1002/aur.2

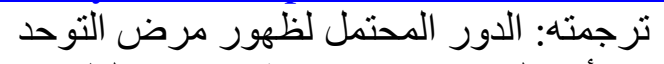

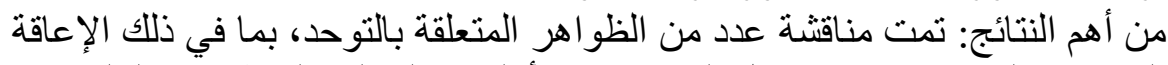

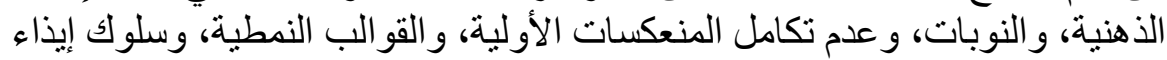

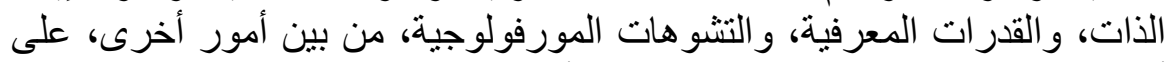

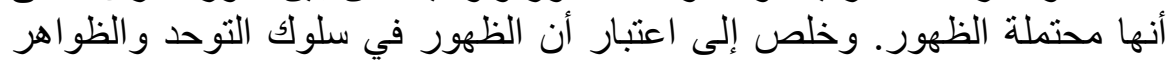


ذات الصلة يجب أن تكمل النهج الاختزالي وقد تساعد في إلقاء الضوء على

$$
\text { مكونات وتعقيدات التوحد. }
$$

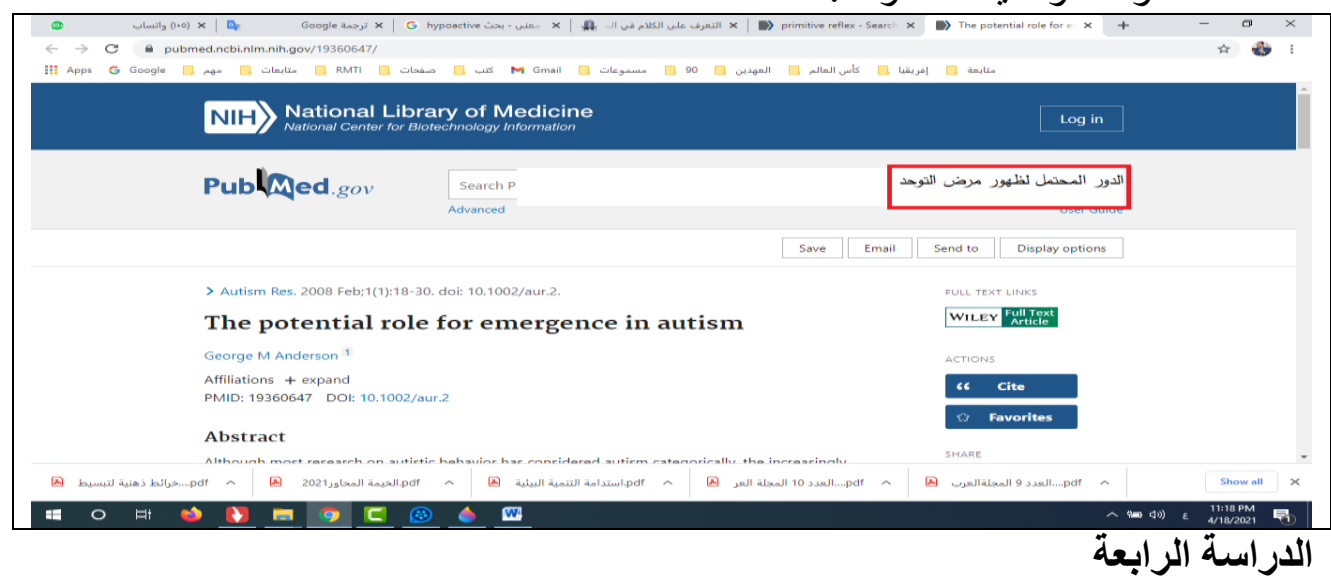

Psycho-Cognitive Intervention for ASD from Cross-Species Behavioral Analyses of Infants, Chicks and Common • الرابط الخاص بملخص هذه الدراسة https://www.ingentaconnect.com/contentone/ben/cnsnddt/2016/00 $\underline{000015 / 00000005 / \operatorname{art} 00010}$ /https://pubmed.ncbi.nlm.nih.gov/27071788 • العنوان: التدخل النفسي المعرفي لاضطر اب طبف التوحد عبر التحليلات السلوكية

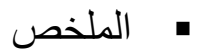

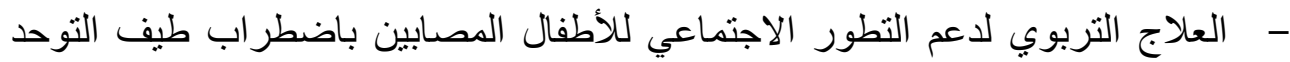

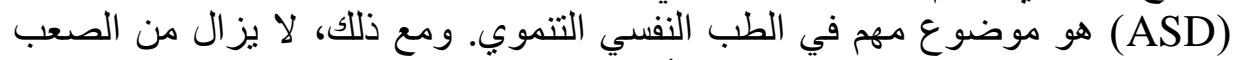

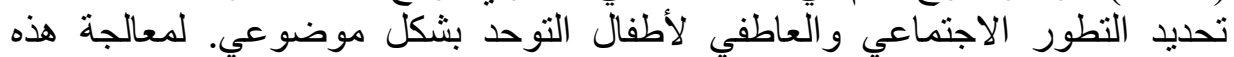

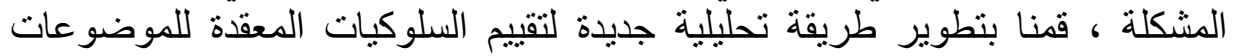

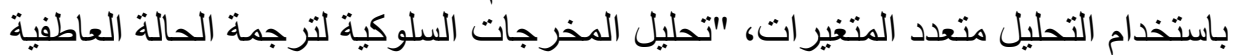
الكمية" (BOUQUET)

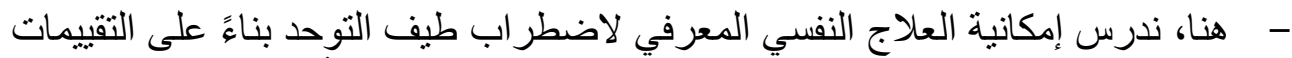

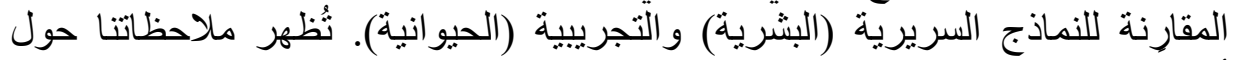
أطفال ASD (مقابل إخوتهم الذين يتطورونة بشكل طبيعي) والفرخ النية ) المنزلي في نماذج 
الحرمان الاجتماعي والحسي أهمية التحفيز الحسي أحادي الوسائط، وهو مهم بشكل

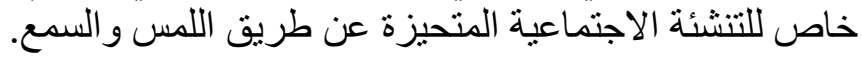

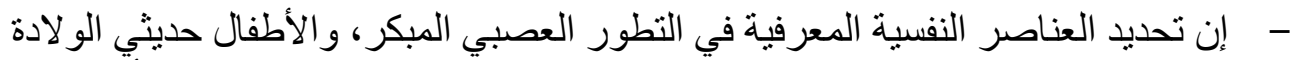

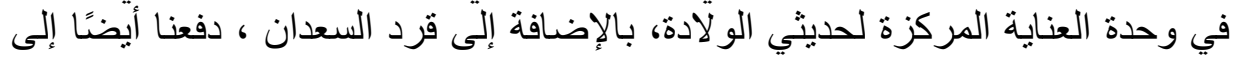

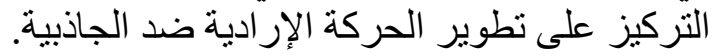

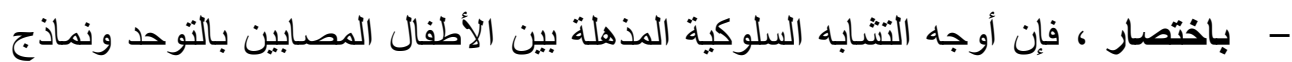

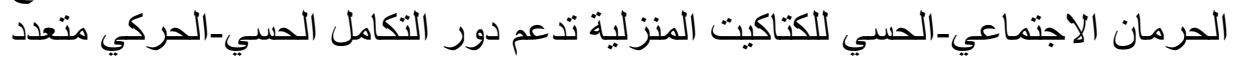

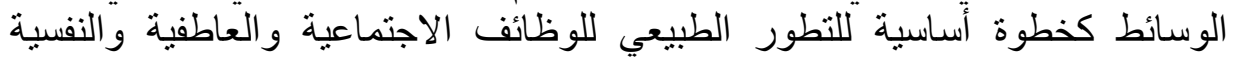

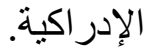

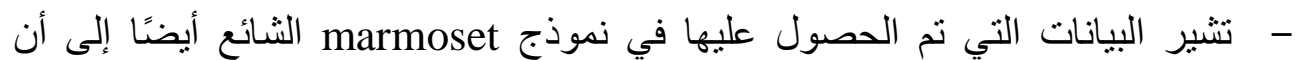

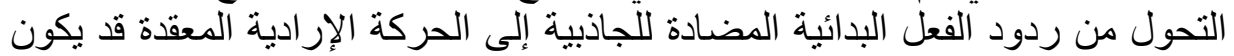

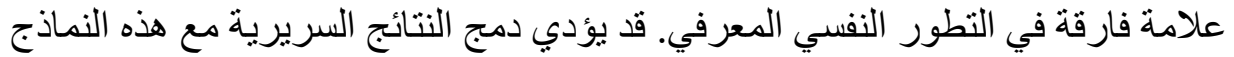

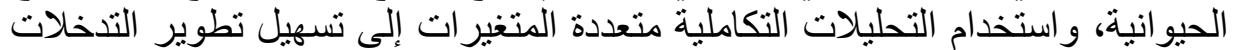

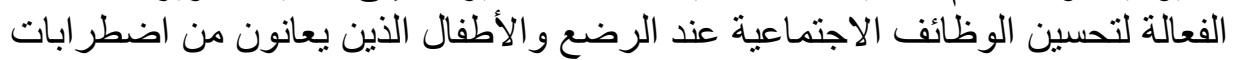

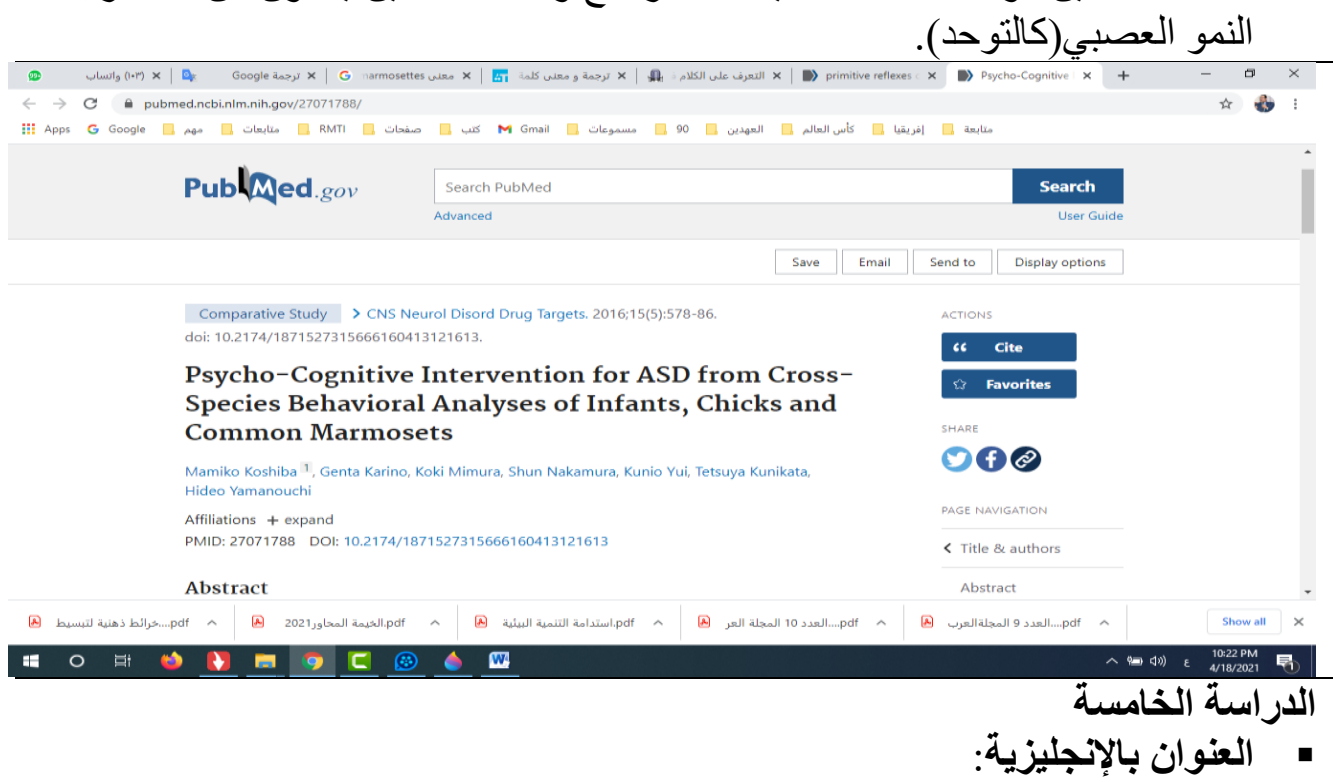

Evaluation of the effectiveness of MNRI therapy (Masgutova neurosensorimotor reflex integration) in children with autism 
based on the ATEC (autism treatment evaluation checklist) test - a $(\uparrow \cdot \backslash \wedge)$ preliminary report

$$
\text { الر ابط هو : }
$$

https://www.researchgate.net/publication/341402824 Evaluation of the Therapeutic Effect of MNRI_Reflex_Neuromodulation o n_Children Diagnosed with Autism Based on Reflex_Assessme nts QEEG Analysis and ATEC Questionnaire

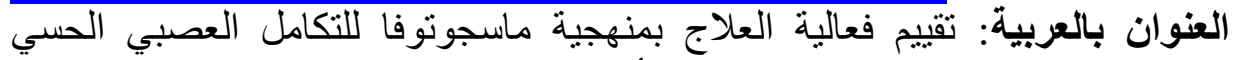

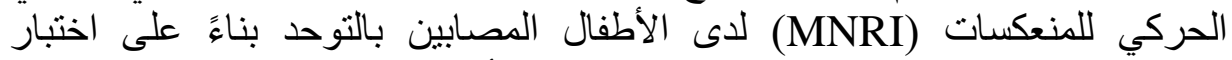
ATEC

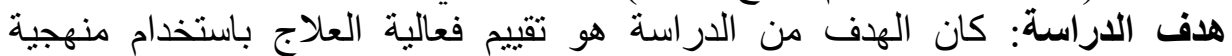

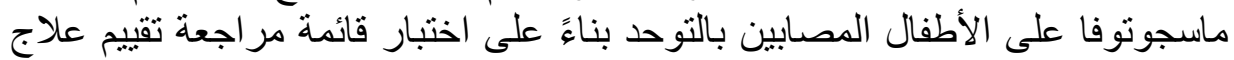
التوحد" (ATEC)

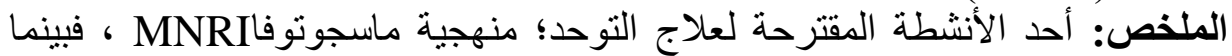

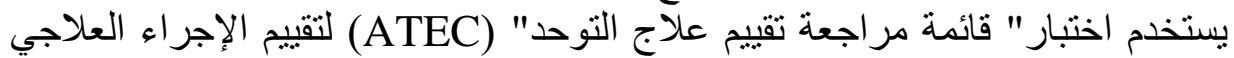

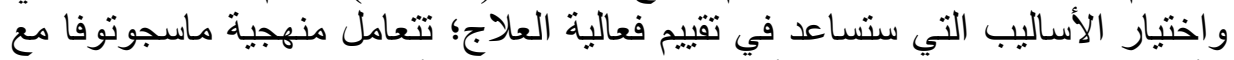

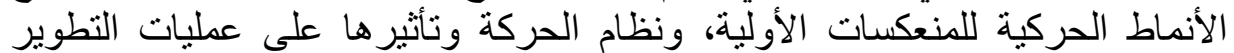

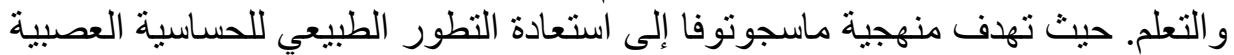

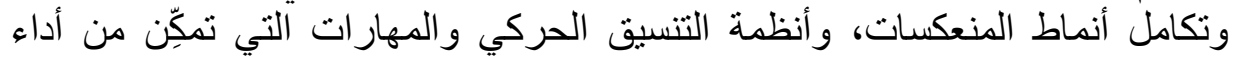
الحركة و التنظيم السلوكي و الوظائف العاطفية و المعرفية التهية.

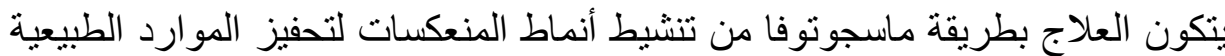

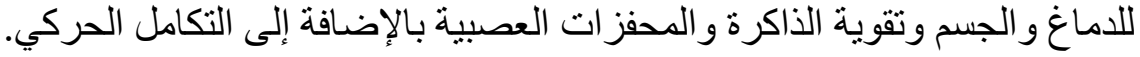

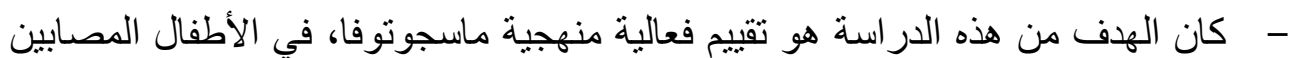

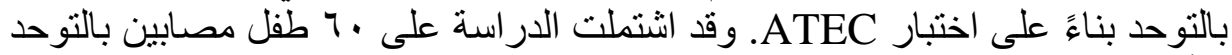

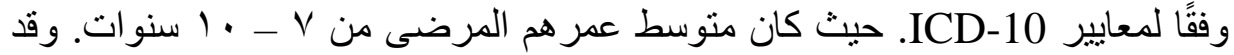

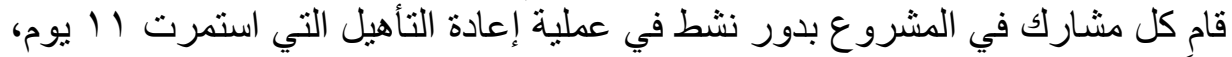

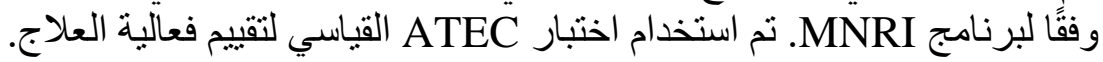

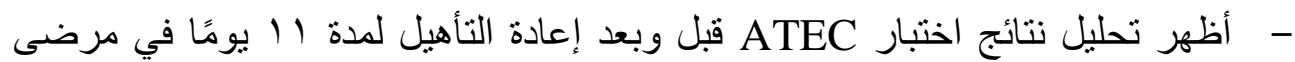

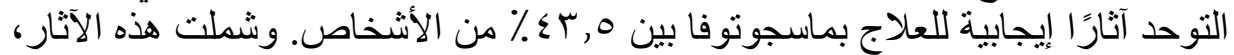

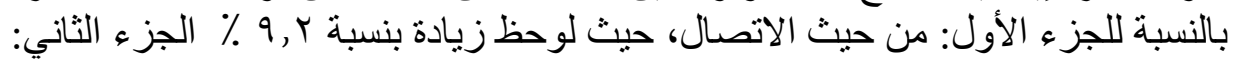

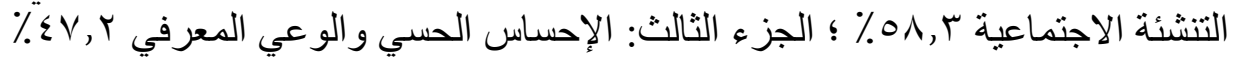

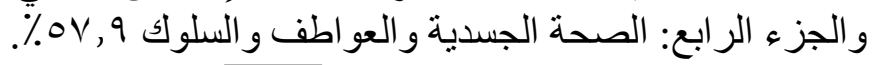




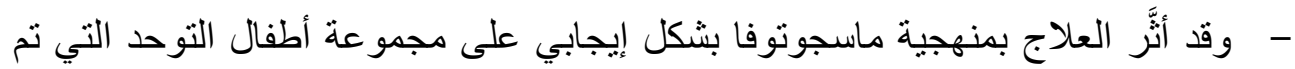

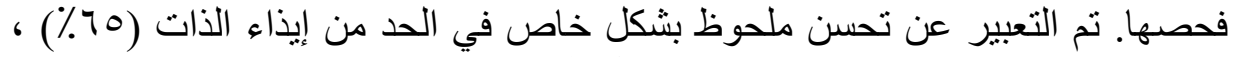

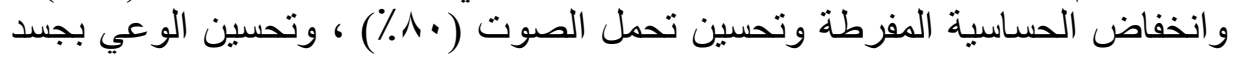

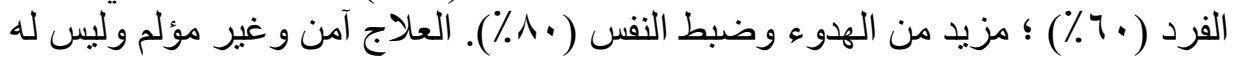

عنوان الدراسة: علة Evaluation of the Therapeutic Effect of MNRI Reflex neuromodulation on Children Diagnosed with Autism Based on

Reflex Assessments, QEEG Analysis and ATEC Questionnaire ترجمة العنوان: تقييم التأثير العلاجي لمنهجية ماسجوتوفا للتكامل العصبي الحسي الحركي للمنعكسات MNRI على الأطفال المصابين بالتوحد بناء على تقييم المنعكسات

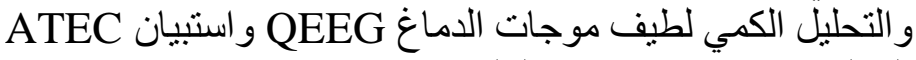
المؤلفون وتخصصاتهم العلمية: Katarzyna Novak, Piotr Sobaniec

Wojciech Sobaniec , Nelli Akhmatova, and Patty Shackleford

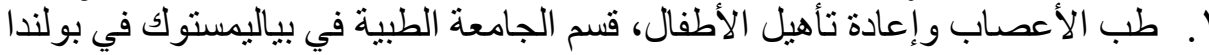

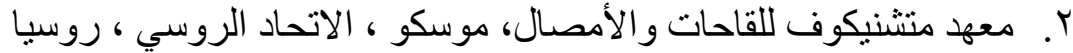

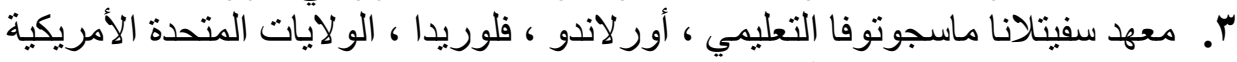

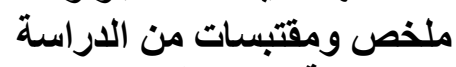
• مقدمة: لا يز ال بتعين اكتشاف فرضية التيات الأسباب النمائية العصبية لاضطراب طيف

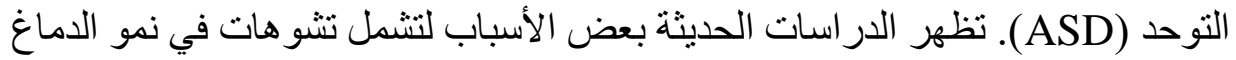
و الجهاز العصبي المركزي.

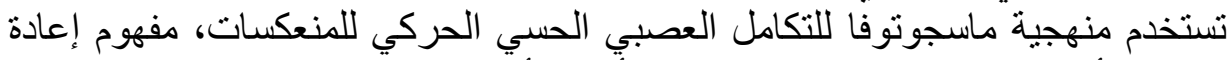

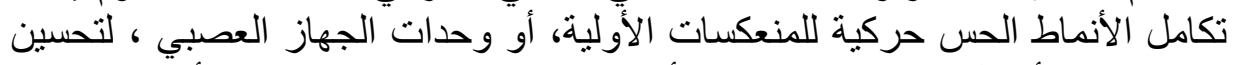

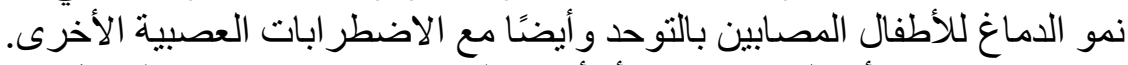

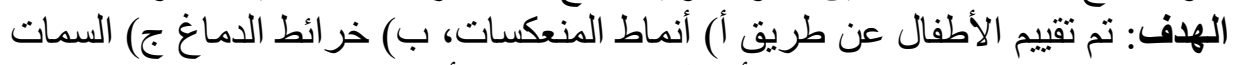

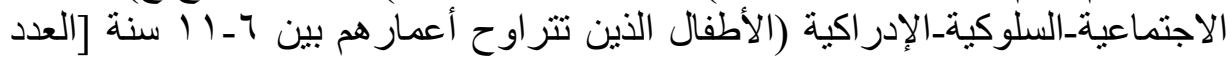

الطرق: طرق تقييم أ) التقييم العصبي الحسي الحركي للمنعكسات ، ب) التحليل الكمي

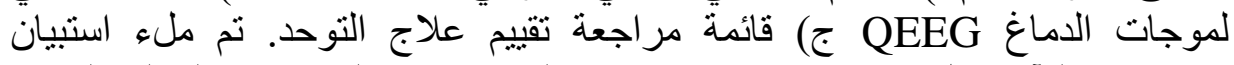
للآَاء قبل وبعد برنامج ماسجوتوفا، المستخدم في المؤتمر ات العة العائلية المكثفة 
النتائج: أظهرت جميع طرق التقييم الثلاثة تغير ات إيجابية كبيرة في الأطفال المصابين

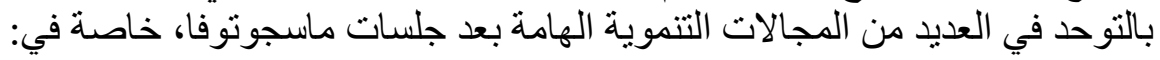

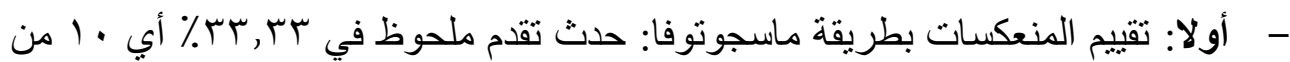

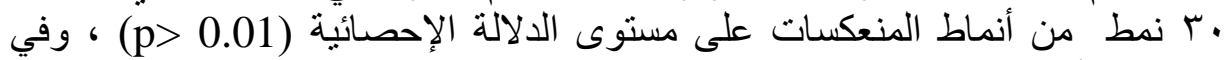

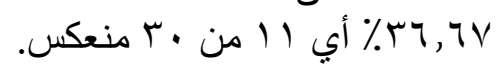

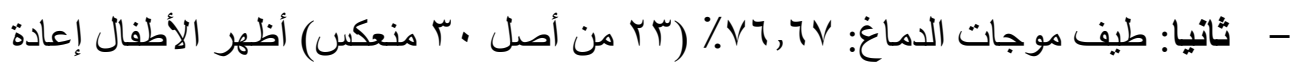

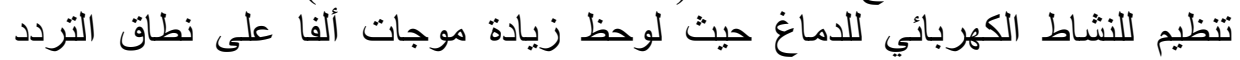

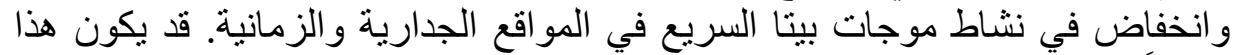

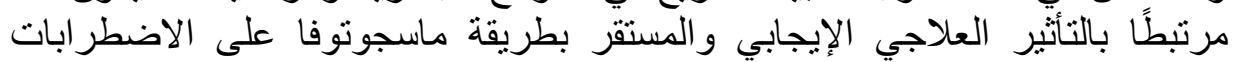
الحركية التي تنشأ في الجهاز العصبي الإبي المركزي.

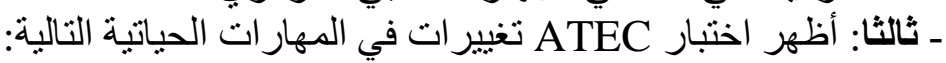

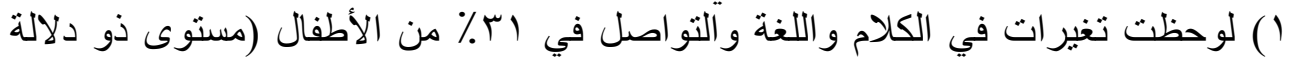

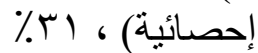

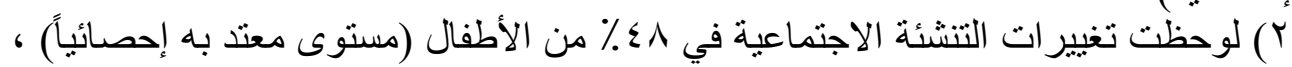

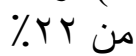
ץ) الإحساس الحسي و التغيرات في الوعي المعرفي لوحظت في ^ץ٪ من المشاركين

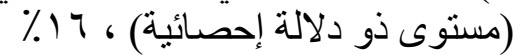

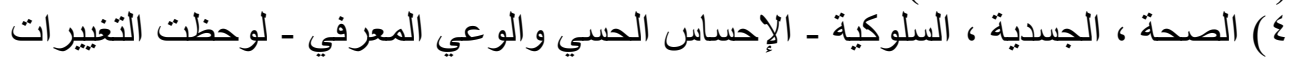

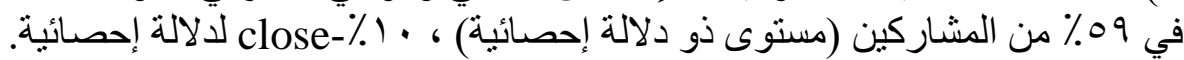

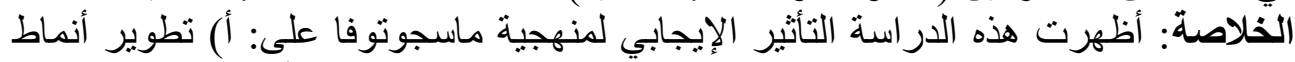

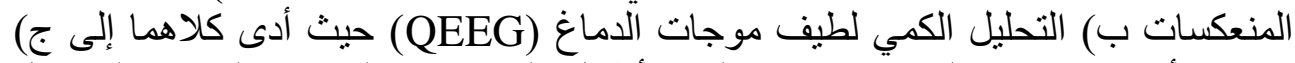

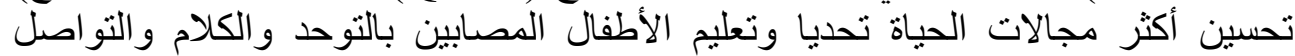

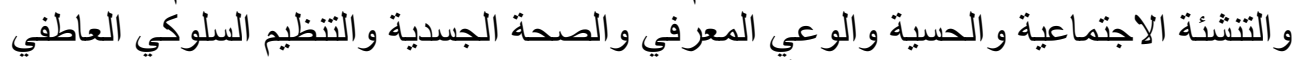

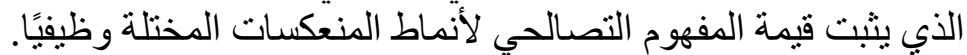

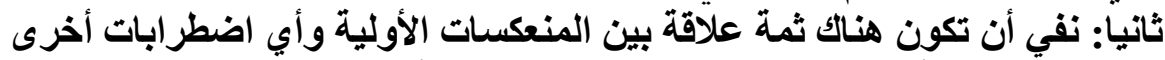

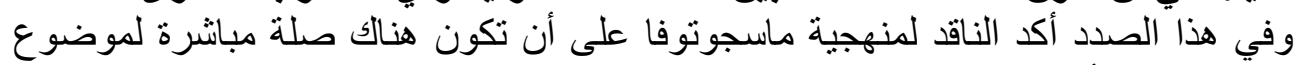

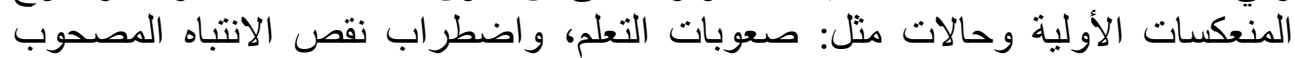

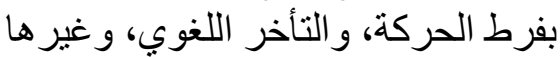

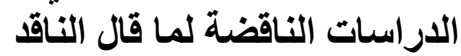
الاراسة الأولى عن علاقة المنعكسات الأولية بالتأخر اللغوات النوي

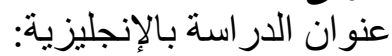




\section{Developmental Language Disorder and Uninhibited Primitive Reflexes in Young Children} • العنوان بالعربية: اضطراب اللغة النمائي و المنعكسات الأولية غير مكتملة النضج

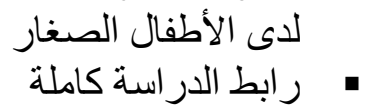

https://pubs.asha.org/doi/full/10.1044/2020_JSLHR-19-00423

• Maria Matuszkiewicz, Tadeusz Gałkowski المؤلفون

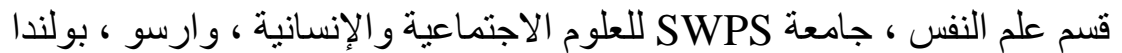

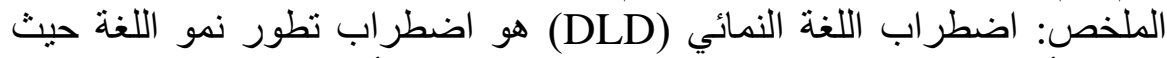

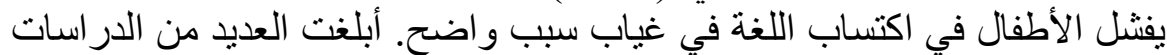

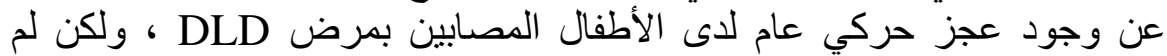

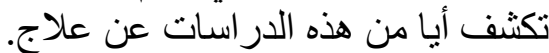

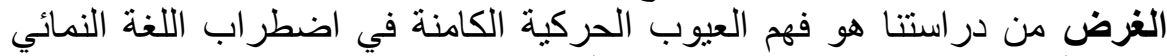

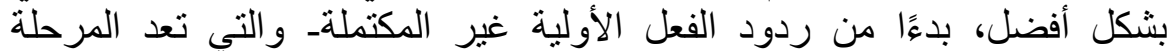

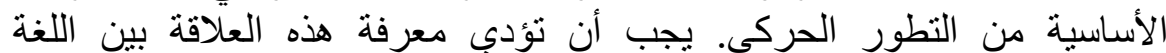

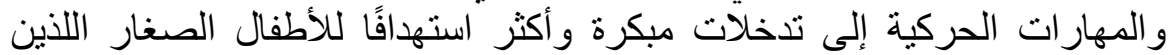

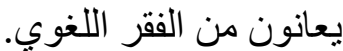

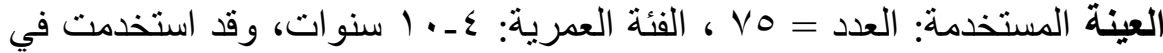

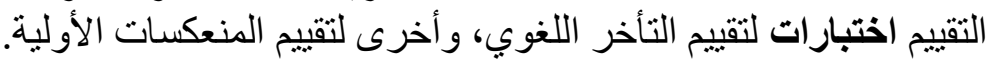

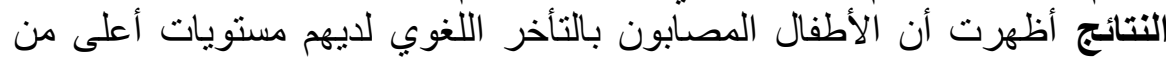

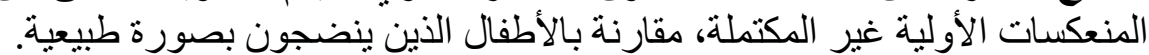

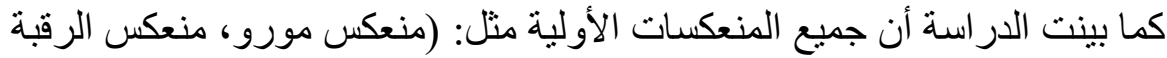

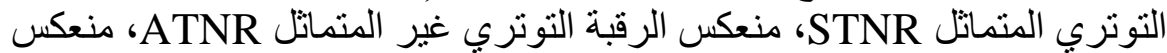

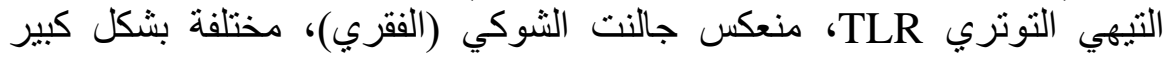

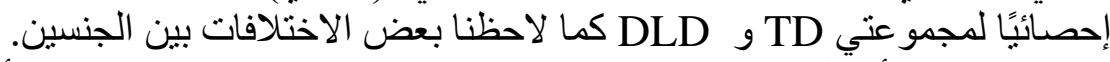

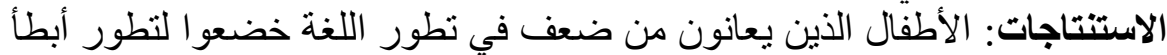

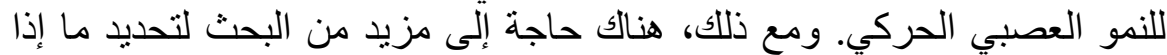

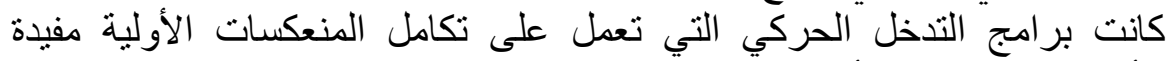
للأطفال المصابين بالتأخر اللغوي. 


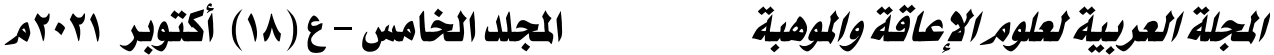

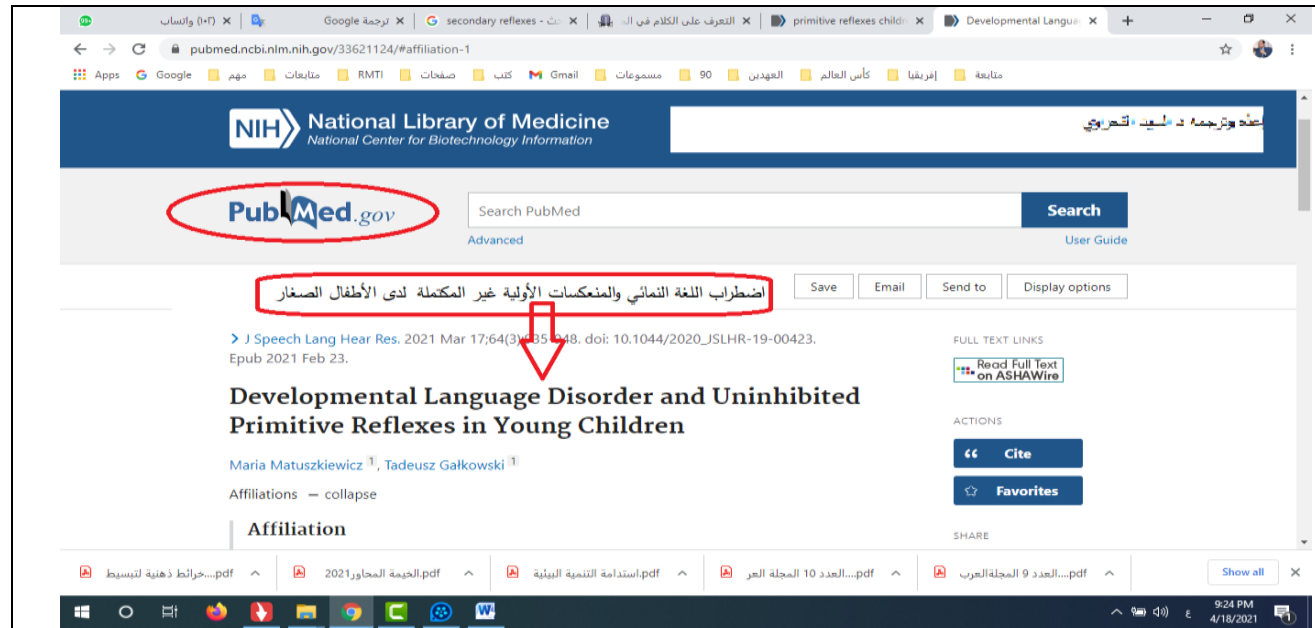

الدراسة الثانية: عن علاقة المنعكسات الأولية باضطراب فرط الحركة ونقص الانتباه

Persistent Childhood Primitive Reflex عنوان -

Reduction Effects on Cognitive, Sensorimotor, and Academic Performance in ADHD

ترجمة العنوان: التأثثر السلبي للمنعكسات الأولية غير مكتملة النضج (المستمرة)

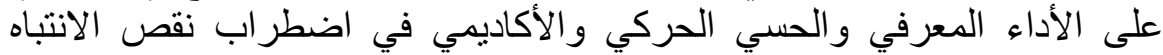

$$
\text { رفرط الحركة الدراسة }
$$

https://www.frontiersin.org/articles/10.3389/fpubh.2020.431835/full?\&utm source=Email_to_authors_\&utm_medium=Email\&utm_content=T1_11.5e1 _author\&utm_campaign=Email_publication\&field\&journalName=Frontiers in_Public_Health\&id=431835\&fbclid=IwAR1E5Xa6uEfnhLmFZ0WPp9g meb-GA2Tjatdk0edSbUXsDItayr4X6GelMH4

$$
\text { - أبرز النتائج }
$$

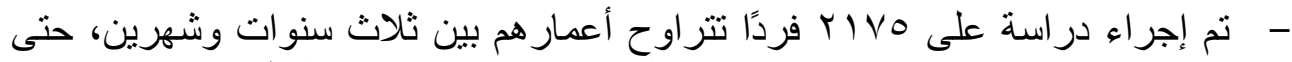

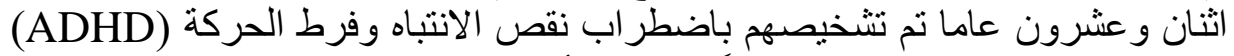

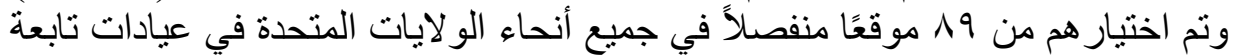

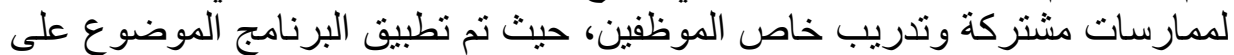

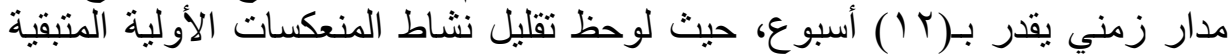

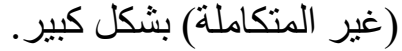


- كان الهدف هو تحديد مدى فعالية برنامج تدريبي قائم على تفعيل نصفي المخ الكروي،

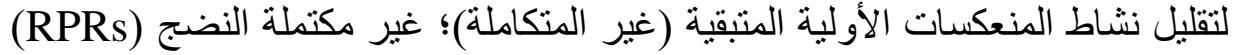

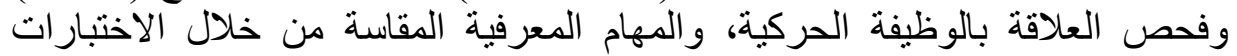

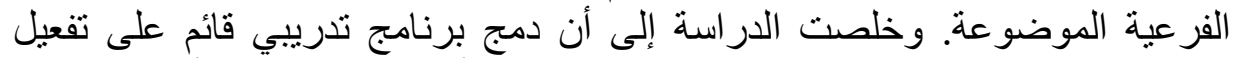

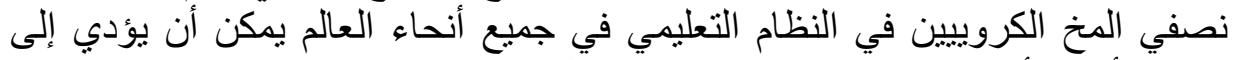
زيادة الأداء الأكاديمي و المعرفي و الحركي بشكل غير مكلف نسبيًا.

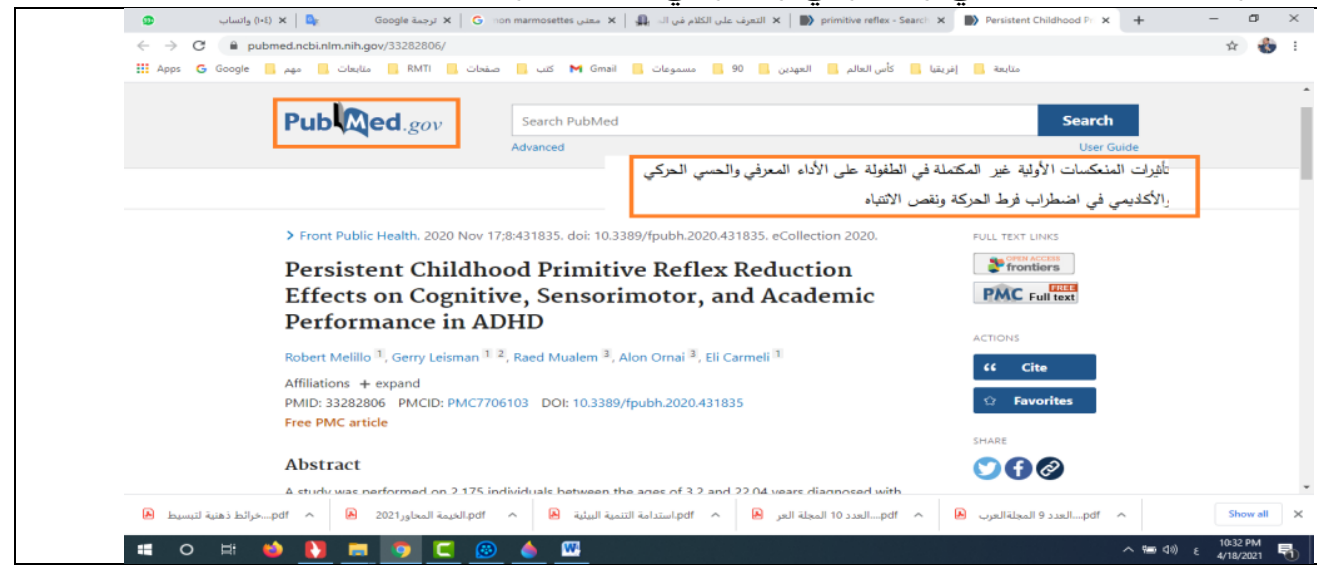
الاراسة الثالثة: عن علاقة المنعكسات الأولية بالمهارات المعرفية

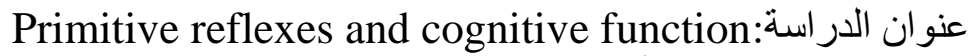
الترجمة: المنعكسات الأولية و الوظيفة المعرفية رابط الدر اسة:

https://www.scielo.br/j/anp/a/ZYT9THL7vPQdd5t7S9nn33c/abstr act/?lang=en

Alfredo Damasceno , Adriane M ) المؤلفون هم سبع علماء برازيليون وهم Delicio, Daniel F C Mazo, João F D Zullo, Patricia Scherer, Ronny (T Y Ng, Benito P Damasceno جهات العمل والتخصص: قسم طب الأعصاب، كلية الطب، جامعة ولاية كامبيناس، كامبيناس، البرازيل. الهذف: دراسة العلاقة بين المنعكسات الأولية (PR) في مرحلة البلوغ، وأهميتها

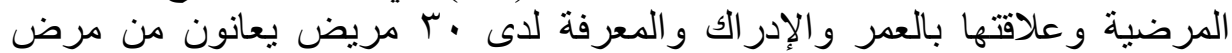
الز هايمر المحتمل (AD) و ع و 1 شخصاً ضالابطاً. 
• الطريقة: استتد تشخيص مرض الزهايمر المحتمل إلى معايير DSM-IV و

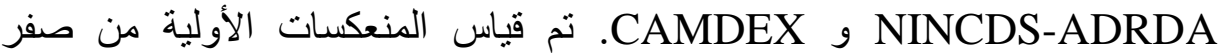

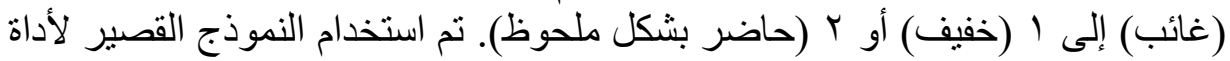

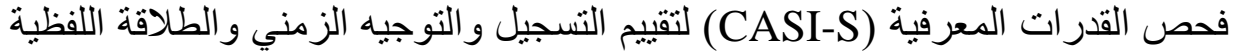

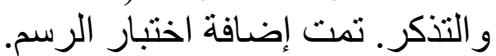

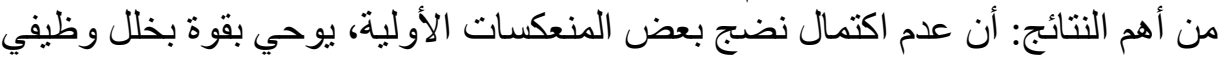

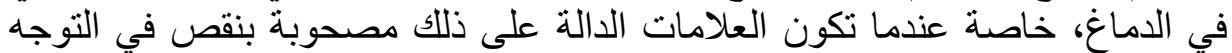

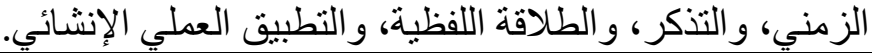

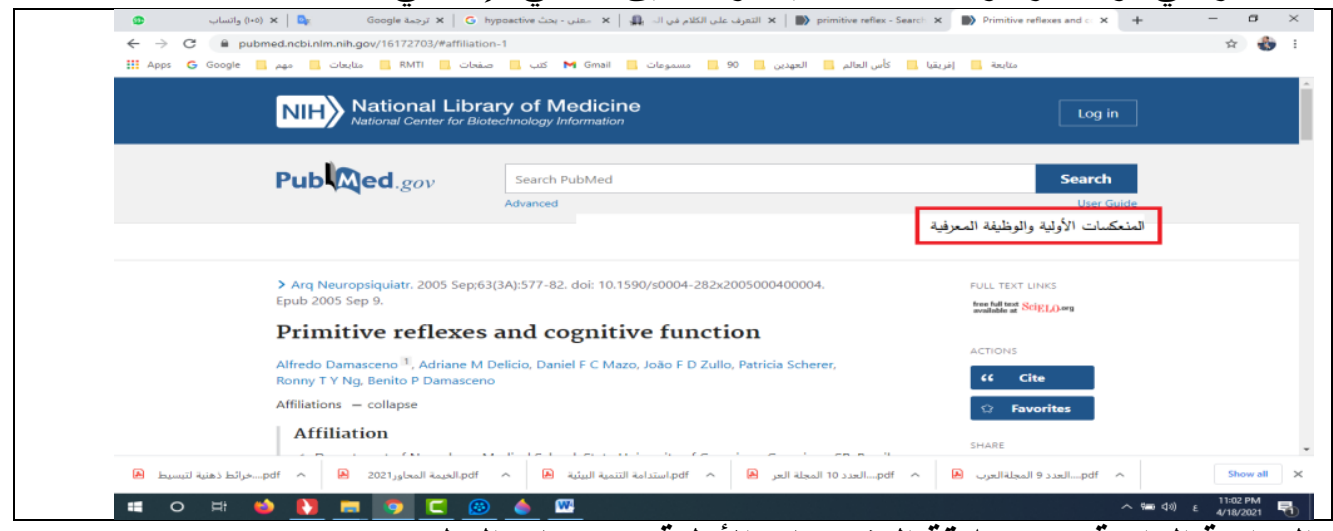
الاراسة الرابعة: عن علاقة المنعكسات الأولية بصعوبات التعلم

عigns of neurobehavioral dysfunction in a sample of learning disabled children: stability and concurrent validity الترجمة: علامات الخلل السلوكي العصبي في عينة من الأطفال ذوي صعوبات التعلم:

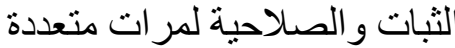
رابط الدراسة

https://journals.sagepub.com/doi/abs/10.2466/pms.1985.61.3.863 -

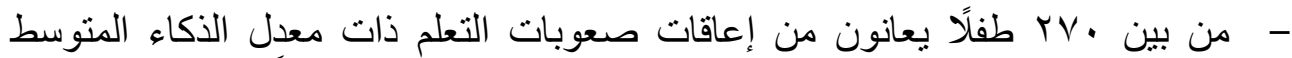

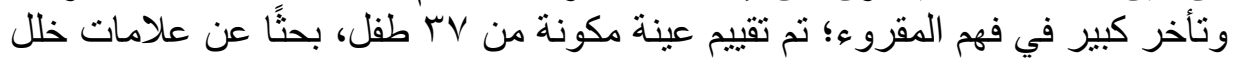
في السلوك العصبي. - - حيث تم تقييم كل هذه العلامات (التي كان أولها): المنعكسات الأولية، وتفاعلات

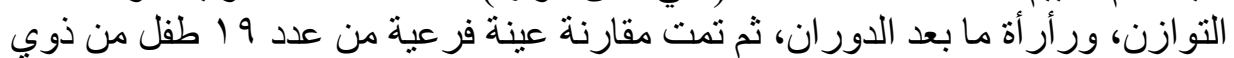


الإعاقة العقلية مع عينات من أطفال عاديين وطبيعين من الناحية التطورية لمنعكس

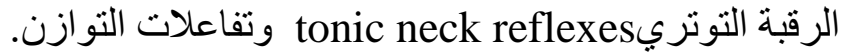

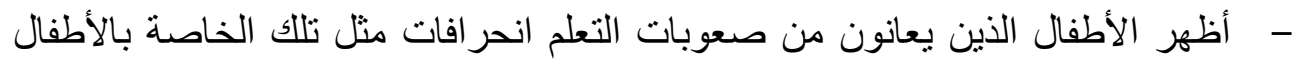

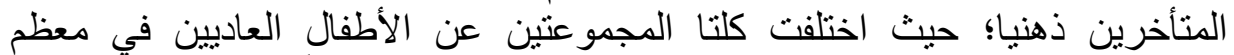

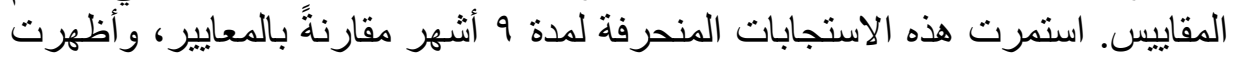

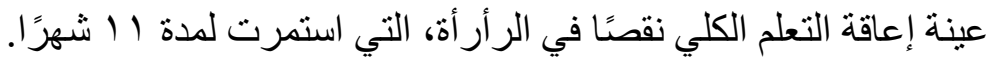

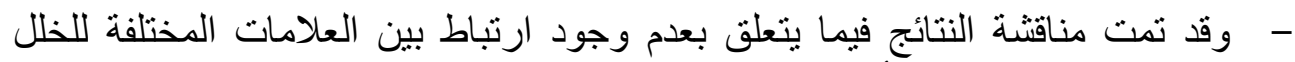

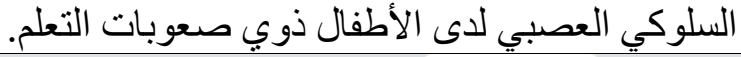

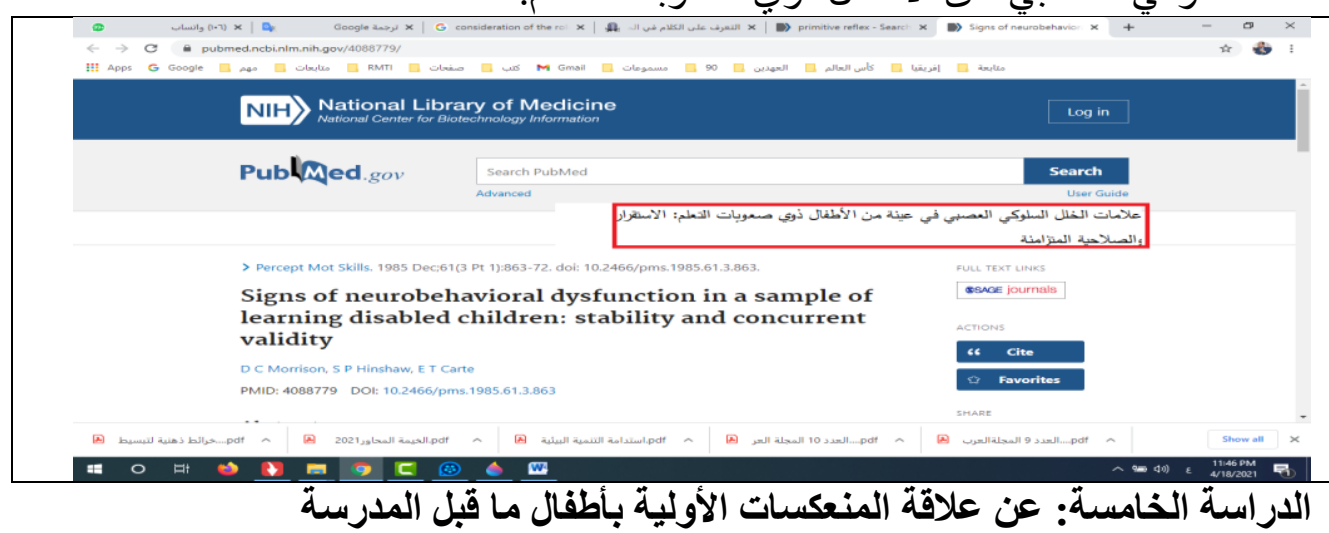

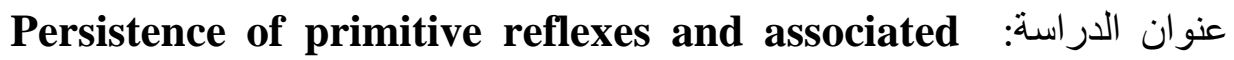
motor problems in healthy preschool children الترجمة: المنعكسات الأولية غير المكتملة والمشاكل الحركية المرتبطة بها لدى أطفال ما قبل المدرسة الأصحاء الأعكات رابط الدر اسنة المدرسة

https://www.ncbi.nlm.nih.gov/pmc/articles/PMC5778413/ المؤلفون: Anna M Choińska , Małgorzata

Paprocka-Borowicz التخصصات العلمية و العمل ا. مختبر تطوير إعادة التأهيل، قسم العلاج الطبيعي، كلية العلوم الصحية، جامعة

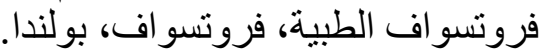
r. قسم العلاج الطبيعي، كلية العلوم الصحية، الجامعة الطبية في فروتنسواف، فرواف فروتسو اف، بولندا.

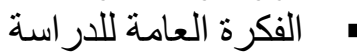




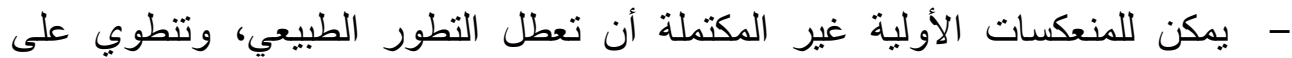

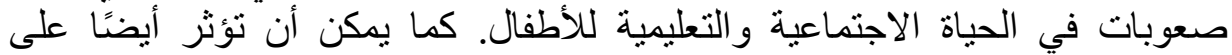

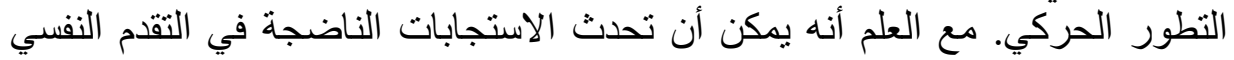

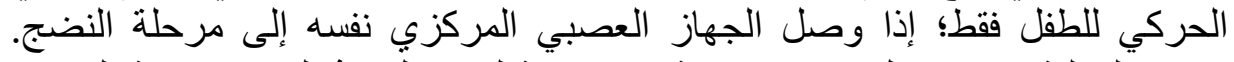
تتكون العملية من الانتقال من الاستجابة الانعكاسية لجذع الدئ الدماغ إلى الدى الاستجابة التي يتم التحكم فيها في القترة المخية التفال - - تحدد هذه الدراسة عدد من المنعكسات الأولية لدى الأطفال الأصحاء الذين تتراوح

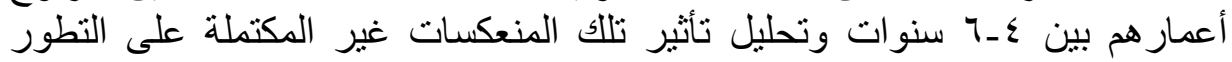

$$
\text { • النفسي الحركي. }
$$

- - يفضل إدخال علاج المنعكسات غير مكتملة النضج لدى الأطفال ذوو المهارات النفسية

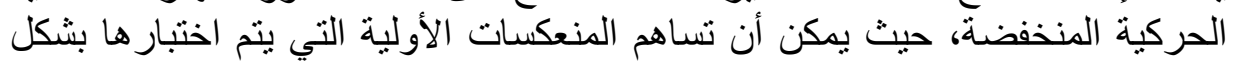

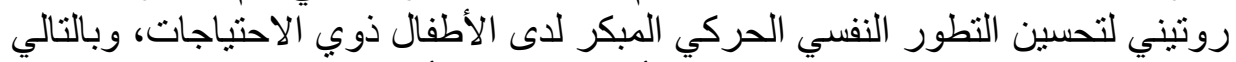

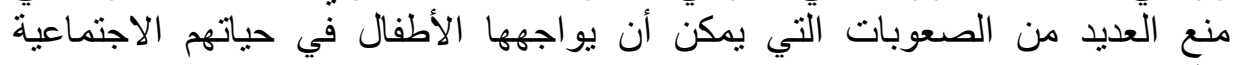

$$
\text { • ب المدرسية. }
$$

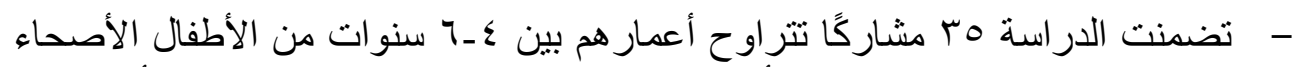

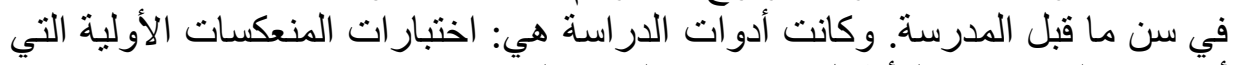

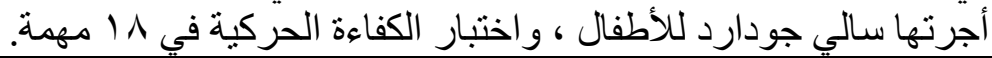

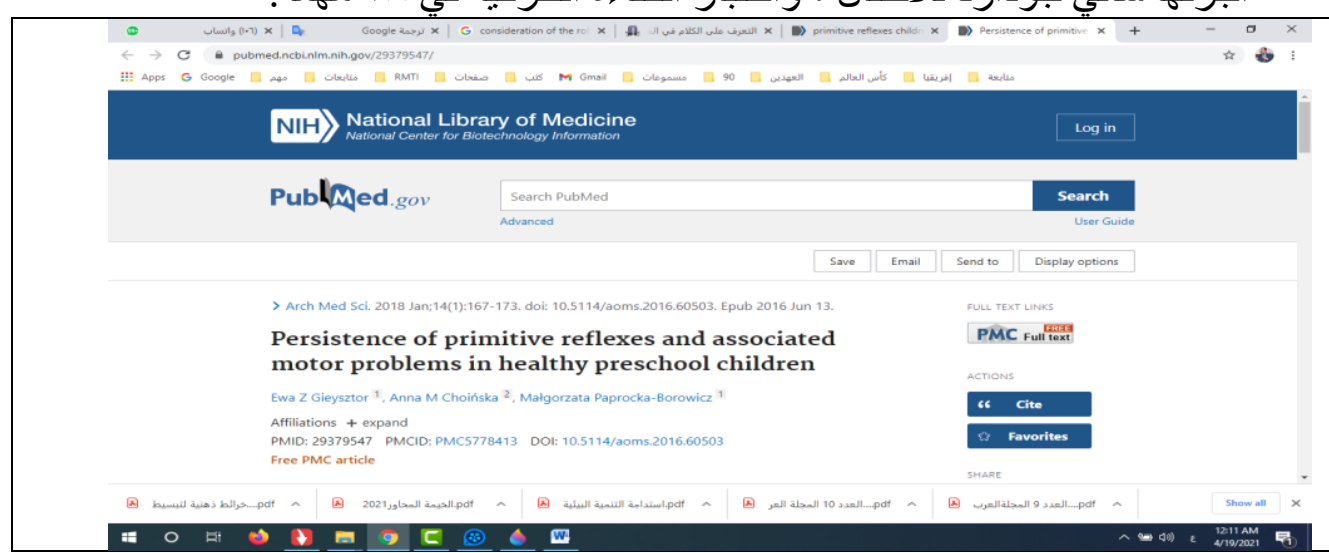


الثبهة الثالثة: الإدعاء بأن منهجية ماسجوتوفا غير معترف بها الإنها

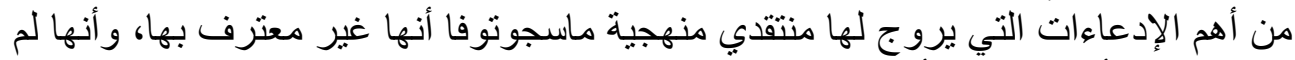

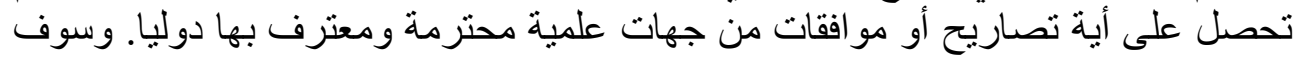

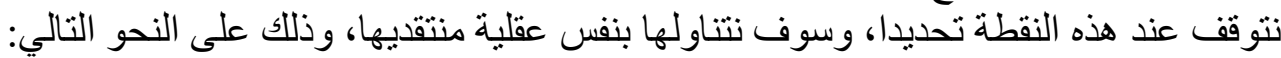

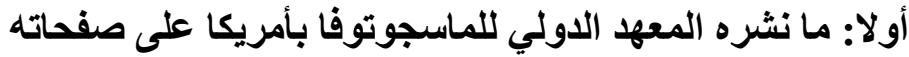

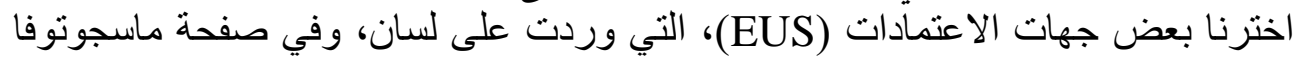

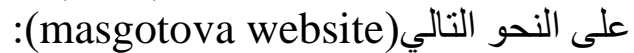

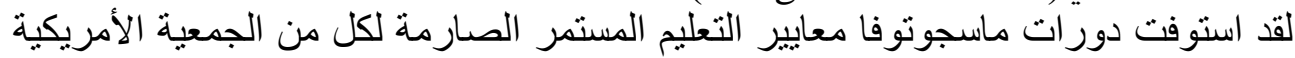

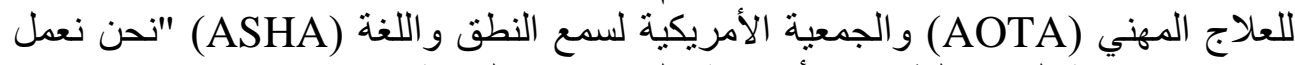

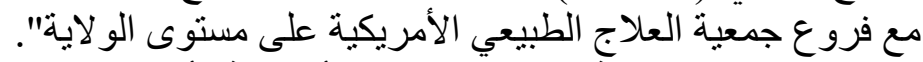

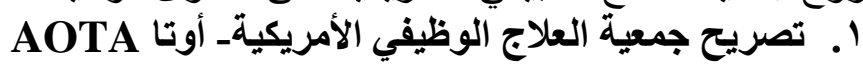

American Occupational Therapy Association يتمثل أحد الأهداف الأساسية لجمعية العلاج الوظيفي الأمريكية في تعزيز المعايير المهنية

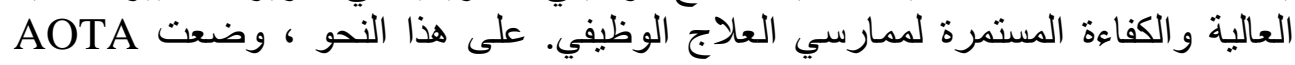

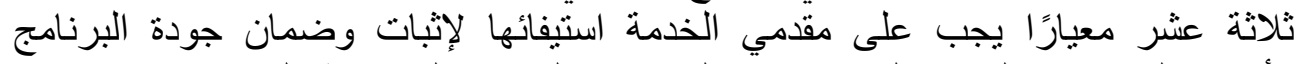

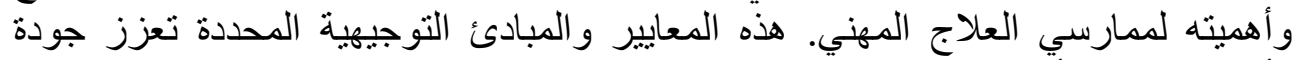

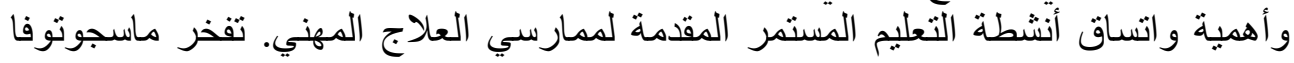

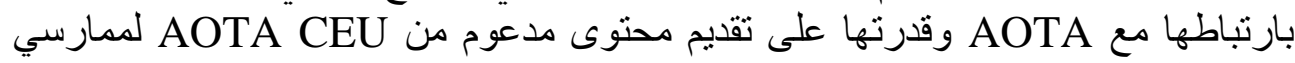
العلاج الوظيفي.

Y. . موافقة وتصريح الرابطة الأمريكية للسمع والنطق واللغةـ الآثـا American Speech-Language-Hearing Association-ASHA

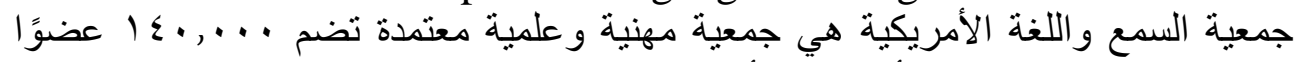

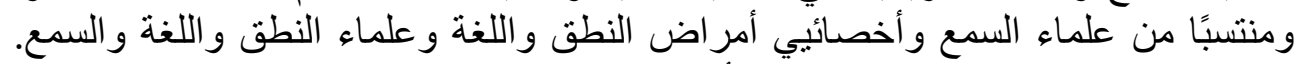

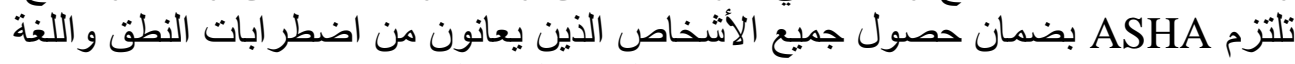

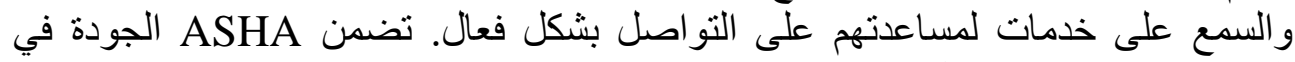

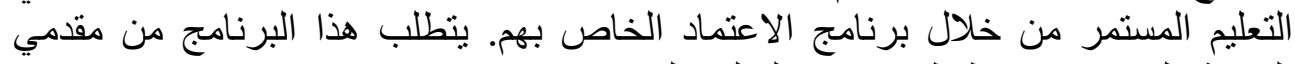

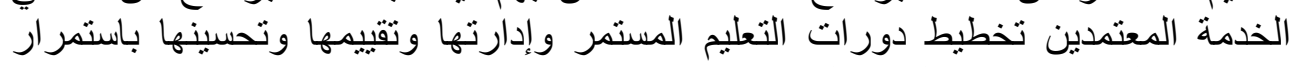

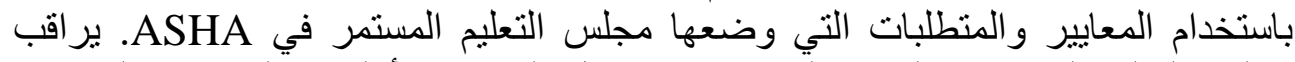

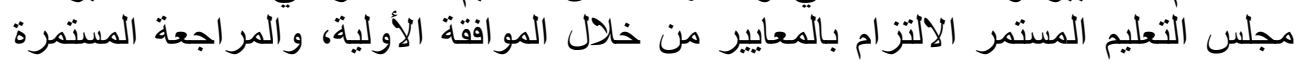

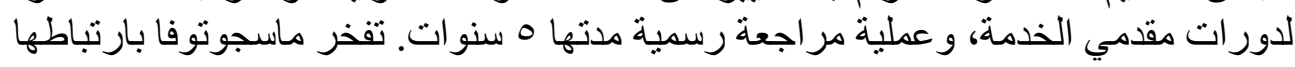
مع ASHA وقدرتها على تقديم دورات CEU المعتمدة من واللغة و السمع. 


\section{r. جمعية العلاج الطبيعي الأمريكية ـ أبتا}

American Physical Therapy Association -APTA

جمعية العلاج الطبيعي الأمريكية (APTA) هي منظمة مهنية وطنية تمثل ل.......

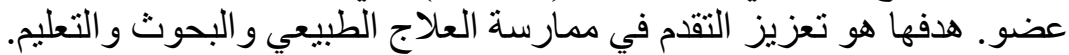

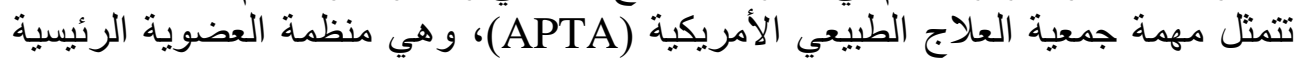

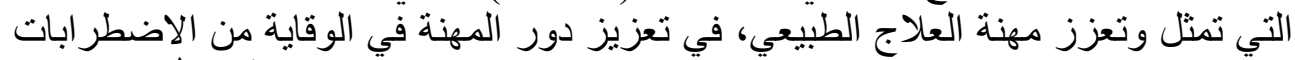

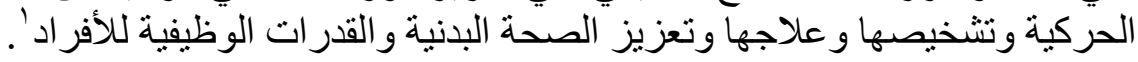

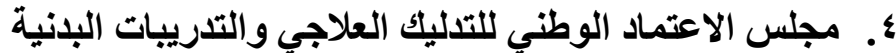

National Certification Board for Therapeutic Massage \& -NCBTMB

Bodywork

يعد مجلس الاعتماد الوطني للتدليك العلاجي و التدريبات البدنية (NCBTMB) منظمة

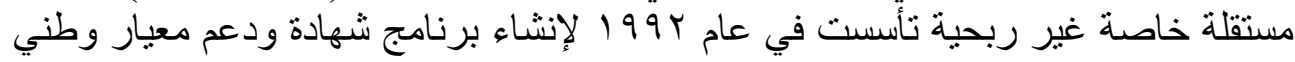

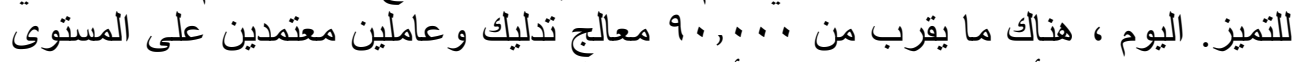

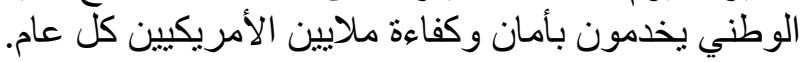

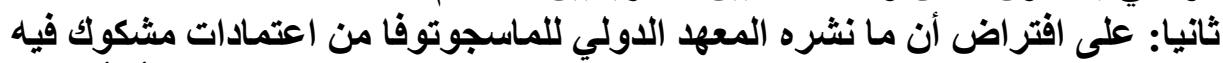

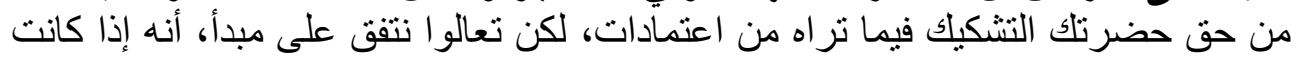

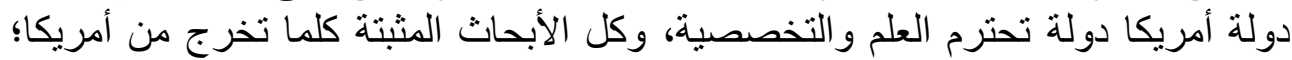

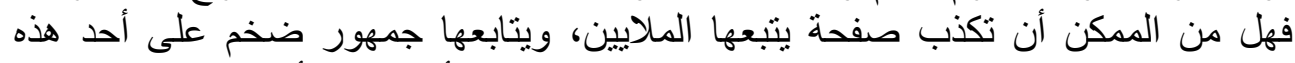

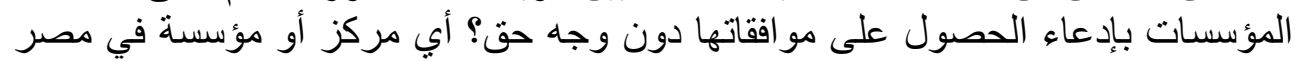

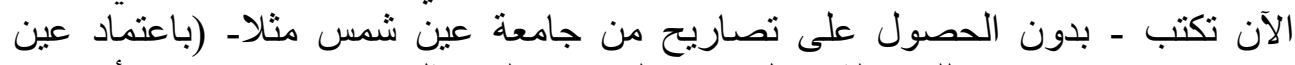

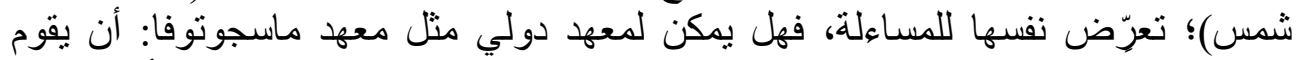

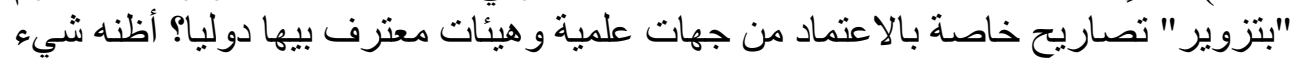

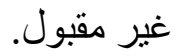

ثالثا: شخصية الاكتورة ماسجوتوفا نفسها

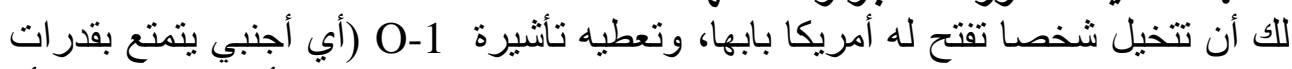

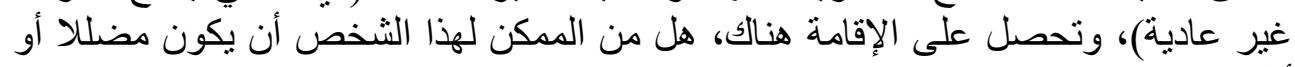

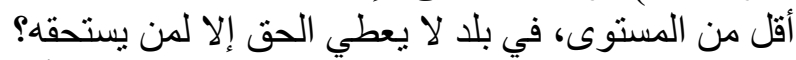

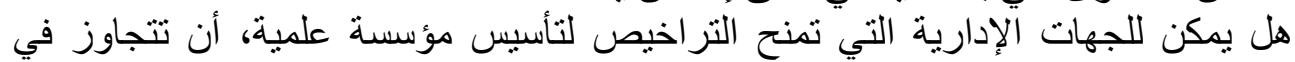

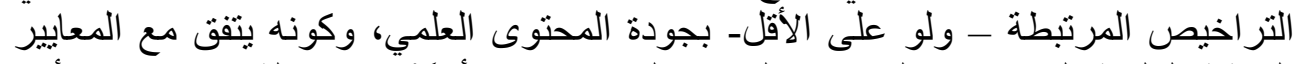

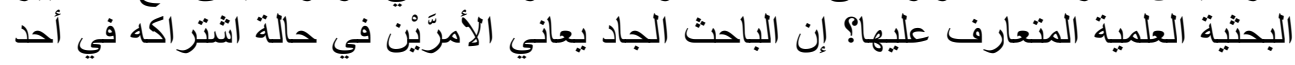

${ }^{1}$ - https://www.linkedin.com/company/american-physical-therapy-association/ 71 


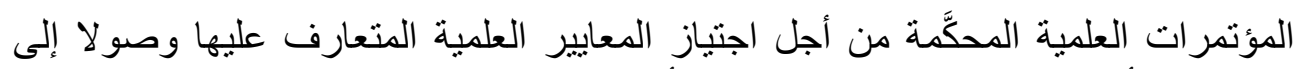

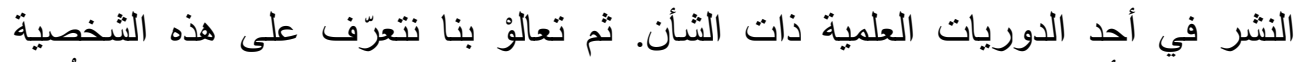

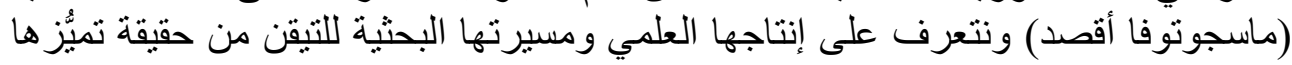
ونفي القدح فيها:

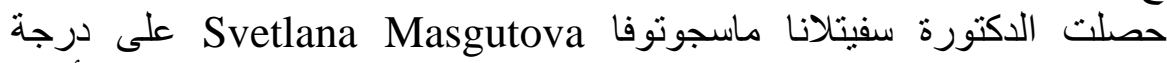

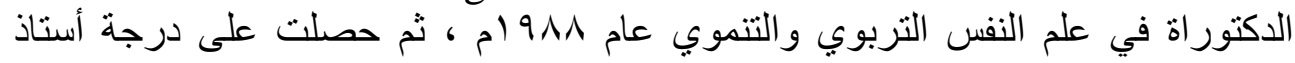

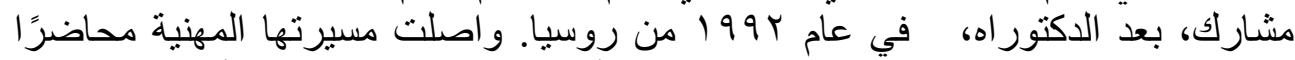
بالجامعة وعميدًا لقسم علم النفس التطبيقي، وأيضًا عملت كباء واحثة في في أكاديمية التعليم الرتمدتي. في أعمالها وأطروحتها للاكتوراه على أعمال علماء أمثال:إل فيجوتسكي

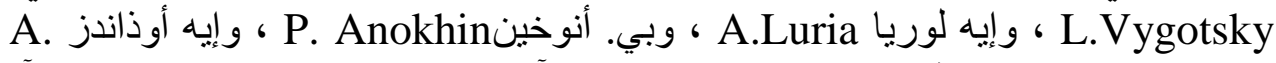
Uznadze

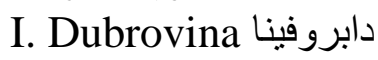

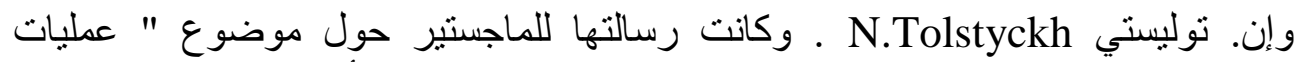

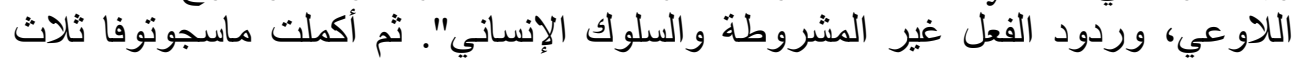

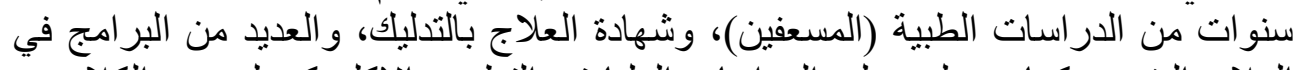

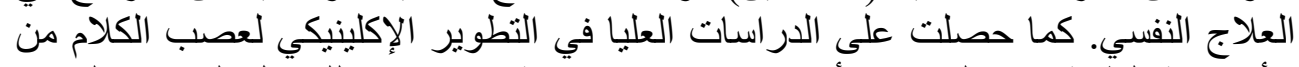

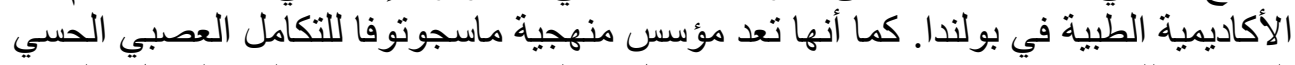

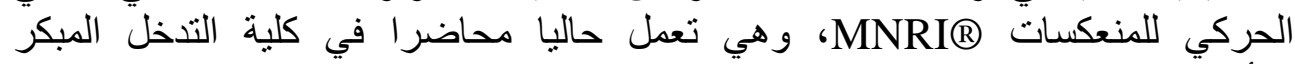

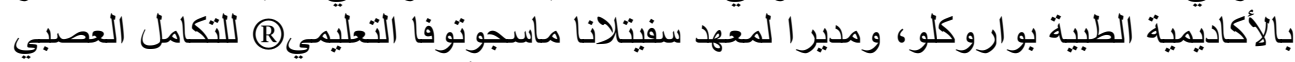

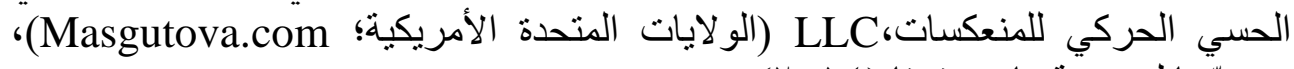

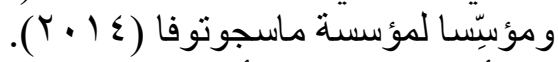

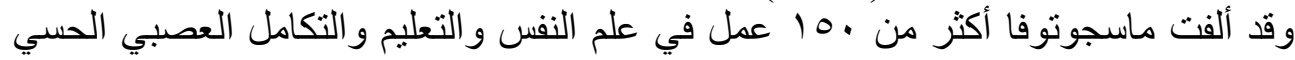

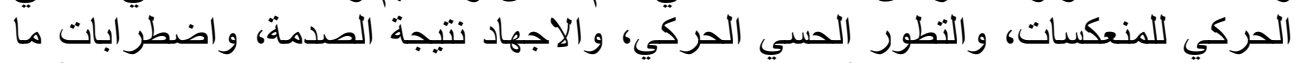

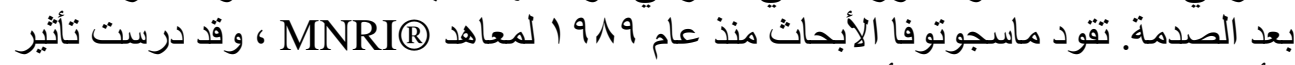

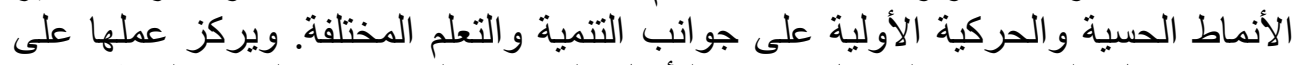

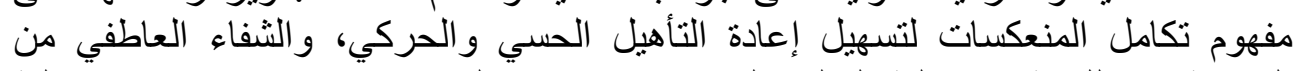

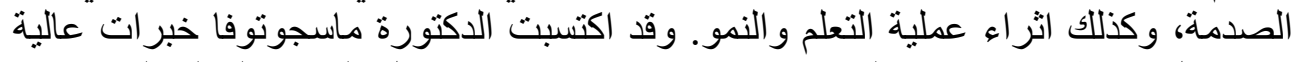

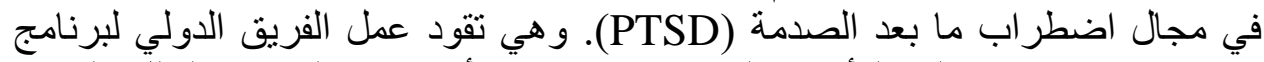

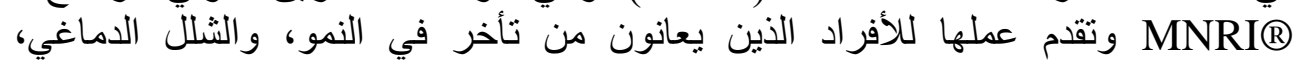

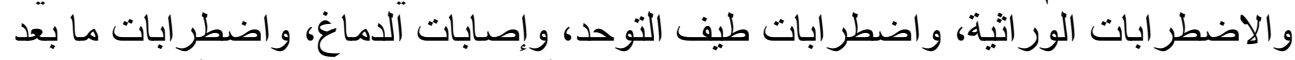

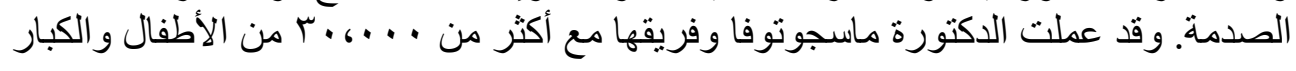




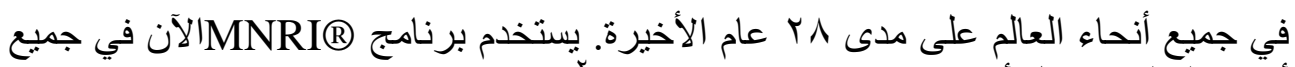

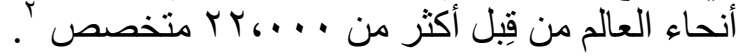

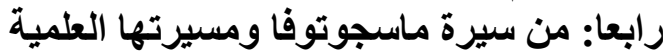

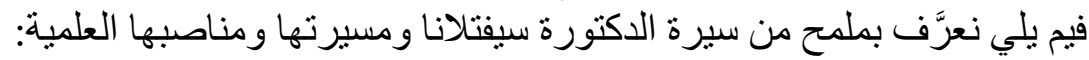

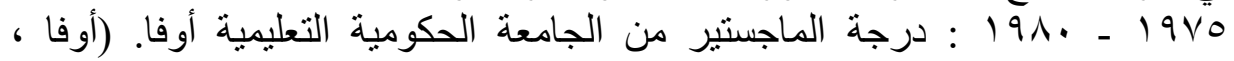

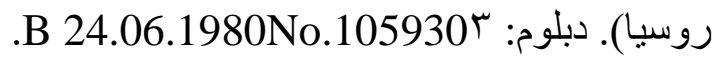
. 19 ـ ـ19 19 : مساعد مدرس، قسم علم النفس، الجامعة الحكومية التعليمبة أوفا. (أوفا، روسيا). ع 191 ـ 1911 : دراسات عليا، علم النفس العام والتربوي، معهد البحث العلمي التابع لأكاديمية التعليم الروسية، (موسكو). الدكتور اه "المشاكل النفسية الرئيسية للمر اهقين وبر امج المساعدة". دكتور اه، علم النفس التربوي

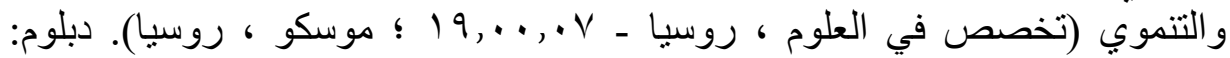

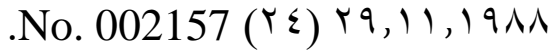

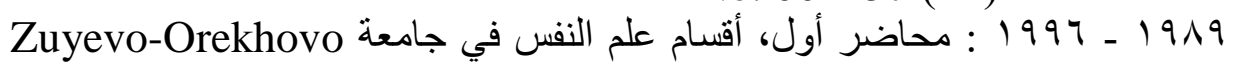
الحكومية التعليمية.

مؤسس و عميد الدر اسات العليا لعلم النفس العملي (منطقة موسكو ، روسيا).

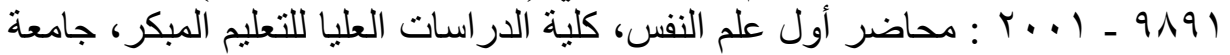

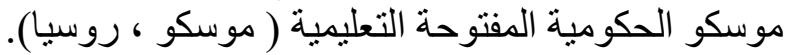

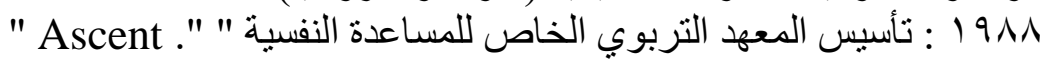

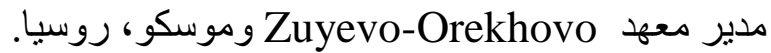

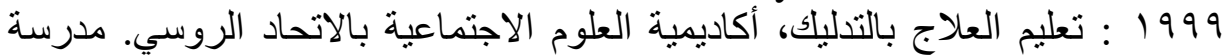

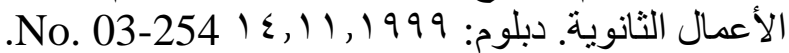

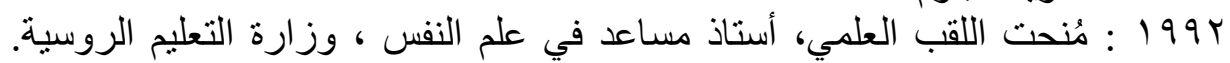
No. 17.09.1992 ‥r/r.

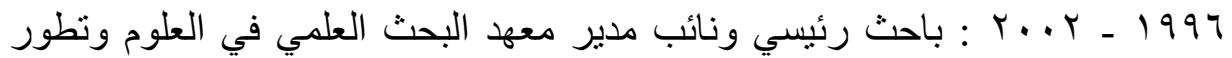

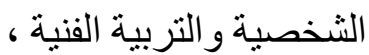
رئيس أكاديمية التربية الروسية ؛ ومختبر أبحاث الحركة وتطور الثخصية (موسكو).

${ }^{2}$ - https://masgutovamethod.com/about-the-svetlana-masgutova-educationalinstitute/svetlana-masgutova-ph-d 


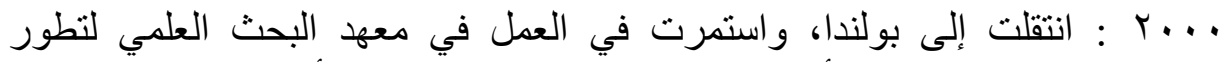
الثخصية والتربية الفنية، وأكاديمية التربية الروسية ؛ ومختبر أبحاث الحركة التهري وتطور الثخصية (موسكو).

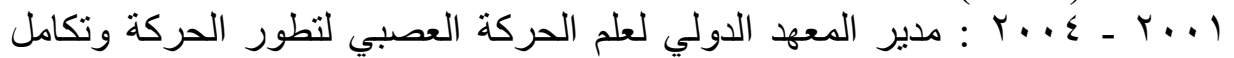

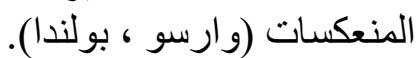

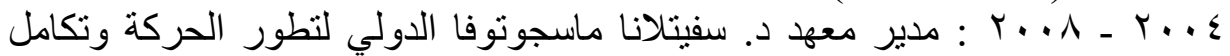

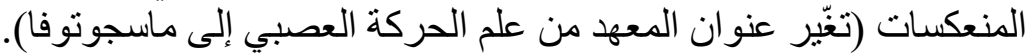

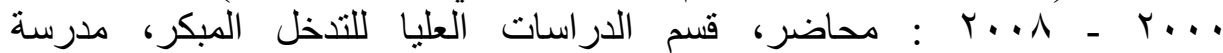
Dolnoslaski

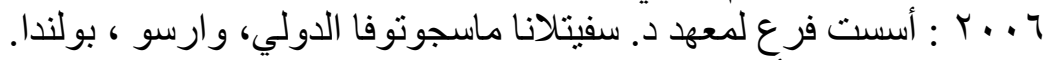

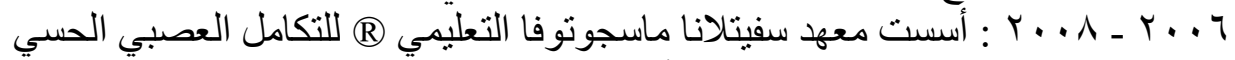

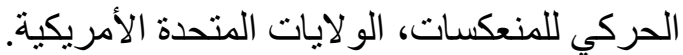

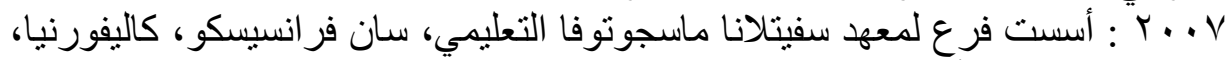

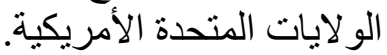
A . . ب : درجة الدراسات العليا، التدخل المبكر وتطوير الكلام العصبي، قسم الصحة

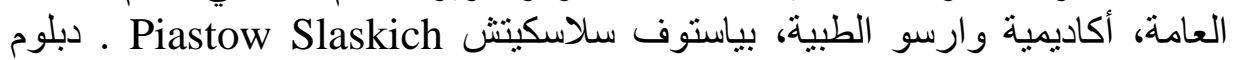

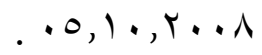

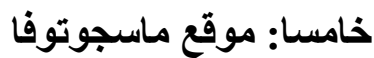

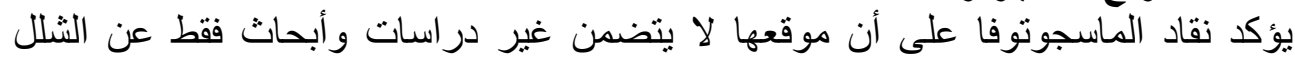

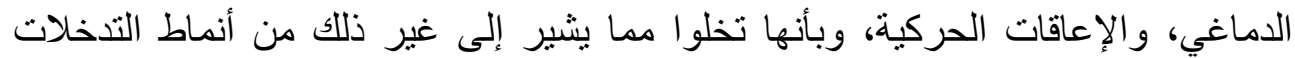

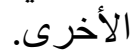
و أنا أدعو أب شخص، قادر على التعامل مع النت، ولديه حد أدني من المعرفة من الدخول https://masgutovamethod.com/ https://www.masgutovafoundation.org/ https://www.facebook.com/SvetlanaMasgutovaEducationalInstitute/ و التأكد من صحة أو زيف هذا الكلام الذي ذكر.

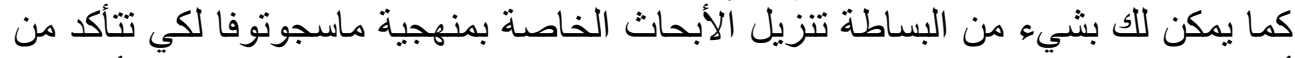

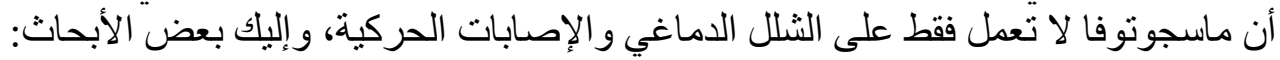


1. Masgutova, S., Akhmatova, N., Sadowska, L., Shackleford, P., \& Akhmatov, E. (2016). Progress with neurosensorimotor reflex integration for children with Autism Spectrum Disorder. Journal of neurology and psychology, 4(2), 14.

2. Masgutova, S., \& Sadowska, L. (2006). The Use of the Edu-K for Children with Learning Difficulties: Early Developmental Dynamic. In materials of: Polish National Conference: "NeuroKinesiology as the Method of Facilitation of Development and Learning for Children and Youth with Dyslexia, ADHD, Autism and Asperger (Vol. 8, No. 2006, pp. 39-50).

3. Masgutova, S. K. (2000). NeuroKinesiological basis of the reflex integration for successful learning of the child. In materials of Polish National Conference:"NeuroKinesiology as the Method of Facilitation of Development and Learning for Children and Youth with Dyslexia, ADHD, Autism and Asperger." 08.04. 2006.(P1.).

4. Masgutova, S. K. (2006). Neuro-Kinesiology as the support for development and learning. Children with Learning Challenges and ADHD. In Materials of Polish National Conference: "Modern Methods of Stimulation of Movement Development and Learning in Children with Difficulties in Learning, ADHD and Autism (Vol. 29, pp. 236-253).

5. Madison, C., Marquardt, V., Miller, B., Mueller, A., Pearce, S., Philion, C., ... \& Stainbrook, A. (2016). Sensory/Manipulation Interventions For Children with Autism and Developmental Disabilities: An Evidence-Based Practice Project

6. Thelen, A., Tiedt, E. M., Vang, S. C., Wagle, P. L., Weber, L. K., Wood, J. T., ... \& Zimmerli, B. M. (2016). Movement Interventions for Children with Autism and Developmental Disabilities An Evidence-Based Practice Project.

7. Masgutova SK, Akhmatova NK ,Sadowska L, Shackleford P and Akhmatov EA(2016) Neurosensorimotor Reflex Integration for Autism: a New Therapy Modality Paradigm, Journal of Pediatric Neurological Disorders, Volume 2 • Issue 1

8. Masgutova, S., \& Masgutov, D. (2015). Reflex Integration Disorder as a Ne w Treatment Paradigm for Children with Autism. A Collective Work. Florida, USA: SMEI, 171-180.

1. Masgutova, S., \& Sadowska, L. (2006). The Use of the Edu-K for Children with Learning Difficulties: Early Developmental Dynamic. In materials of: Polish National Conference: "NeuroKinesiology as the Method of Facilitation of Development and Learning for Children and Youth with Dyslexia, ADHD, Autism and Asperger (Vol. 8, No. 2006, pp. 39-50). 
2. Masgutova, S. K. (2000). NeuroKinesiological basis of the reflex integration for successful learning of the child. In materials of Polish National Conference: "NeuroKinesiology as the Method of Facilitation of Development and Learning for Children and Youth with Dyslexia, ADHD, Autism and Asperger." 08.04. 2006.(P1.).

3. Busz, T. E., \& Oginska-Dutkiewicz, B. (2015). Developmental Gains for a Child With Dyslexia and Allergies

1. Pilecki, W., Masgutova, S., \& Kowalewska, J. e t al.(2012) The impact of rehabilitation carried out using the Masgutov a neurosensorimotor reflex integration method in children with cerebral palsy on the results of brain stem auditory potential examinations. Adv Clin Exp Med, 21, 363-371

2. Pilecki, W., Kipiński, L., Szawrowicz-Pełka, T., Kałka, D., \& Masgutova, S. (2013). Spectral brain mapping in children with cerebral palsy treated by the Masgutova Neurosensorimotor Reflex Integration method. Journal of the Neurological Sciences, 333, e550

3. Pilecki, W., Pilecka-Kalamarz, A., Kalka, D., \& Kipinski, L. (2015). EEG Mapping Shows Changes in Brainwave Spectrum in Children with Cerebral Palsy During and After Masgutova Neurosensorimotor Reflex Integration Therapy

4. Masgutova, S., Russia-poland, P. D., Wenberg, E. S., \& Retschler, M. (2008). Masgutova Method of Reflex Integration for Children With Cerebral Palsy, 123.

5. Masgutova, S. (2008). Masgutova method of reflex integration for children with cerebral palsy. Svetlana Masgutova Educational Institute ${ }^{\circledR}$ for NeuroSensory-Motor and Reflex Integration, SMEI (USA) Posjećeno, 15, 2016

أمراض المناعة

1. Akhmatova, N. K. (2015). Immunological Effects of Masgutova Neurosensorimotor Reflex Integration in Children with Recurrent Obstructive Bronchitis. Int J Neurorehabilitation, 2(166), 2376-0281.

2. Akhmatova, N. K., Masgutova, S., Lebedinskaya, O. V., Akhmatov, E. A., \& Shubina, I. (2015). Immunological Efficiency of MNRI Program at Treatment of Respiratory Diseases. In Front. Immunol. Conference Abstract: IMMUNOCOLOMBIA2015-11th Congress of the Latin American Association of Immunology-10o. Congreso de la Asociación Colombiana de Alergia, Asma e Inmunología. doi: 10.3389/conf. fimmu (Vol. 72

متلازمة داون

1. Masgutova, S., Akhmatova, N., \& Ludwika, S. (2016). Reflex Profile of 
Children with Down Syndrome Improvement of Neurosensorimotor Development Using the MNRI® Reflex Integration Program. Int $J$ Neurorehabilitation, 3(197), 2376-0281

2. Akhmatova, N., \& Akhmatova, E. (2017). Influence of MNRI on the Immune Status of Children with Down Syndrome. J Clin Cell Immunol, 7(483), 2.

3. Svetlana Masgutova, Ludwika Sadowska, Joanna Kowalewska, Denis Masgutov, Nelli Akhmatova and Henryk Filipowski (2015). Use of a Neurosensorimotor Reflex Integration Program to Improve Reflex Patterns of Children with Down Syndrome, Journal-Of-Neurology- No. $4: 59$

Akhmatova, N. K. (2015). Immunological Effects of Masgutova Neurosensorimotor Reflex Integration in Children with Recurrent Obstructive Bronchitis. Int J Neurorehabilitation, 2(166), 2376-0281.

اضطرابات اللغة والكلام

1. Masgutova, S. K., \& Regner, A. (2008). Language development using sensory-motor integration approach. Scientific edition: Prof. T. Galkowski, Dr. B. Dolyk. Warsaw: Continulo

2. Masgutova, S. (2005). Reflexes as the Basis of the Nerve System Development and Formation of the Motor Patterns in Infancy. Materials of International Conference: Modern Methods of Stimulation of Motor and Language Development. International Kinesio-Rehabilitation Camp for Children with Challenges of Dr. S. Masgutova Institute, MINK. Warsaw

المشكلات السمعية البصرية الماية

1. Masgutova, S. (2009). MNRI® Neurosensorimotor Development: Visual and Auditory Reflexes Integration. Facilitation Program of Development and Learning for Children and Adults. Florida, 86.

2. Masgutova, S. K. (2007). Neuro-Sensory-Motor Development: Visual and Auditory Reflexes Integration. Facilitation Program of Development and Learning for Children and Adults. Warsaw, Poland.

اضطرابات الصدمة وما بعد الصدمة

1. Masgutova, S., \& Masgutov, D. (2015). Reflex Integration for Trauma Recovery MNRI ${ }^{\circledR}$ Stress Resilience Introductory Program. Orlando: SMEI.

2. Masgutova, S. (2018). Post-Trauma Recovery in Children of Newtown, CT using MNRI Reflex Integration. Journal of Traumatic Stress Disorders \& Treatment, 2017.

الموهويون

Masgutova, S., \& Masgutov, P. D. D. (2015). A Search for Excellence in Gifted Children with Reflex Integration. 


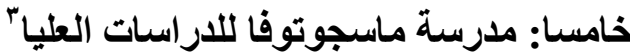

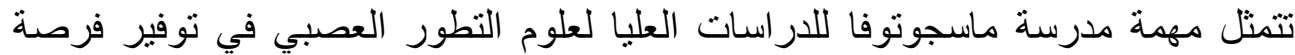

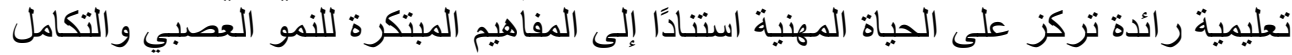
العصبي الحسي الحركي للمنعكسات.

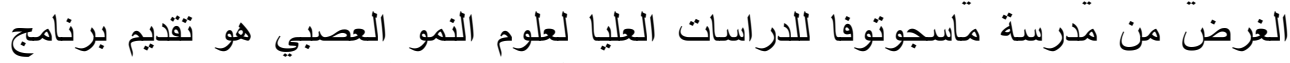

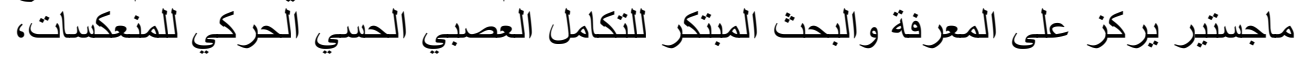
و التعديل العصبي ، والنمو العصبي لتحسين الرفاهية الجسدية والاجتماعية والمعرفية لليكية

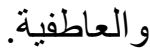

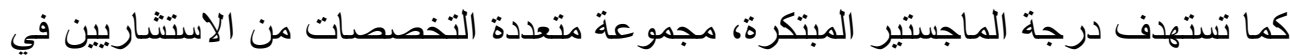

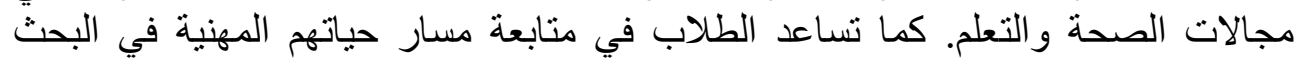

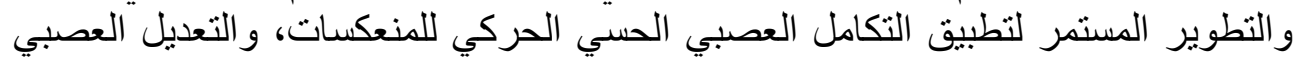

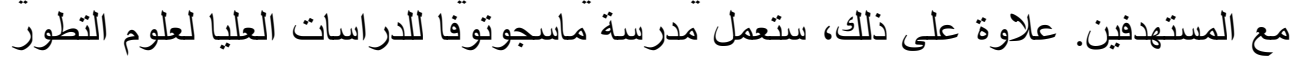

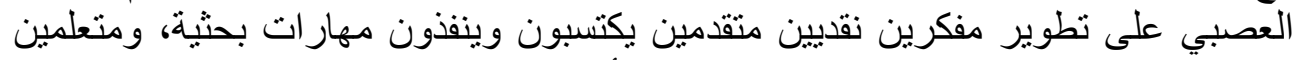

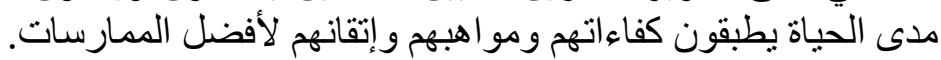

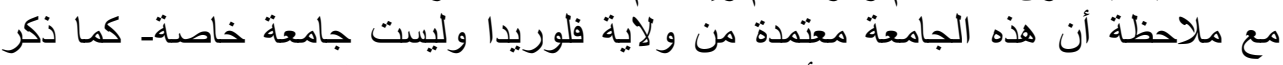

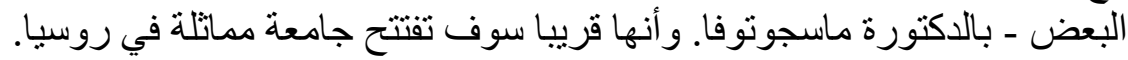

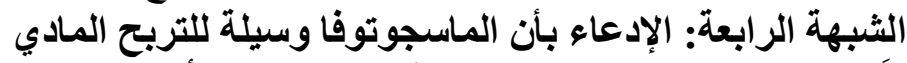

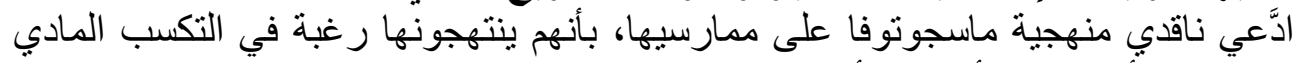

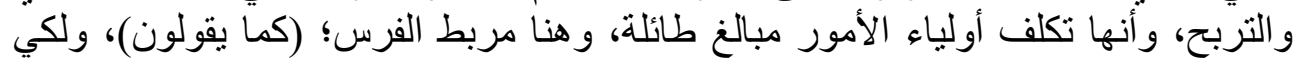

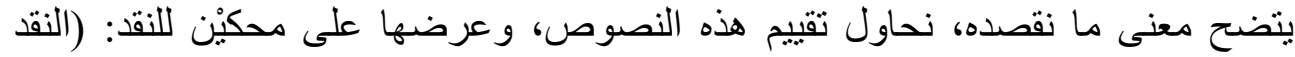

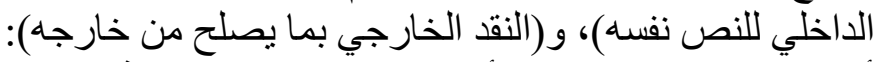
أولا: النقل الخارجي: حول أسعار جلسات الماسجوتوفا

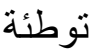

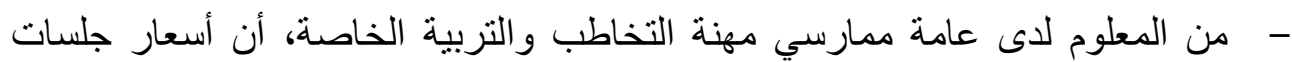

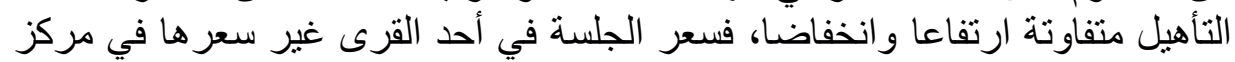

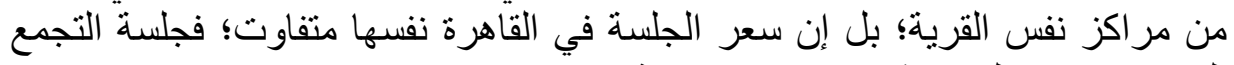

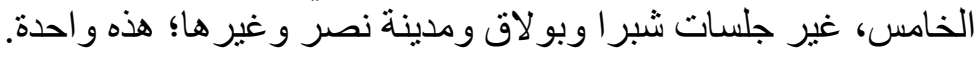

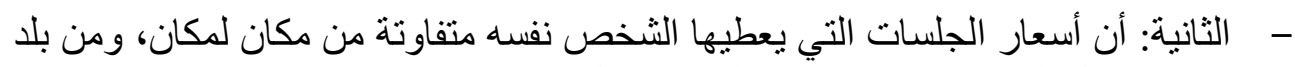

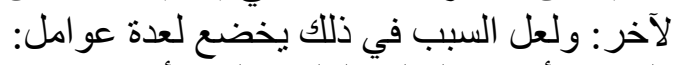
أو لا: لتفاوت أسعار التكلفة الملقاه على الأخصائي، من إيجار ورواتب وخلئ وخلافه.

/https://www.linkedin.cn/company/mgsns - 
ثانيا: تكاليف الإنشاء و التأثثيث؛ فالثخص الذي كلَّف مائة ألف جنيه في إنشاء مكانه، غير من كلفه مئات الآلاف. ثالثا: مستوى دخول الأفر اد؛ فالفرد الذي جملة دخله في الريف مثنلا ألف جنيه شهريا؛ غير

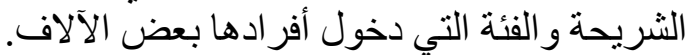

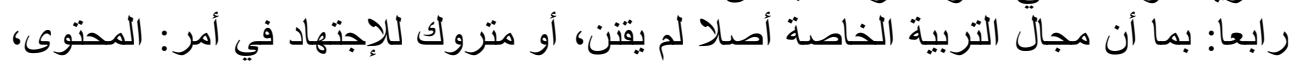
و المعايير ؛ سيظل أيضا بلا تقنين في مجال الماليات.

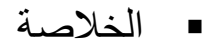

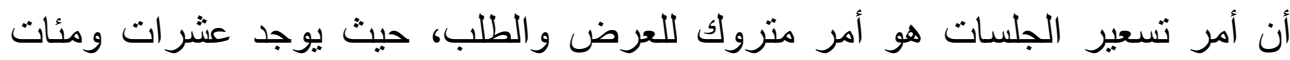

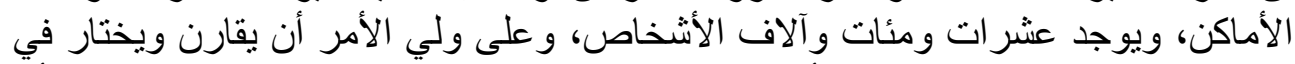

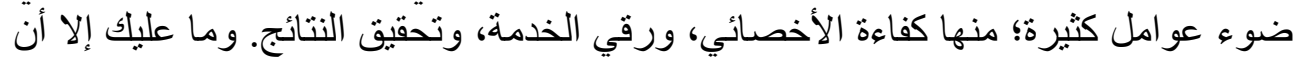
تختار ما تريد.

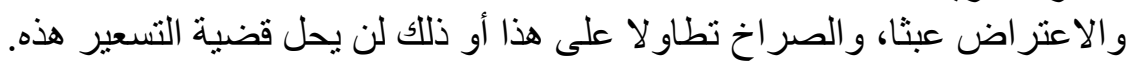

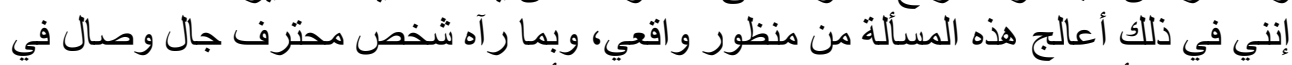

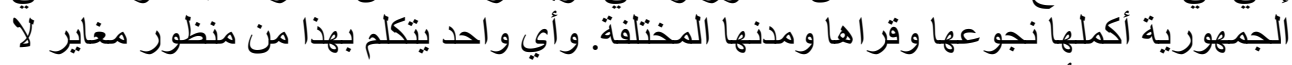

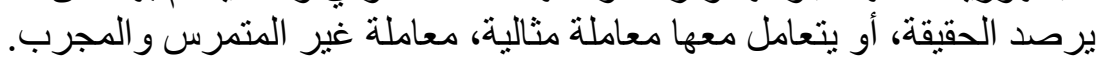

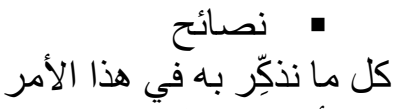
ل أن مهنة التربية الخاصة مهنة سامية، و أنها مهنة إنسانية في المقام الأول، وأنها ليست سبيلا للتربح و التكسب على لتى حساب معاناة الأفر اد.

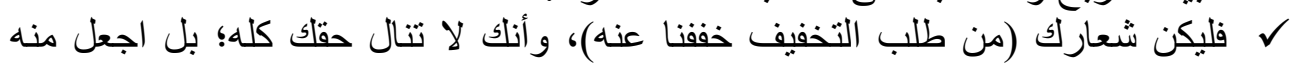

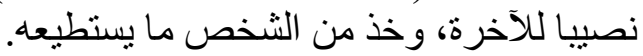

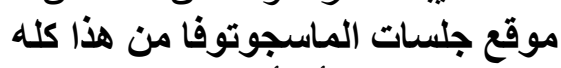

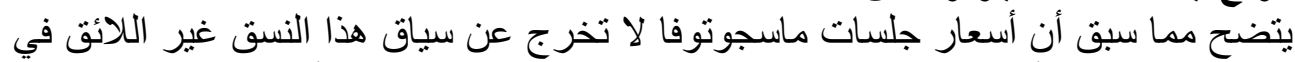

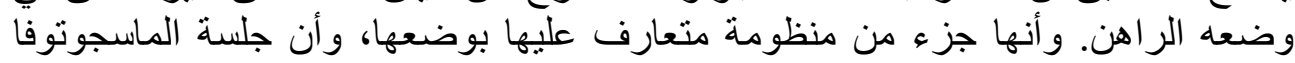

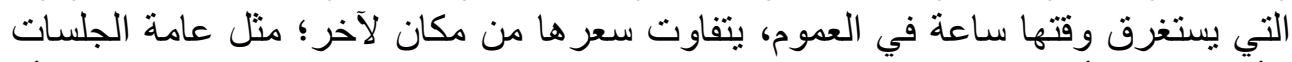

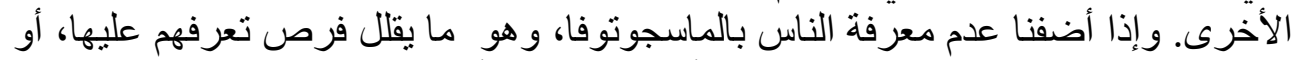

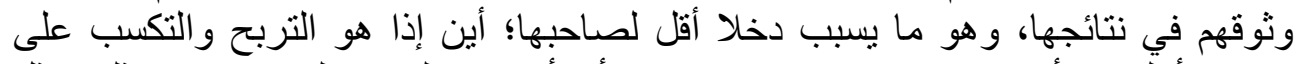

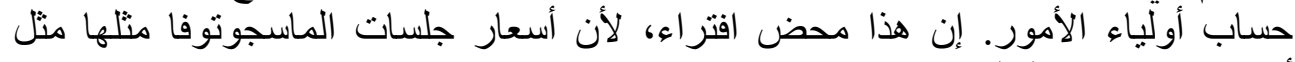
أسعار غير ها من الجلساء الأورن.

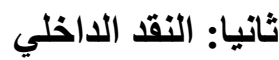
يلاحظ من كلام الثخص الثيا: الناقد، وتكرار كلامه على نفس النقطة بألفاظ متباينة حول أسعار

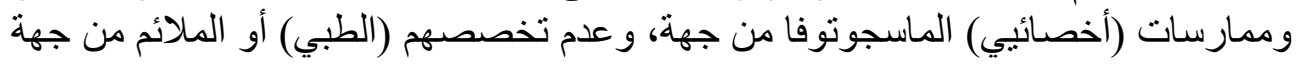


أخرى، أن التخوُّف الأساسي ربما يكمن في بروز فئة جديدة من فئات الأخصائيين الذين

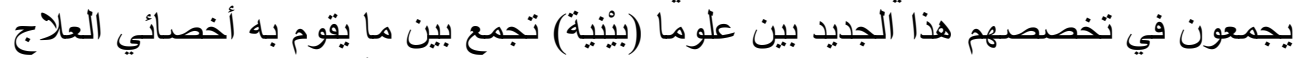

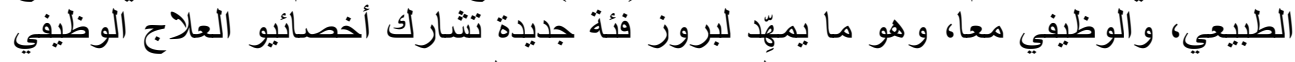

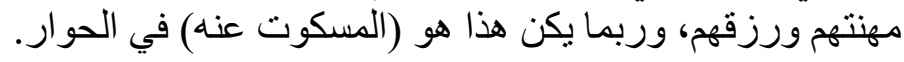
ما معنى هذا؟

نحن نعلم جيدا أن هنالك خلافا كبيرا بين ممارسي العلاج الوظيفي، وممارسي العلاج

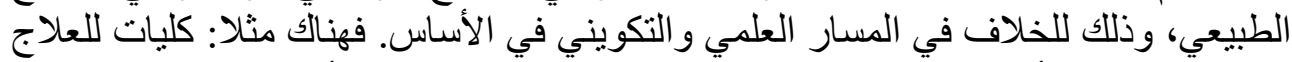

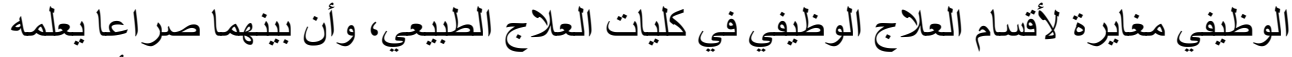

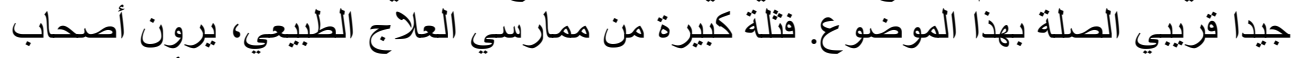

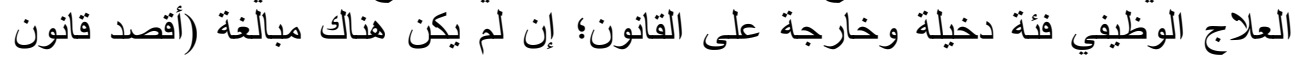
العرف الطبي الثنائع).

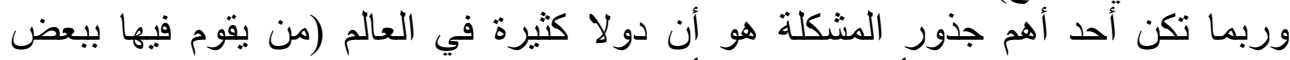

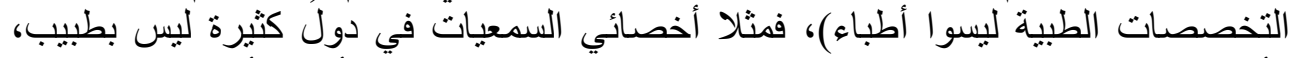

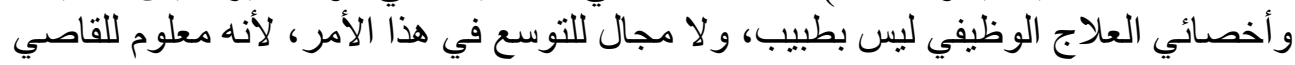
و والداني.

أضف إلى ذلك المشكلة الكبرى، و الكارثة الأمرّ، و هو أن عشر ات الدورات التي تعطي يوميا

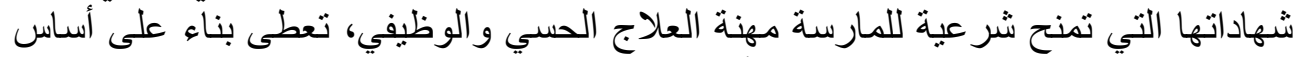

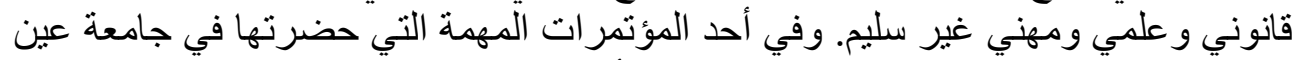

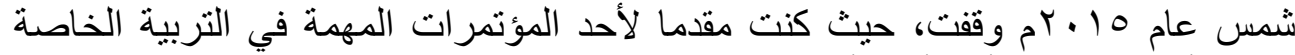

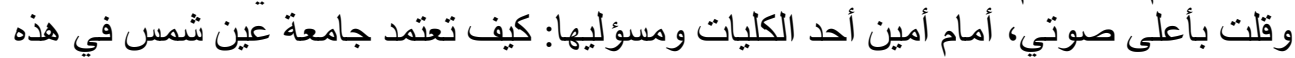

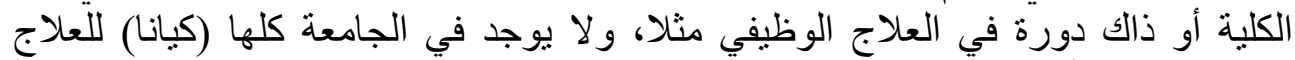

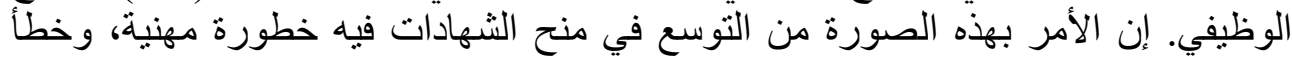

الثبهة الخامسة: الإدعاء باستبعاد كافة أنماط التدخل الأخرى مع جلسات ماسجوتوفا

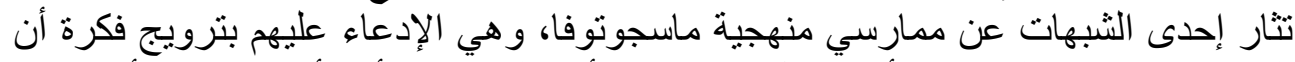

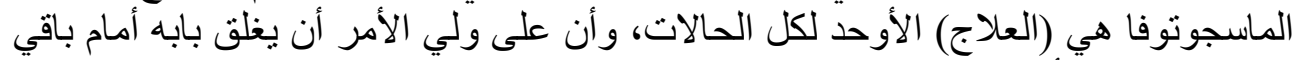

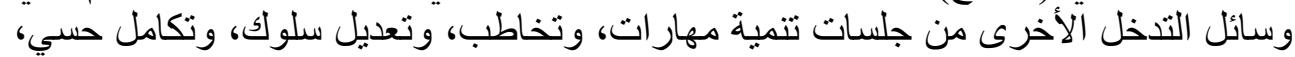

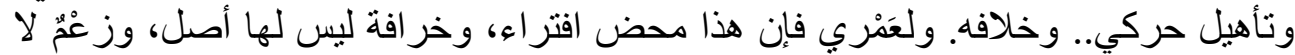
يُبنى على دليل، ويمكن الرد عليه من ثملاث زئن زو ايا: 
الأولى: على المستوى المركزي

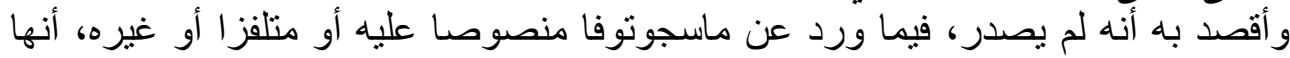

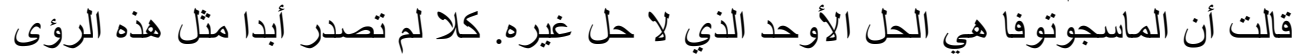

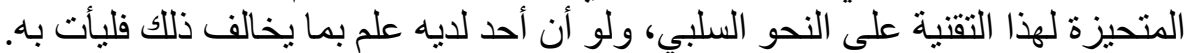

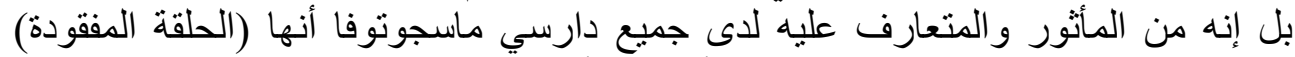

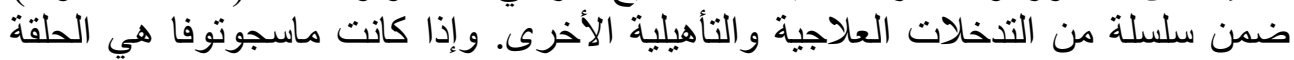

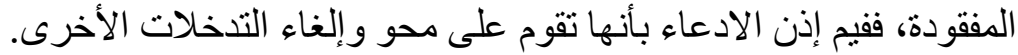

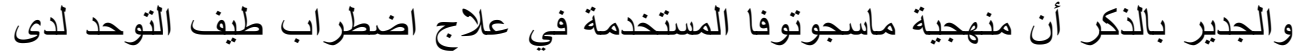

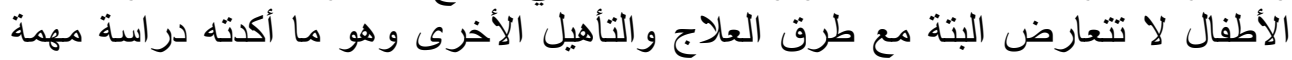

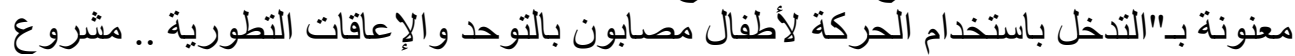
Thelen, A., Tiedt, E. M., Vang, S. C., Wagle, P. ) تدريبي مبني على الألى الأدلة (L., Weber, L. K., Wood, J. T., ... \& Zimmerli, B. M. 2016)

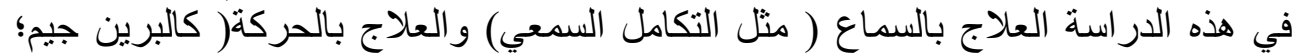

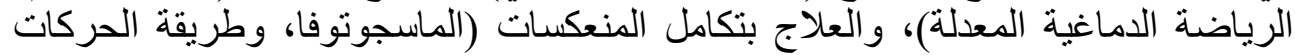

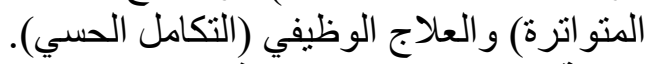

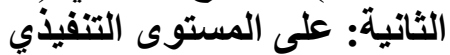

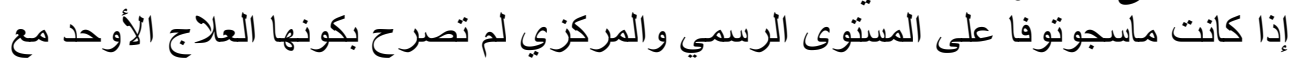

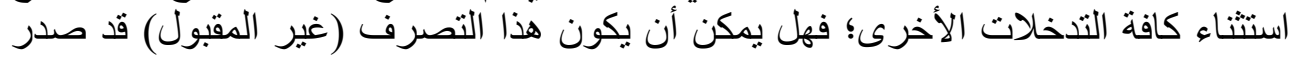

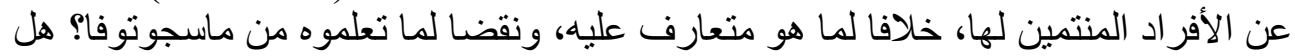

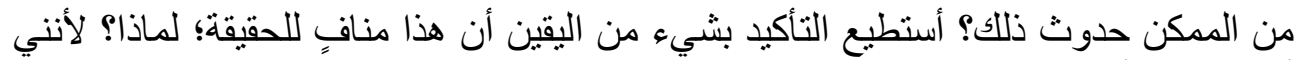

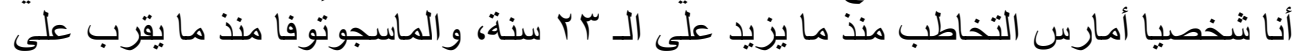

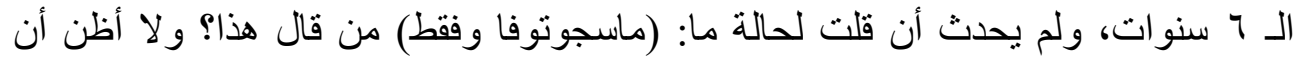

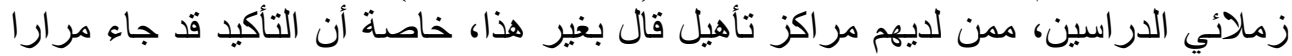

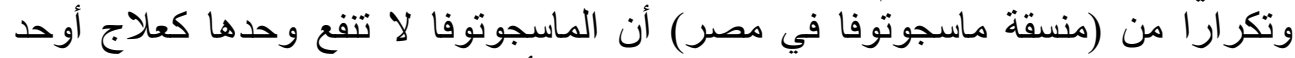

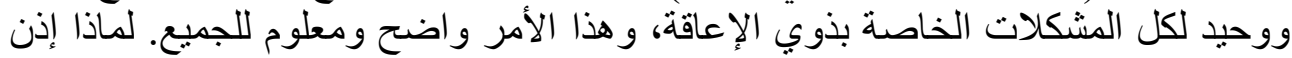

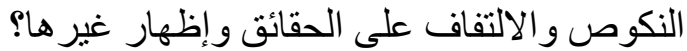

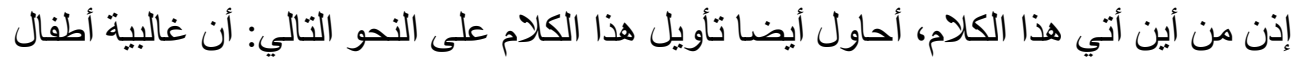

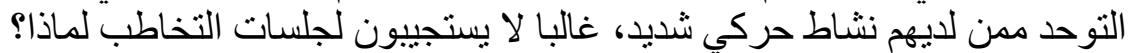

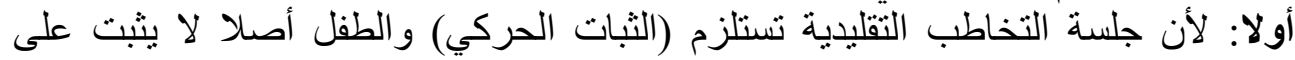
ثانيا: لأن جلئ الأنة التخاطب، أو التعامل مع اللغة يستلزم من الطفل أن (يتو اصل بصريا) مع

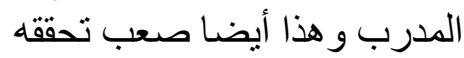


ثالثا: أن الطفل ليس لديه انتباه أو تركيز يسمح له بالاستمرار فترة طويلة كي يتلقى جلسة التخاطب.

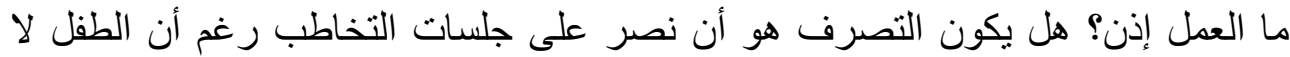

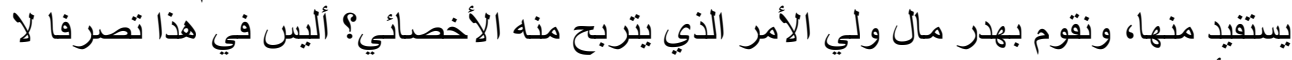
يليق أبدا؟

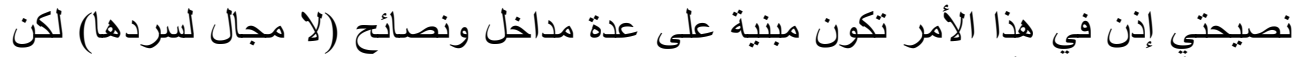

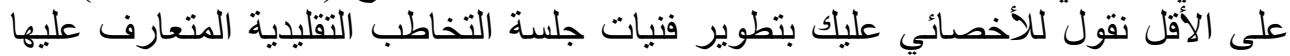

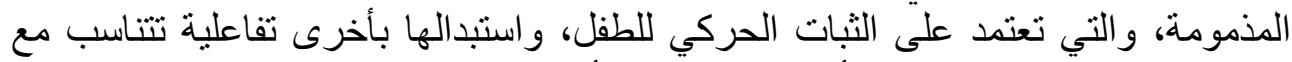

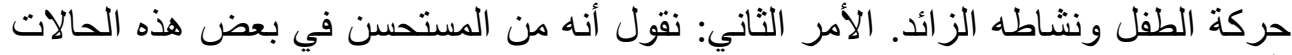

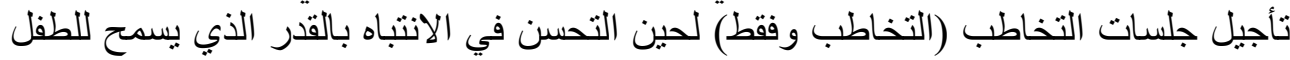

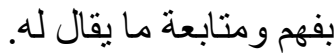

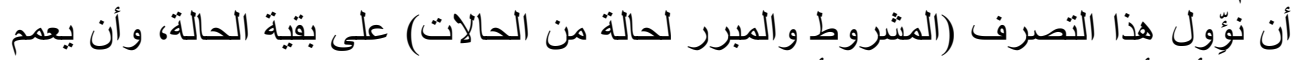

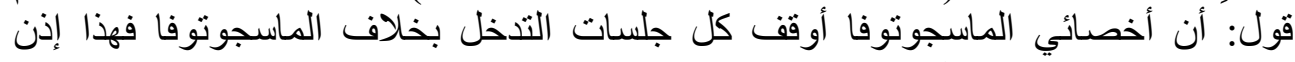

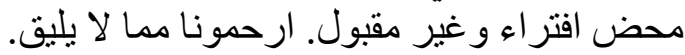

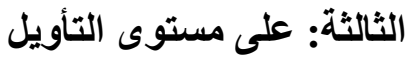
على اعتبار جواز صحة صنالثة هذا القول: أنه صدر من أحد، أو عدد من من ممارسي منهجية

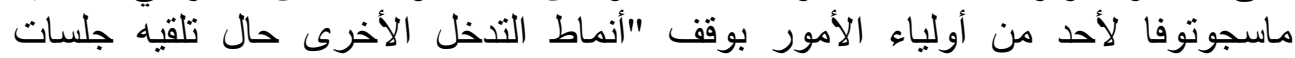

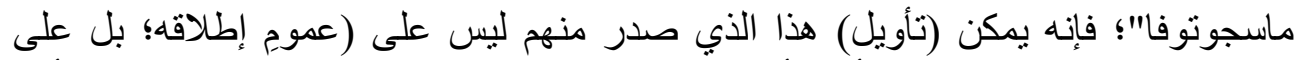

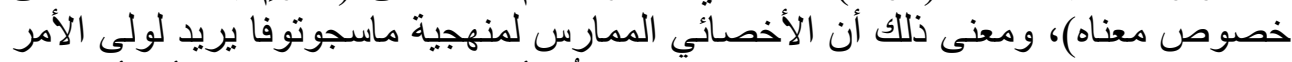

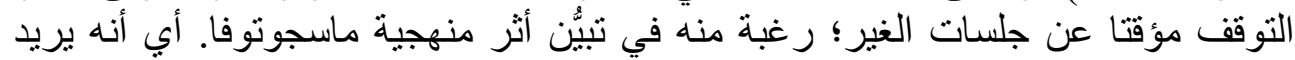

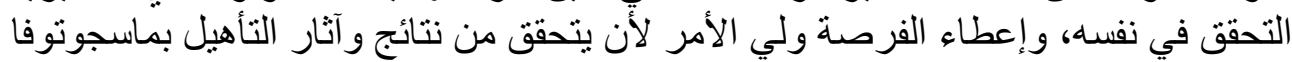

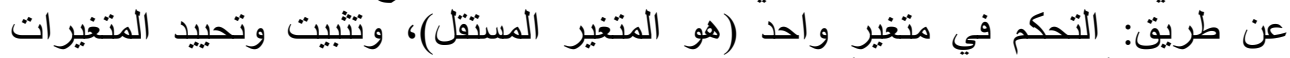

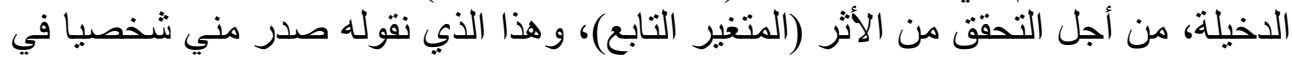

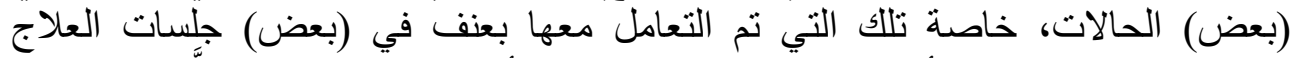

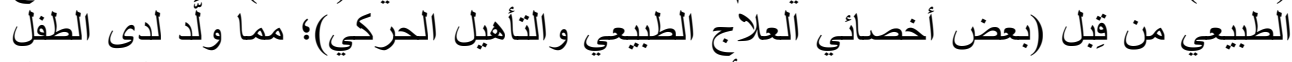

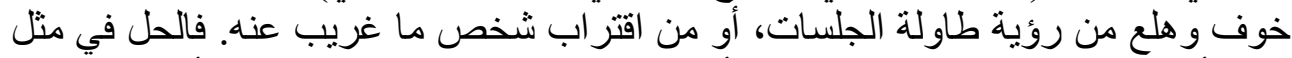

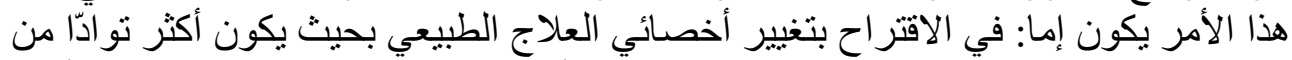

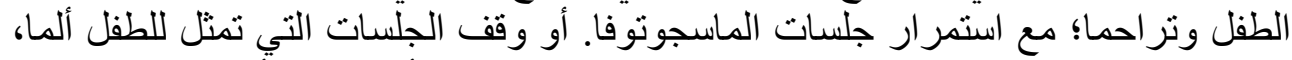

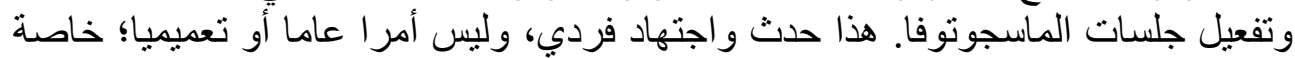

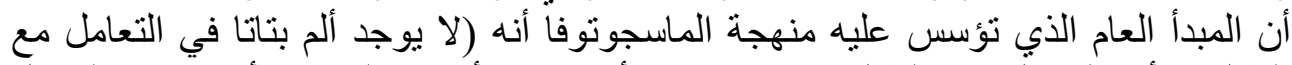

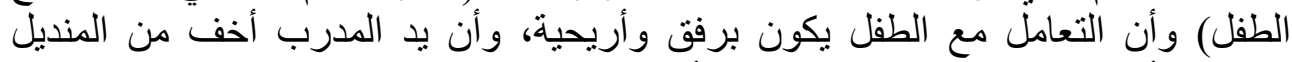
الورق، وأن التسبب في إحساس الطفل بالألم يجعل من الجهاز العصبي مقاوم بطبعه للشغل 
و التفاعل مع التدريبات؛ وهو ما يقلل استجابته، لأن نظرية الماسجوتوفا تتعامل مع (الجهاز

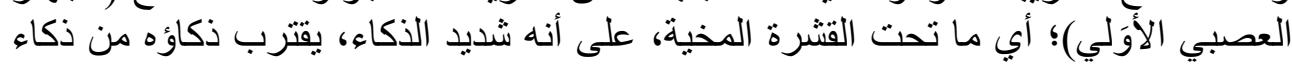

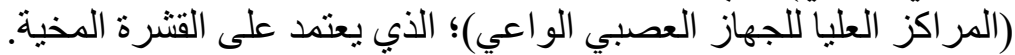

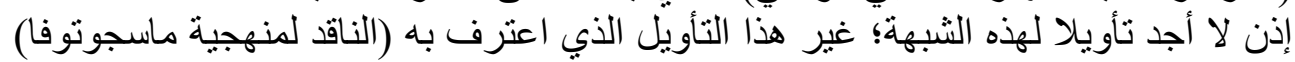

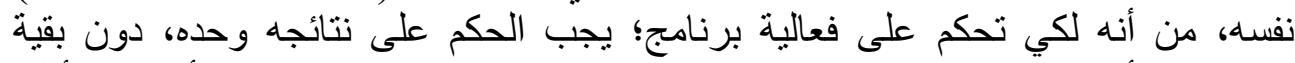

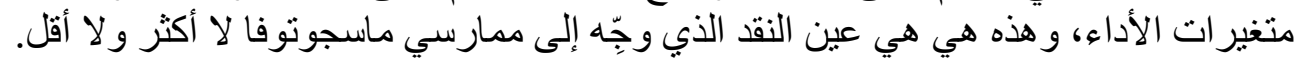

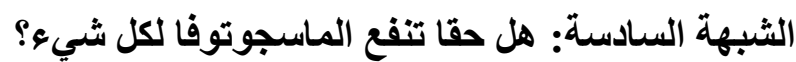

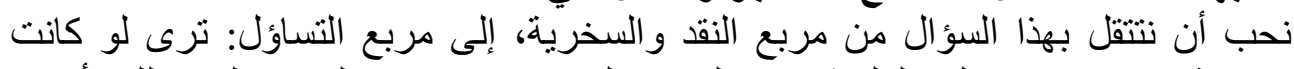

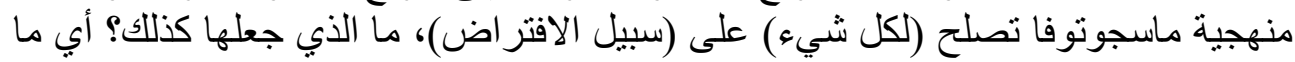

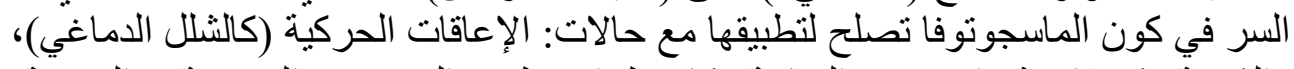

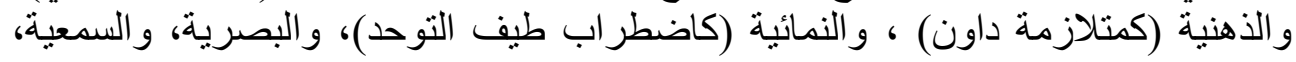

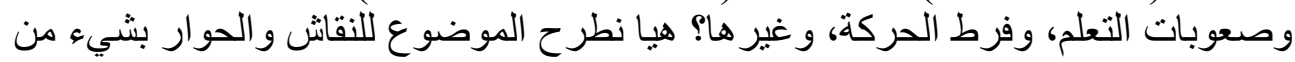
الو أوعي: - الإطعات

أولا: الإطلاق المشروط

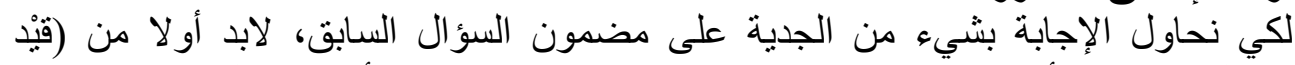

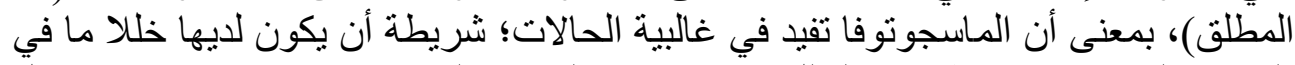

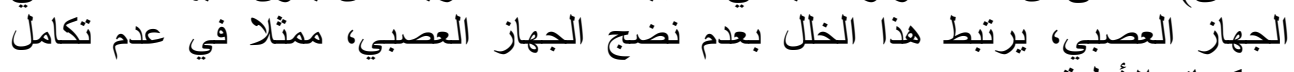
منعكساته الأولية. أظن أننا بهذا (الإطلاق المشروط) ربما نخفف من حدة النقد الموجه إلى منهجية ماسجوتوفا، لماذا؟

هذا ما سنعر فه في النقطة التالية: ثانيا: خلل الأساس والبنية: الفئة يؤدي إلى خلل البناء بأكمله

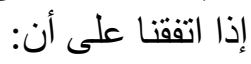
(1) جميع القدرات المعرفية، والحركية، والتهات واللغوية، و المهارية، و الوجدانية، والأكاديمية .. مرتبطة بسلامة الجسم وصحته الفئه

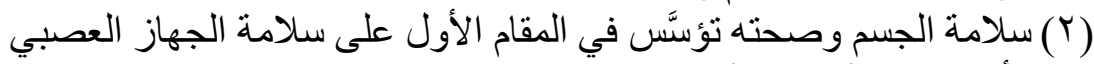

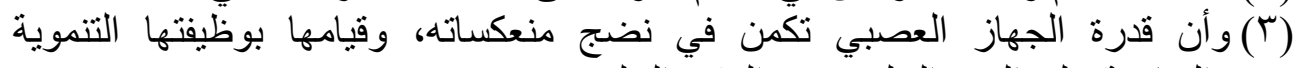

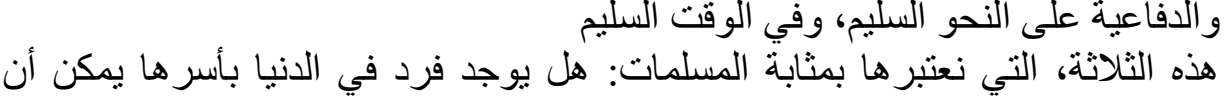

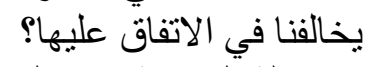
في حالة المو افقة، فإننا لن نزيد إلا أن نضيف على هذه الثناثة، رابعة هي: 


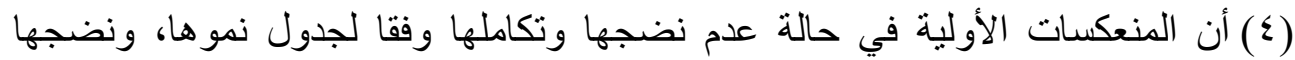

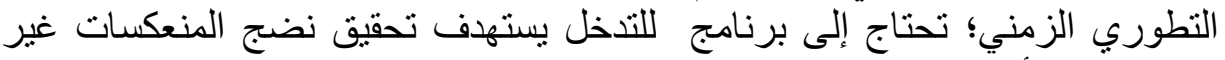

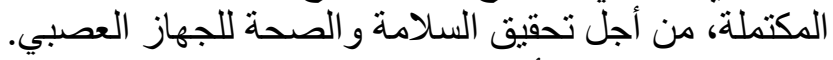

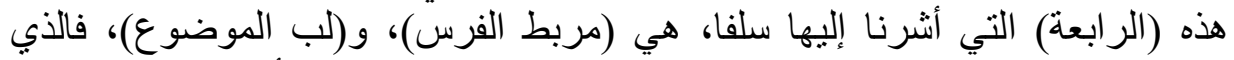

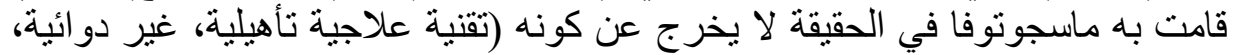

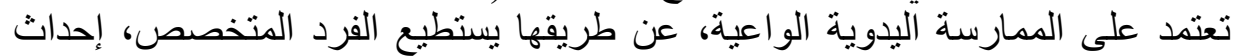

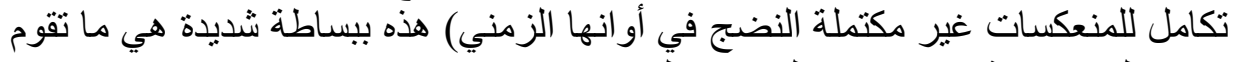

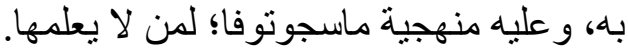

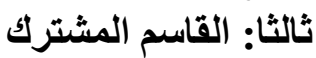

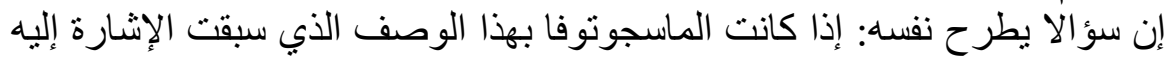

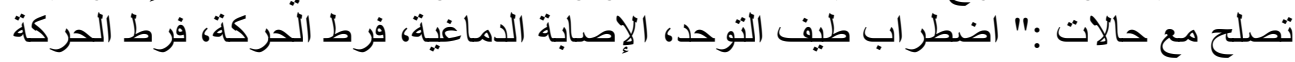

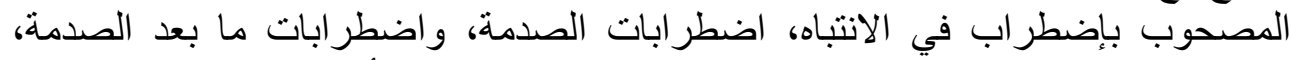

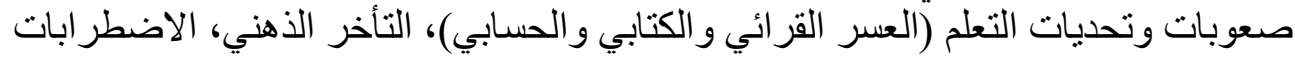

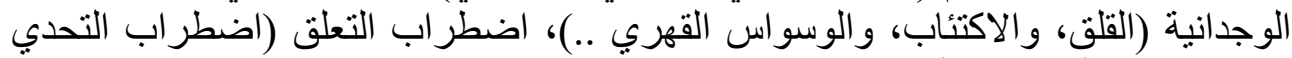

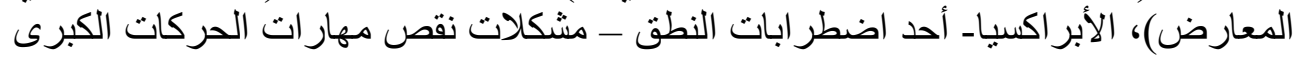

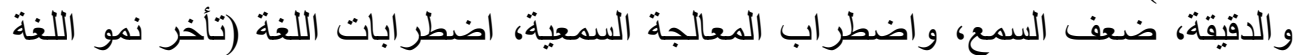

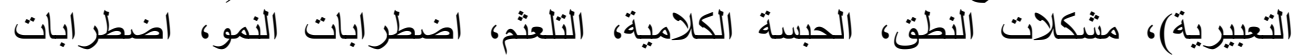

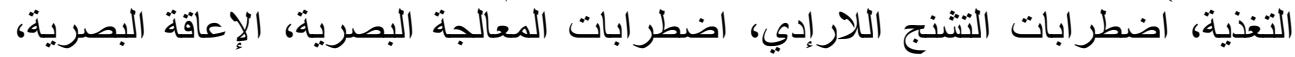

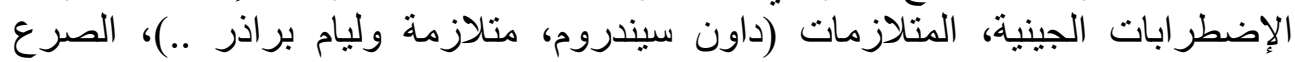

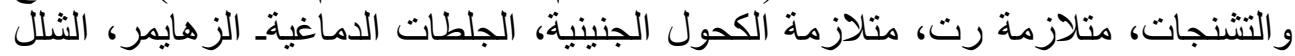

الرعاش(MasgutovaS.(2015).

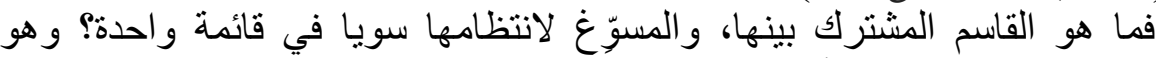

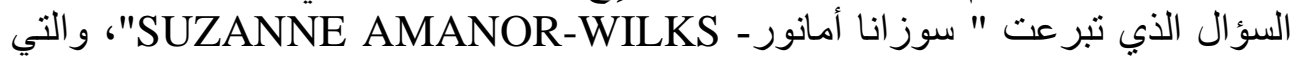

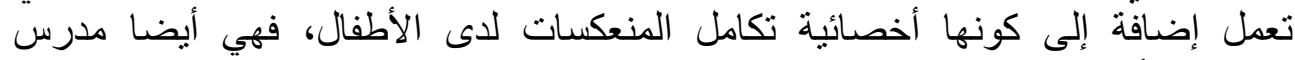

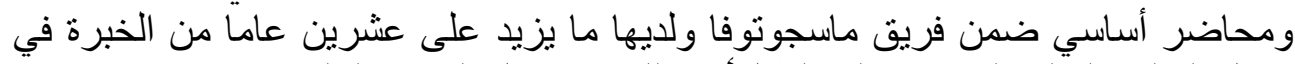

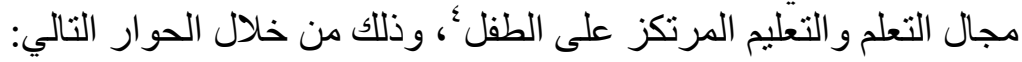

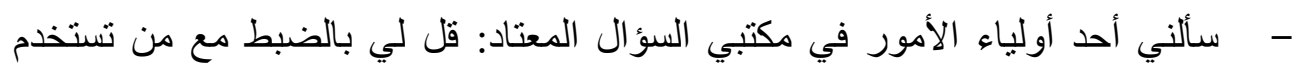

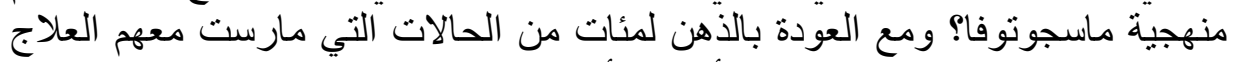

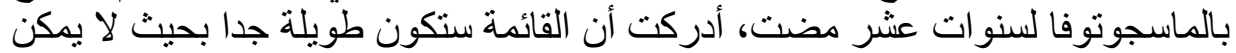
تعدادها.

${ }^{4}$ - http://www.itcc3.com/ 
- مبإيتسامة يصحبها بريقُ يملؤ العينين أجبت: منهجية ماسجونوفا لتكامل رد الفعل فئل

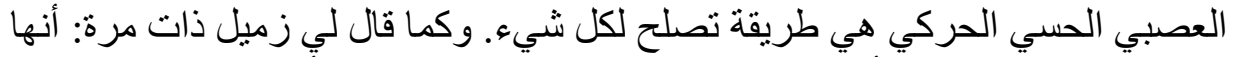

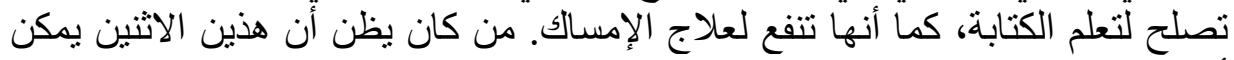

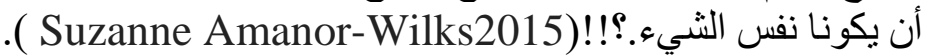

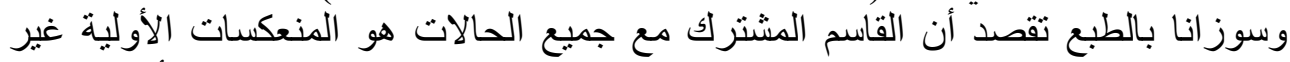

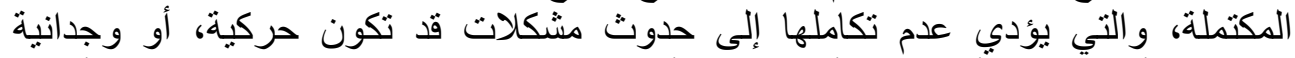

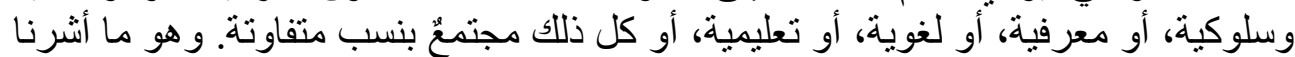

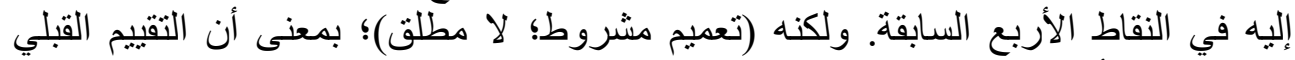

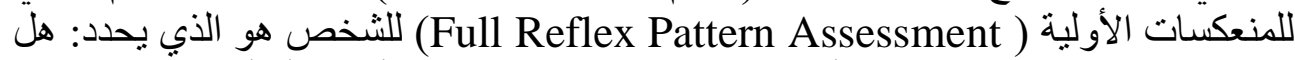

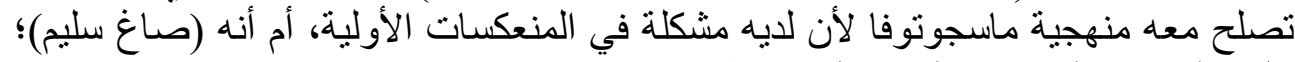

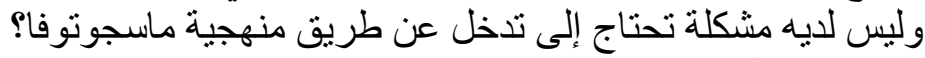
تفرد منهجية ماسجوتوفية

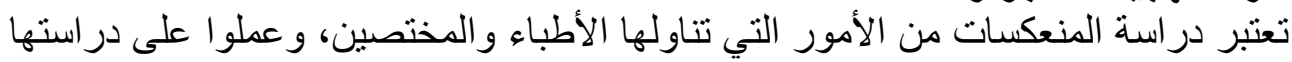

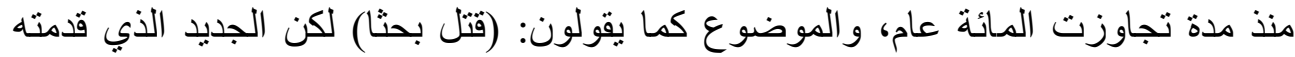

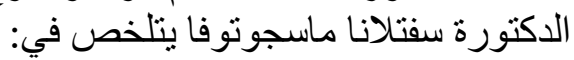

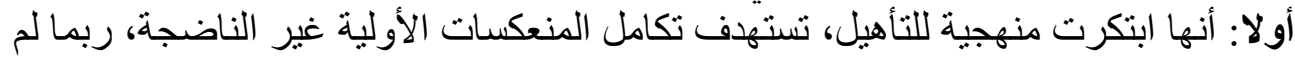
يسبقها إليها أحد، وذلك لأن أن كل العلماء الذين تحدثوا عن المنعكسات، وآلية اختبار ها؛ لم الن يوضحو ا كيف يكون تكاملها.

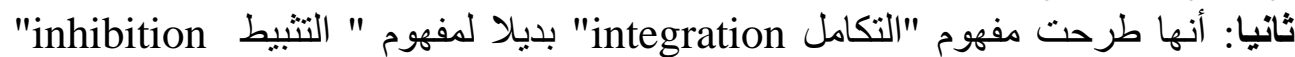

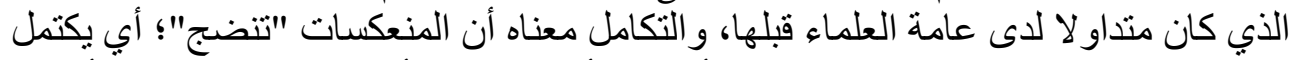

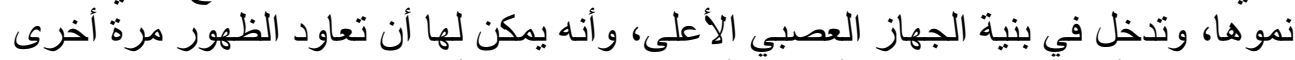

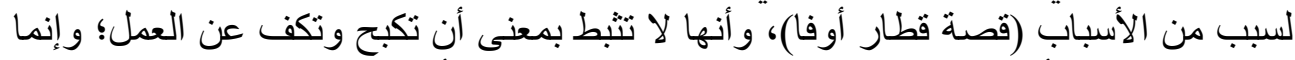

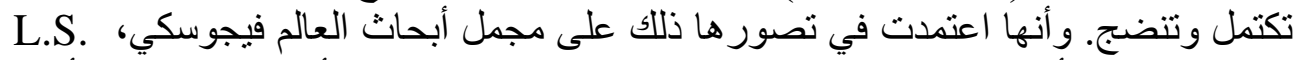
Vygotsky,

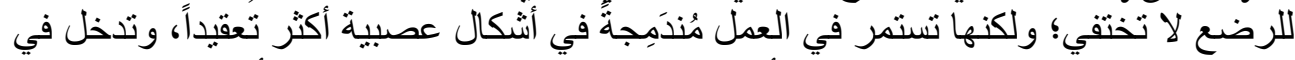

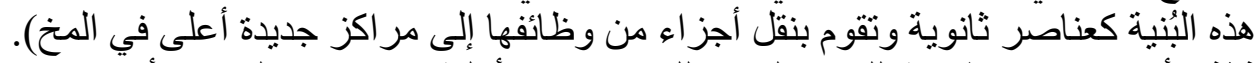

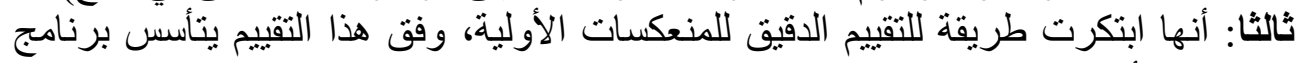

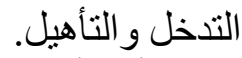

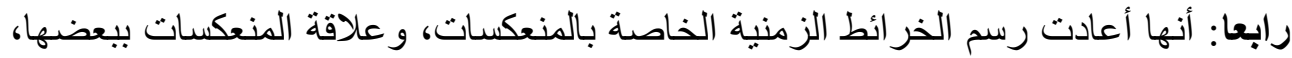

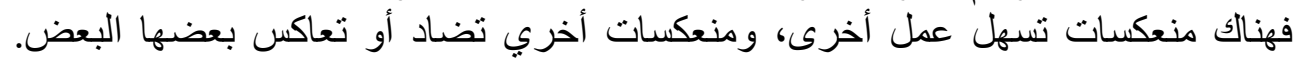

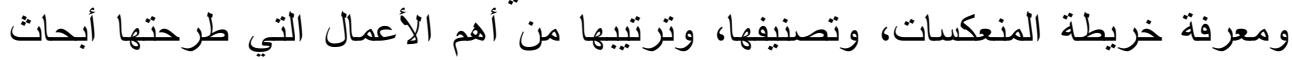
ماسجوتوفا. 
خامسا: أنها اكتشف، أو وصَّفت، أو عدَّلت في توصيف، أو قامت بتسمية ونوثيق مجمو عة منعكسات جديدة، لم يسبق أن تناولها العاء العماء قبلها، مثل:

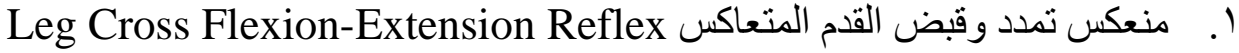

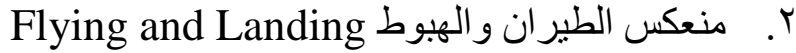
r.

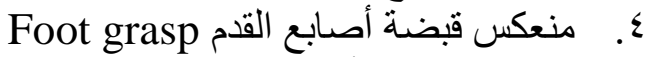

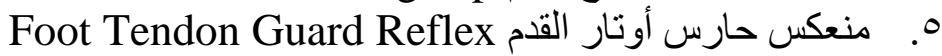

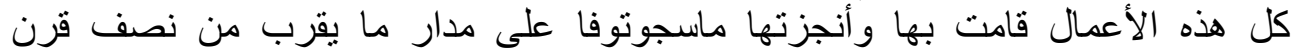

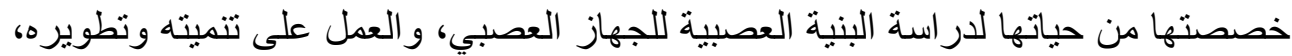

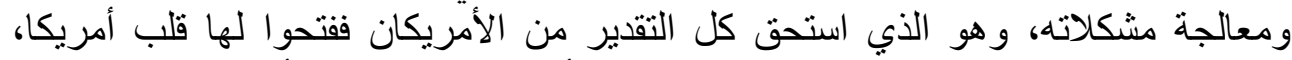

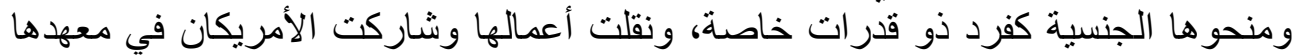

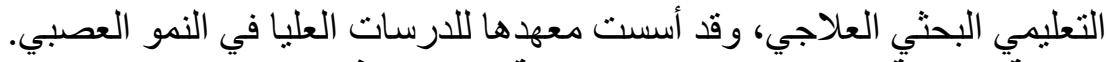

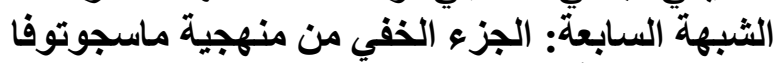

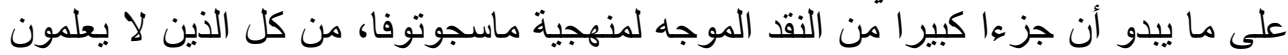

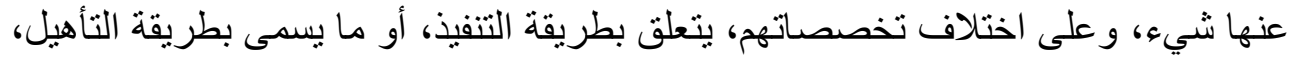

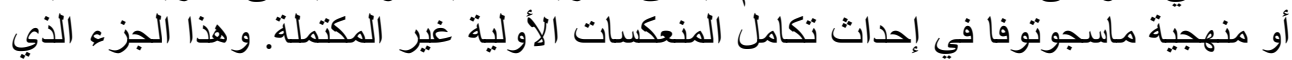

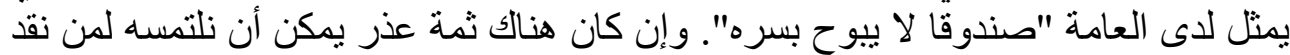

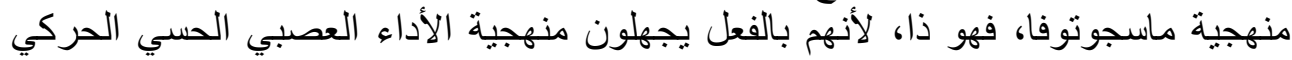

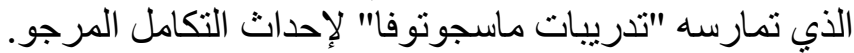

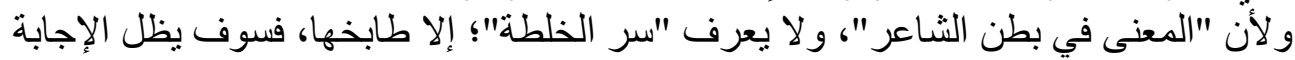

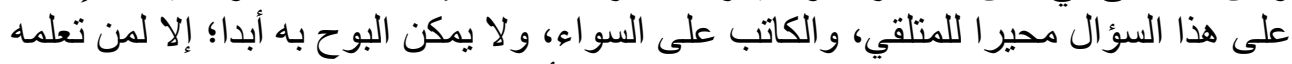

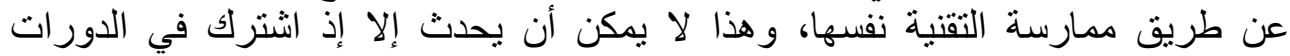

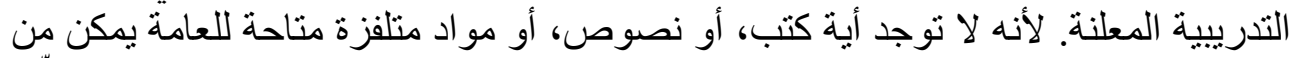

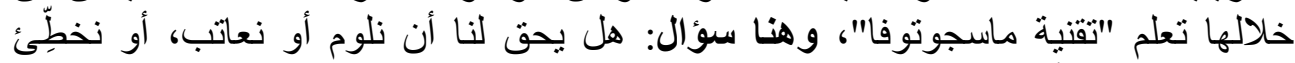

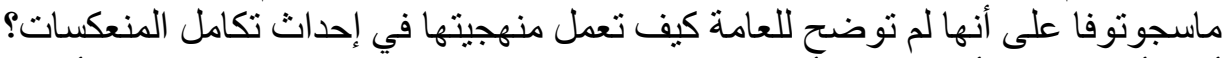

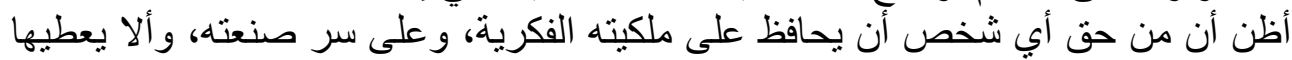

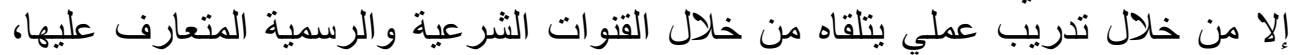
يتأكد فيها صناحب الحق، أن المشترك قد اكتسب المهارة المرجو له تلعلمها، وتطبيقها.

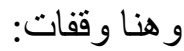
أولا: التأكيد على أن ما نشر، وما عرضات عرض من أبحاث، وعلى المواقع.. بشتى أنواعه، لم

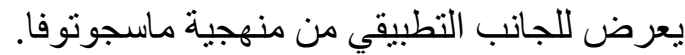

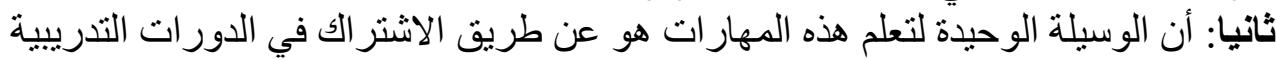




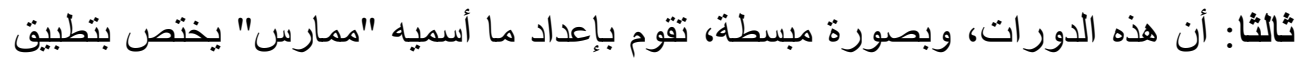

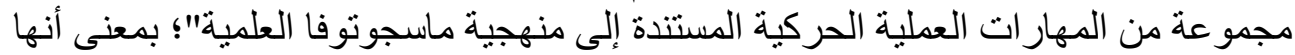

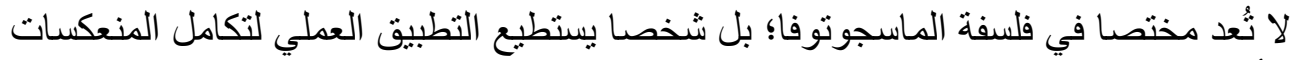

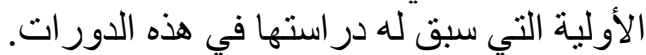

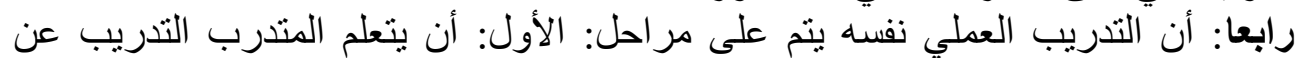

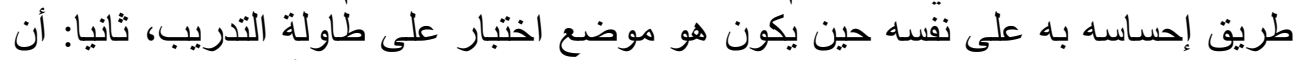

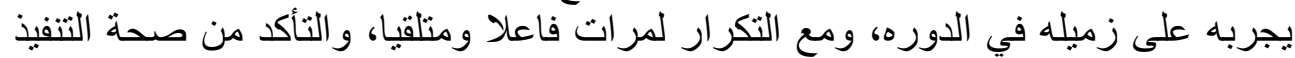

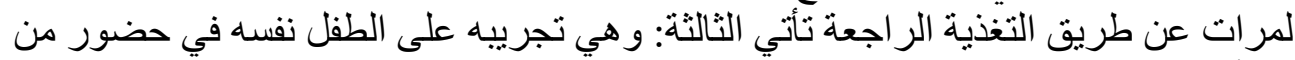
هو أكثر منه خبرة. خامسا: أن هناك متابعة ومر اجعات مستمرة للجانب العملي للتأكد من كون الثخص الذي الذي

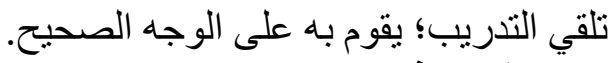

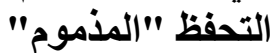

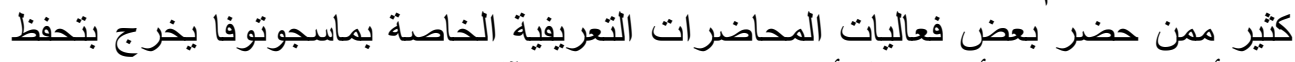

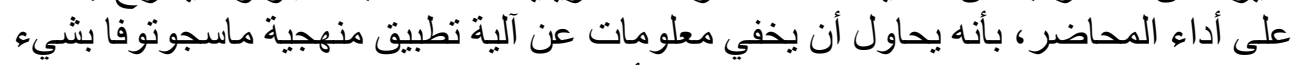

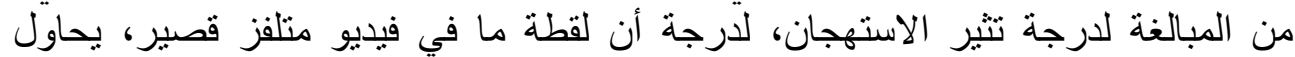

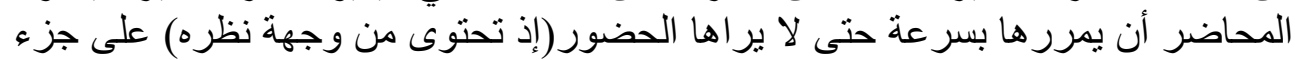

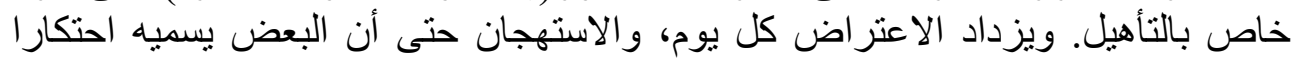

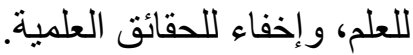

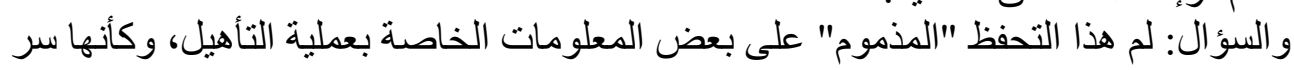

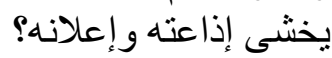

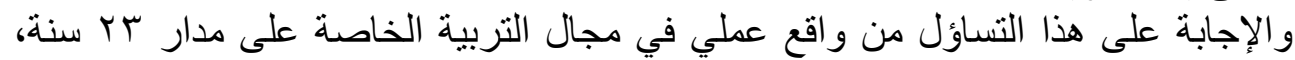

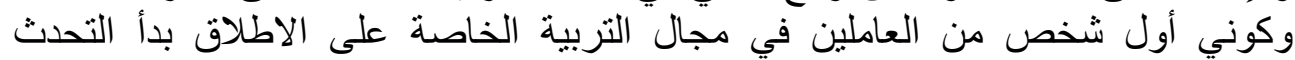

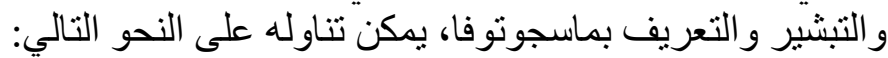

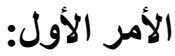
مجال لا رب له ولا سيّّ؛ أقصد مجال التربية الخاصة (وقد كتبت في هذا مرارا) هذا المجال

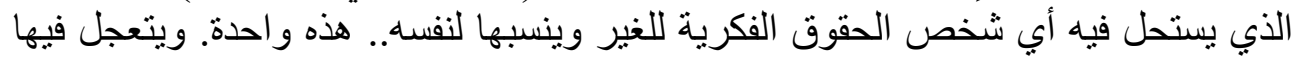

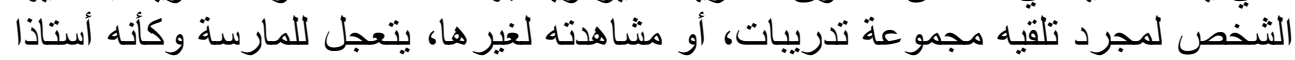

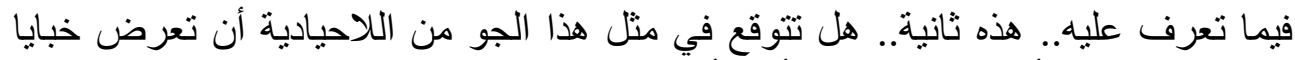

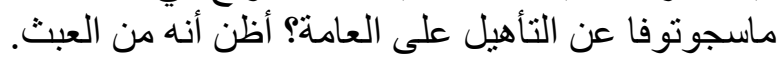
حكاية مانجوتية

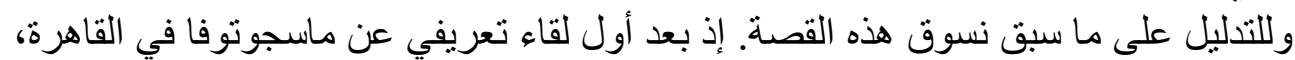

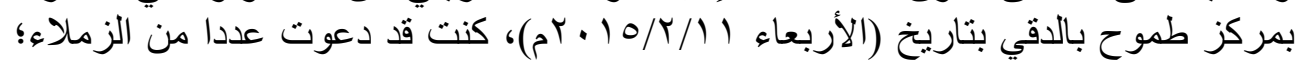




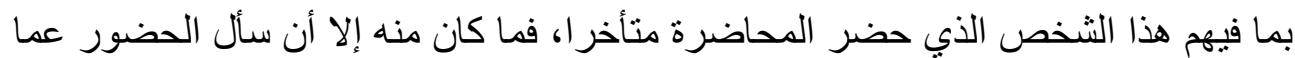

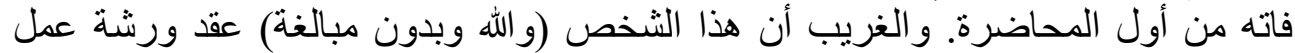

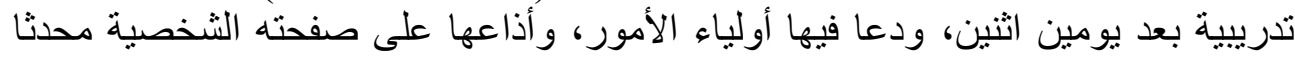

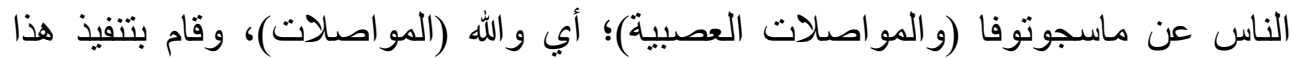

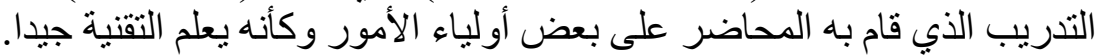

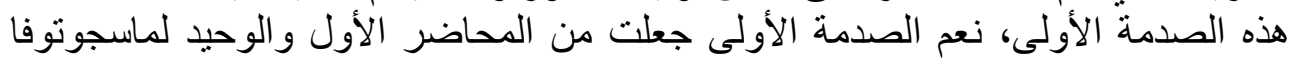

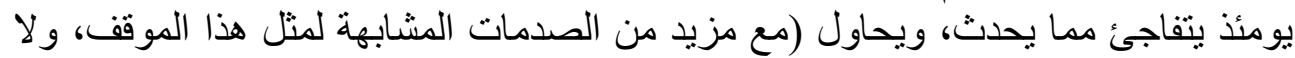

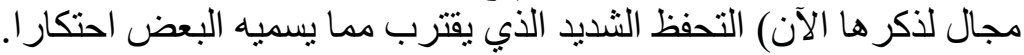

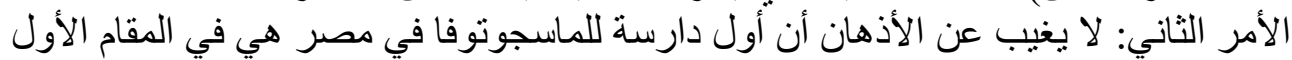

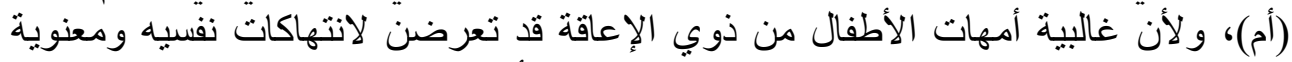

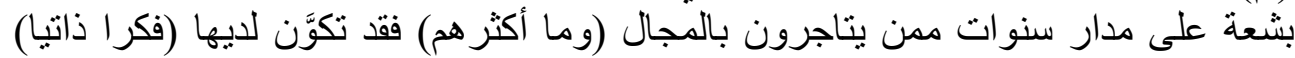

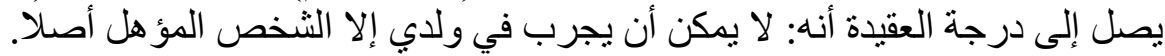

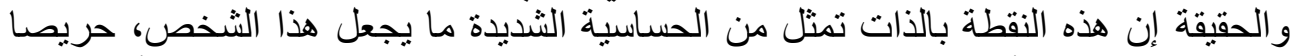

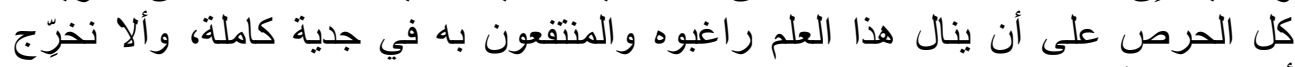
أنصاف متعلمين.

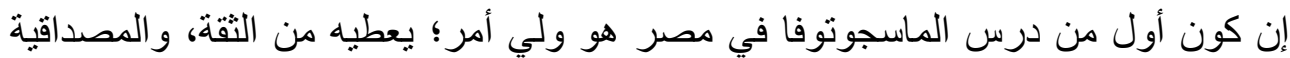

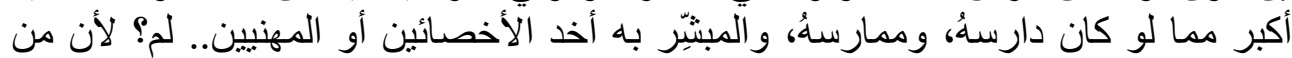

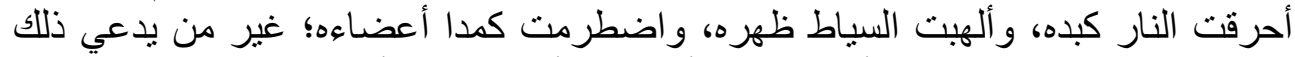

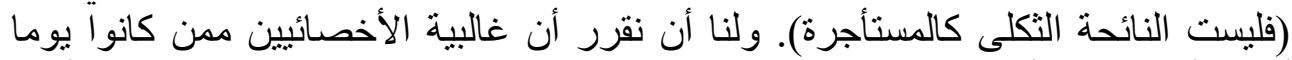

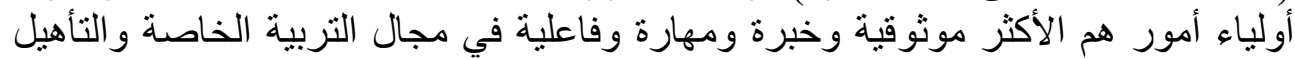

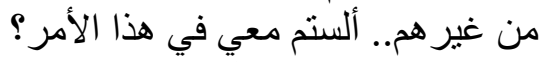

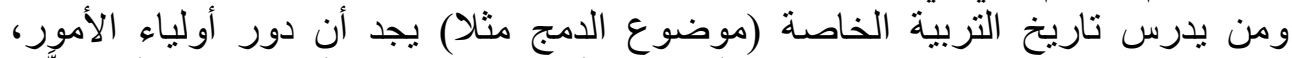

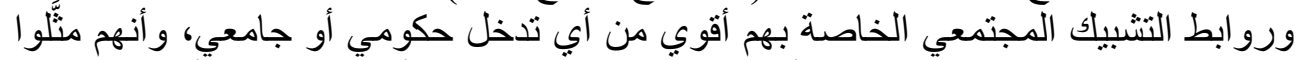

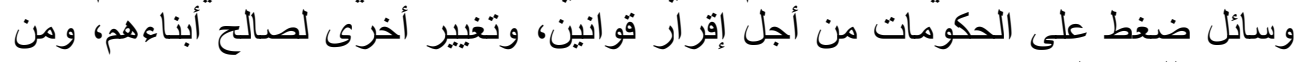
بر اجع ذللك يدرك صحتئه بالله عليكم ليقل لي واحد منكم: كيف لولي لئه أمر عانى الأمرين من وليده، وسافر به دول العال العالم

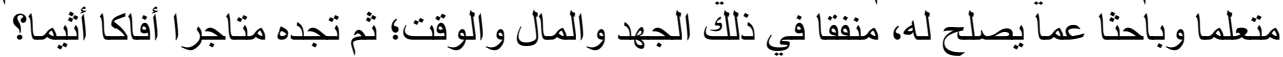

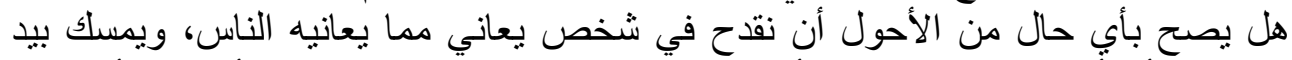

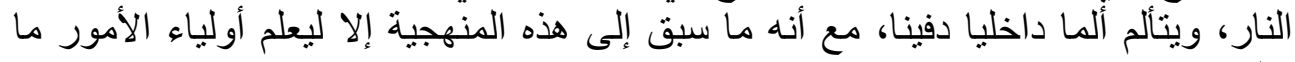
تعلمه.

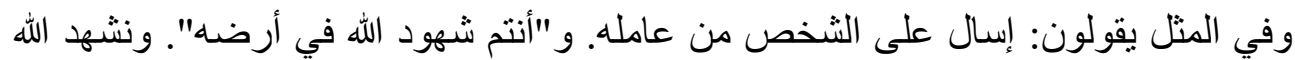

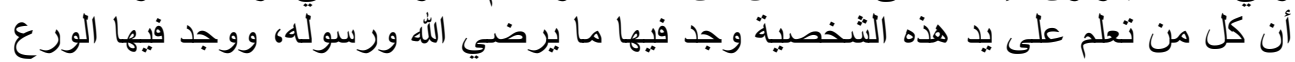




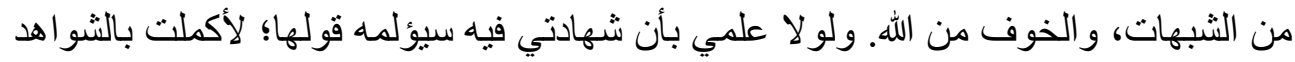
و المواقف ما يؤكد ذلك. ولكن في الإشارة ما يغني عن العبارة، وفي التلميح ما يغني عن فئه التصريح.

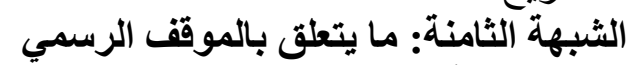

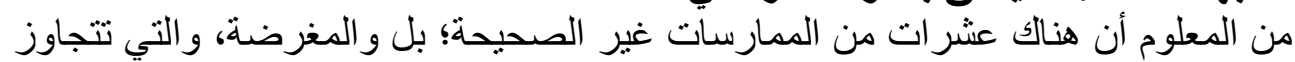

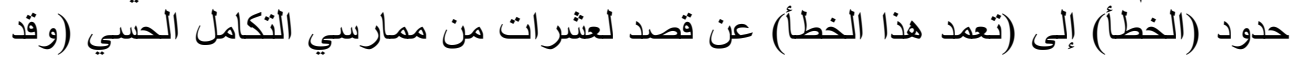

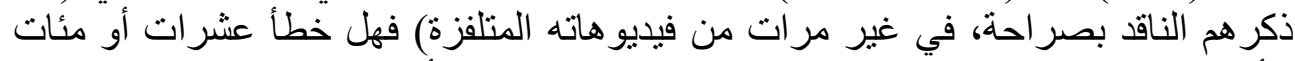

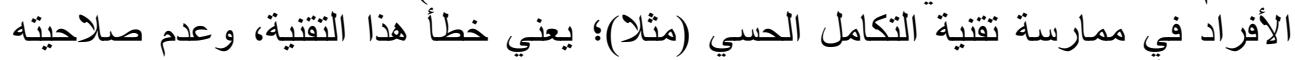

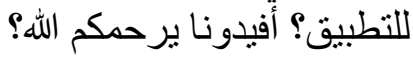
سؤال

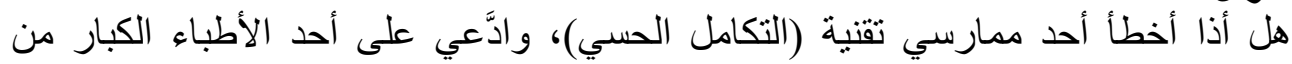

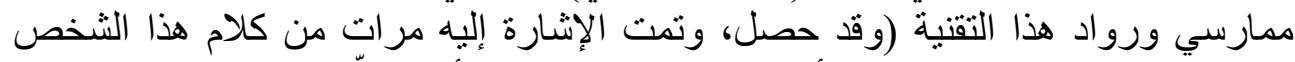

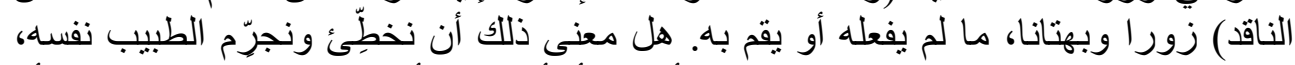

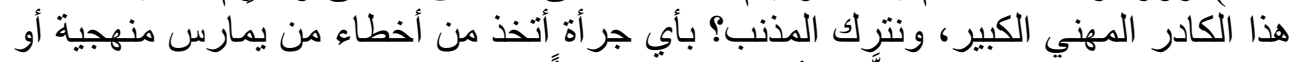

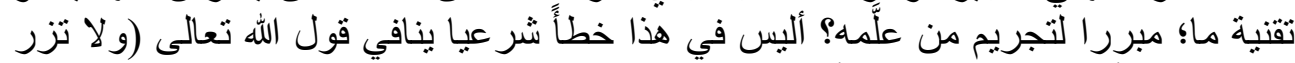

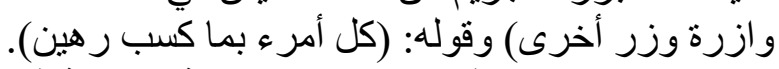

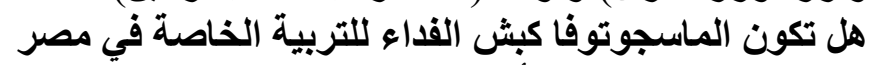

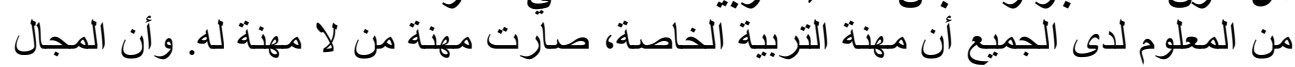

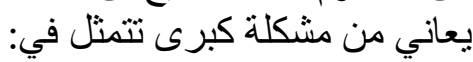

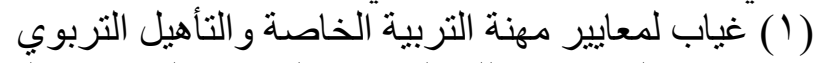

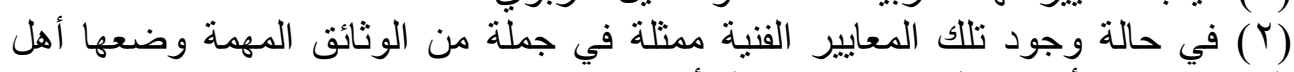

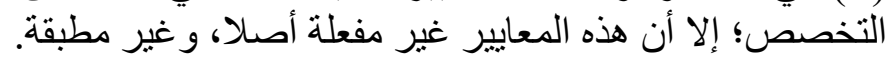

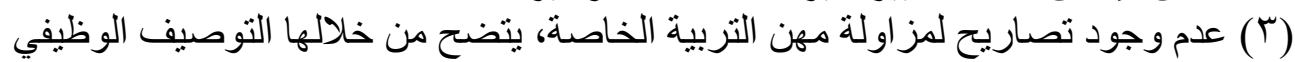

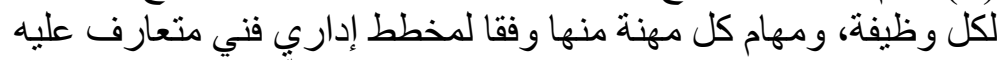

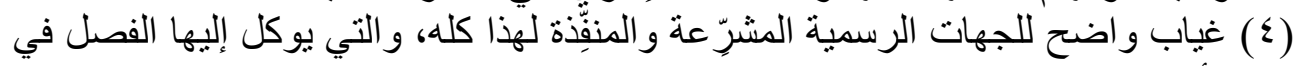
هذه الأمور.

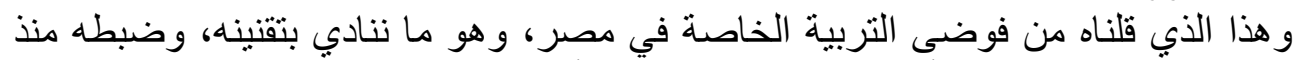

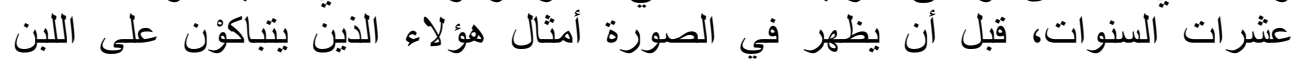

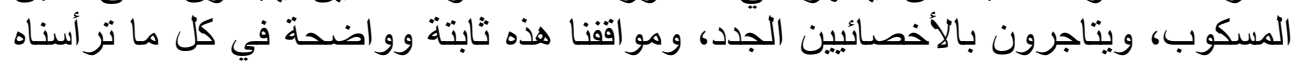

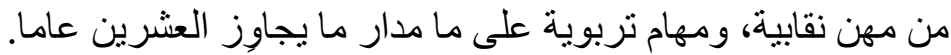

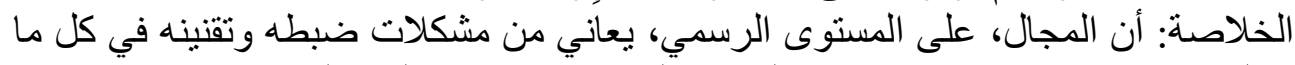

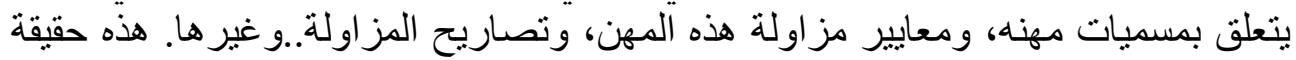


ظاهرة للعيان، وما زالت قضايا الإعاقة موزعة بين عدد من المؤسسات والوزارات ولم

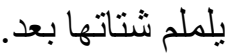
وبناء عليه:

- ماذا يعني أن يقوم شخص ما في وسط هذه اللخبطة المفاهيمية، المهنية ويقوم بتحميل

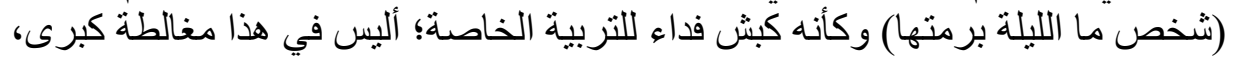

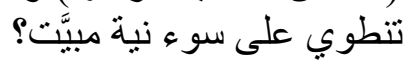

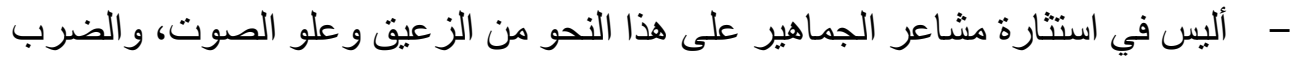

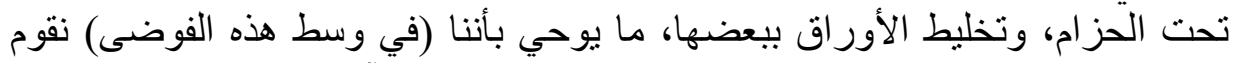
باعتلاء الموجه، و استثمار ها لصالحنا على النحو الذي يضر بآخرين؟ بأنا

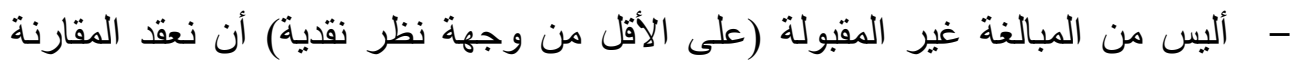

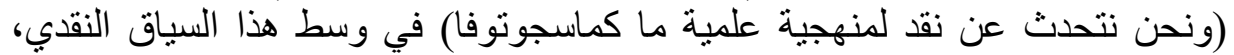

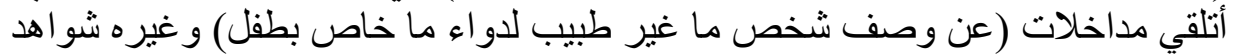

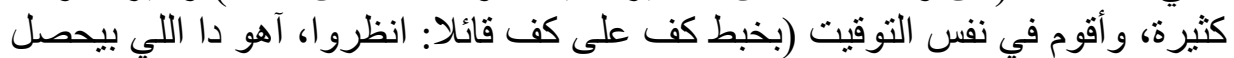

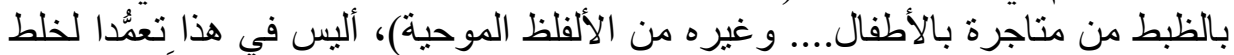

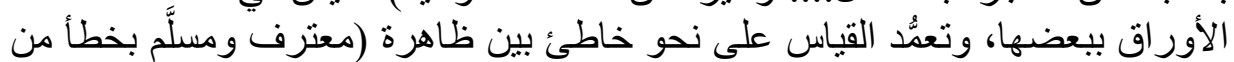

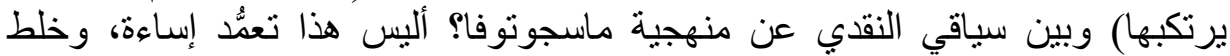

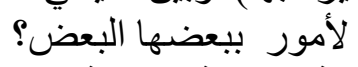

الثبهة التاسعة: طريقة النقا وأخلاقياته

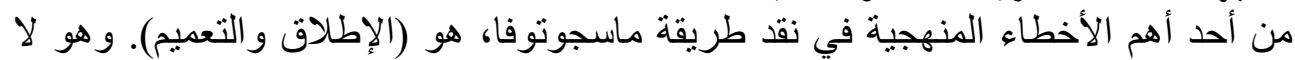

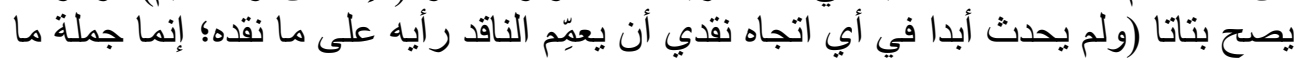

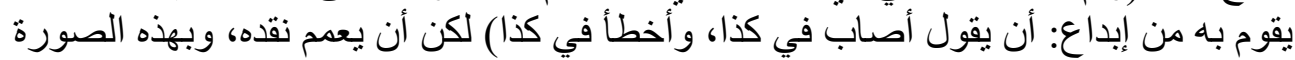

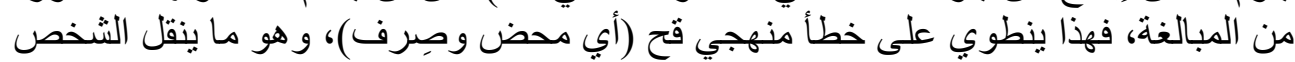

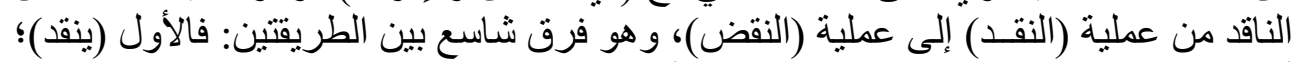

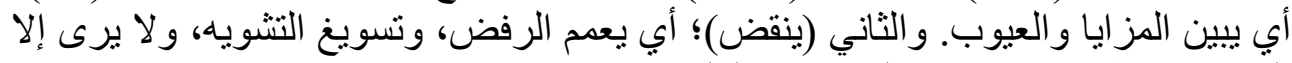

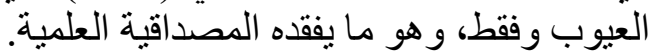
أما عن أوجه المبالغة و التعميم و الإطلاق في النقد، فيمكن أن نمثّل لها على النحو التالي:

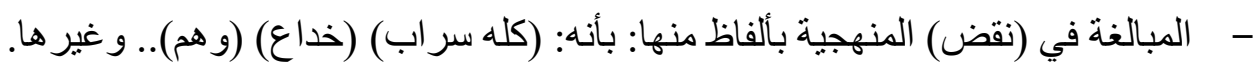
- - المبالغة في نقد أول من مارس و أدخلها في الوطن العربي بألفاظ لا تلايق.

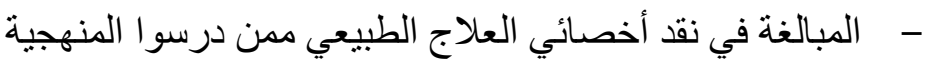

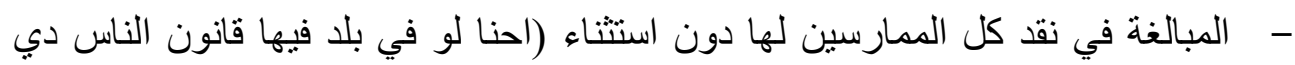
كانت اتعدمت). 


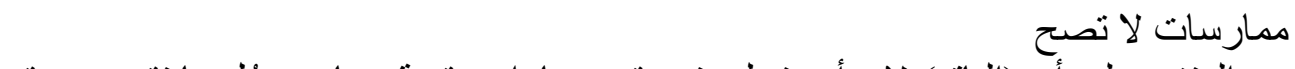

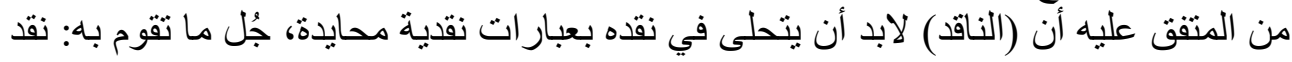
الطريقة و المحتوى لا الثخص (إلا إذا نقده من ناحية الممارسة المهنية) لا لا (سيرته الذاتية). لكن أن:

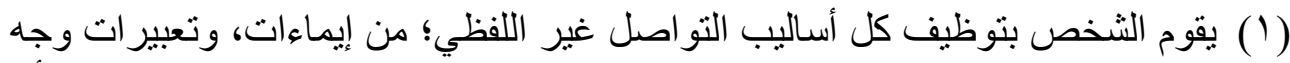

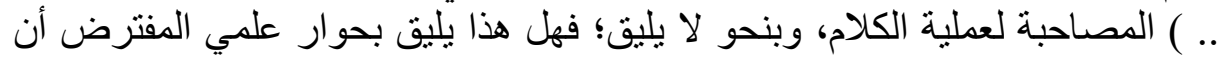
يكون منصفا.

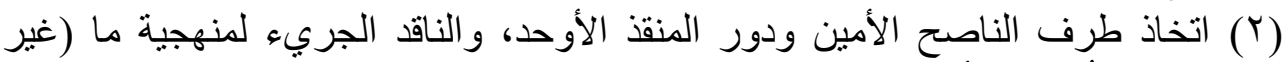

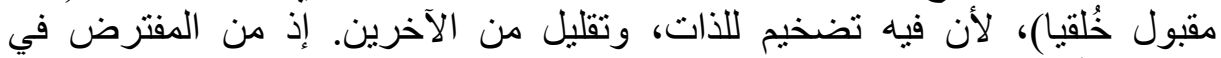

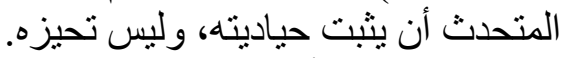

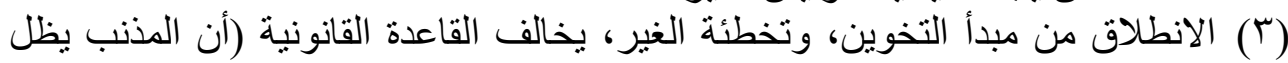
برئيا حتى تثبت إدانته).

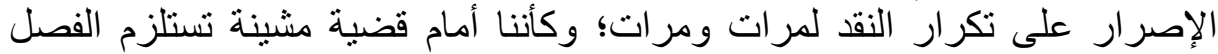

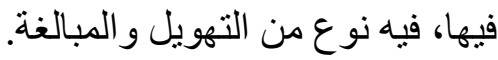
تعقيب واستفسار

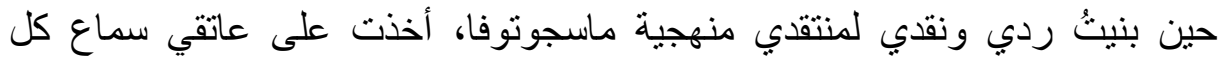

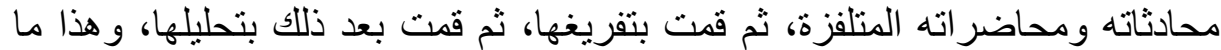
نعلمه من فنيات المنهج التحليلي النقدي.

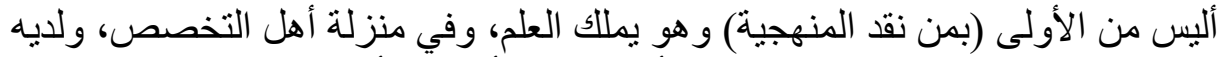

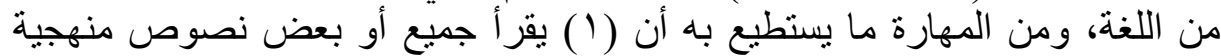

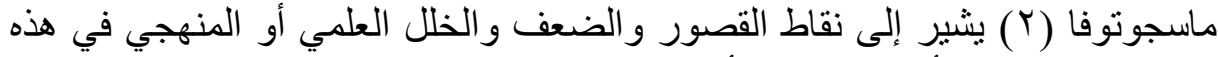

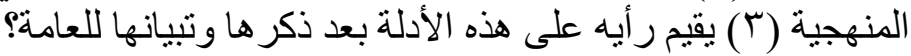

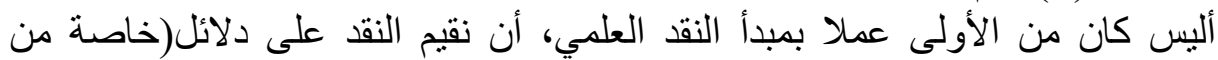

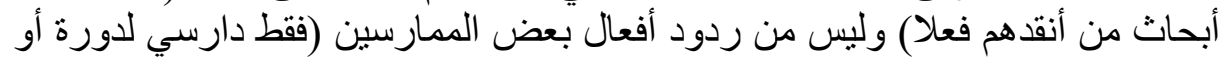

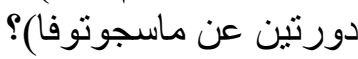
أليس كان من الأولى بدونا من نقان من العاملين و الممارسين لأي تقنية؛ أن ننقد التقنية نفسه (نقدا علميا تحليليا من منشور اته؟ 
مع الوصول إلى خاتمة الدطاف، أحب التنكير بأنني لم أرد على مدار الصفحات

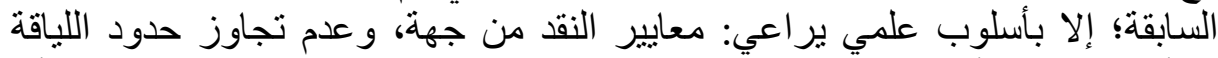

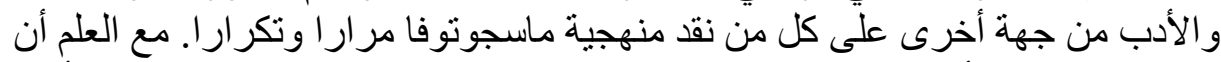

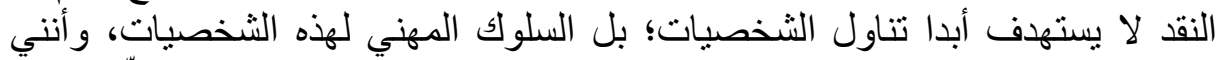

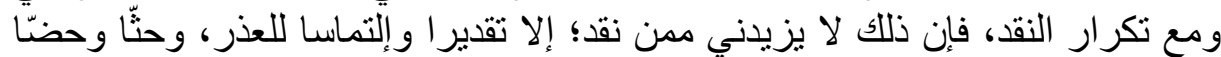

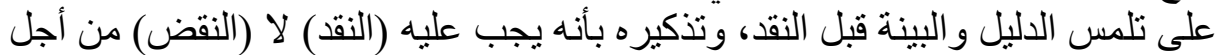
بنية علمية منهجية راسخة.

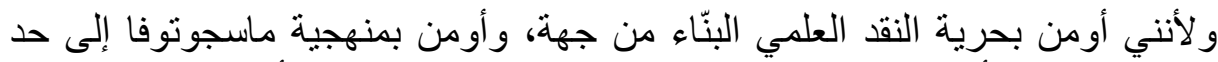

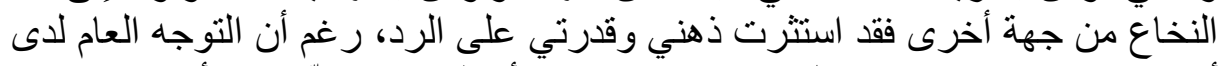

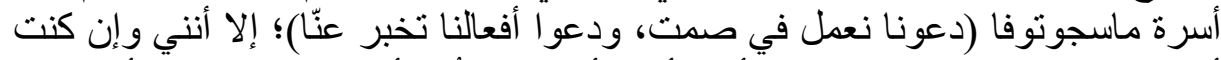

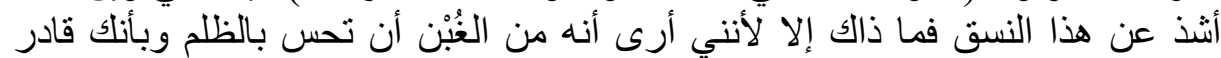

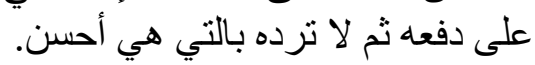

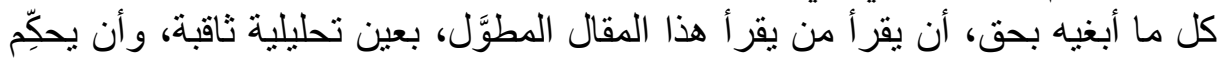

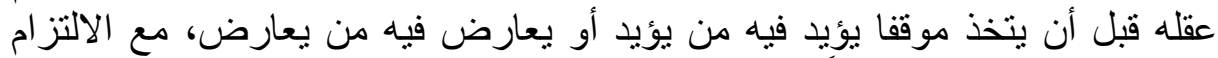

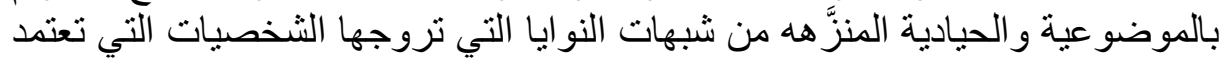
في مجمل أعمالها على الانفعال لا التعقل. 
Akhmatova, N. K., Masgutova, S., Sorokina, E., Akhmatov, E. A., \& Lebedinskaya, O. V. Influence of Neuro-Sensory-Motor Reflex Integration Technique on Immune Response of Patients with HerpesAssociated Multiforme Erythema. In Front. Immunol. Conference Abstract: IMMUNOCOLOMBIA2015-11th Congress of the Latin American Association of Immunology-10o. Congreso de la Asociación Colombiana de Alergia, Asma e Inmunología.

Akhmatov, E., Masgutov, D., Masgutova, S., \& Akhmatova, N. (2015). Neurophysiological Foundation of the MNRI ${ }^{\circledR}$ Reflex Integration Program

Allam, A. A., \& Abo-Eleneen, R. E. (2012). The development of sensorimotor reflexes in albino mice; albino rats and black-hooded rats. International Journal of Developmental Neuroscience, 30(7), 545553

Akhmatova, N. K. (2015). Immunological Effects of Masgutova Neurosensorimotor Reflex Integration in Children with Recurrent Obstructive Bronchitis. Int J Neurorehabilitation, 2(166), 2376-0281

Akhmatova, N. K., Masgutova, S., Lebedinskaya, O. V., Akhmatov, E. A., \& Shubina, I. (2015). Immunological Efficiency of MNRI Program at Treatment of Respiratory Diseases. In Front. Immunol. Conference Abstract: IMMUNOCOLOMBIA2015-11th Congress of the Latin American Association of Immunology-10o. Congreso de la Asociación Colombiana de Alergia, Asma e Inmunología. doi: 10.3389/conf. fimmu (Vol. 72).

Aulia, R., Kurniawati, D., \& Isnaini Herawati, S. (2017). Pengaruh Pemberian Tactile Stimulation Masgutova Neurosensorimotor Reflex Integration Dan Perceptual Motor Learning Terhadap Kemampuan Motorik Pada Anak Dengan Developmental Coordination Disorder (Doctoral dissertation, Universitas Muhammadiyah Surakarta). Amanor-Wilks, S. Stimulating the Autistic Brain with Motor Patterning and Childhood Reflexes.

Akhmatova, N., \& Akhmatova, E. (2017). Influence of MNRI on the Immune Status of Children with Down Syndrome. J Clin Cell Immunol, 7(483), 2. 
Bilbilaj, S., Aranit, G., \& Fatlinda, S. (2017). Measuring Primitive Reflexes in Children with Learning Disorders. European Journal of Multidisciplinary Studies, 5(1), 285-298.

Busz, T. E., \& Oginska-Dutkiewicz, B. (2015). Developmental Gains for a Child With Dyslexia and Allergies

Deiss, T., Meyers, R., Whitney, J., Bell, C., Tatarinova, T., Franckle, L., \& Beaven, S. (2019). Physiological Markers and Reflex Pattern Progression in Individuals with Neurodevelopmental Deficits Utilizing the MNRI Method. Neuroscience and Medicine, 10(1), 30-54.

Eve Kodiak, M. M. (2006). INFANT REFLEXES AND ADULT DEVELOPMENT Sensory Integration through Movement.

Koberda, J. L., \& Akhmatova, N. (2016). Masgutova Neurosensorimotor Reflex Integration (MNRI) as a New Form of Manual Neuromodulation Technique. J Neurol Neurobiol, 2(5).

Koberda, J. L., Akhmatova, N., Akhmatova, E., Bienkiewicz, A., Nowak, K., \& Nawrocka, H. (2016). Masgutova Neurosensorimotor Reflex Integration (MNRI) Neuromodulation Technique induces Positive Brain Amniotic (QEEG) Changes. J Neurol Neurobiol, 2(4).

Masgutova, S. (1990). Psychological Rehabilitation of Children-Victims of the Railway Catastrophe. Psychological Problems Journal, (1).

Masgutova, S. (1999). Effect of the Edu-K Exercises on the Work of Dynamic and Postural Reflexes. In Freiburger Kinesiologietage: Institute fur Angewandte Kinesiologie. Kinesiologie Kongress Deutschland. Freiburger

Masgutova, S. K. (2000). NeuroKinesiological basis of the reflex integration for successful learning of the child. In materials of Polish National Conference:"NeuroKinesiology as the Method of Facilitation of Development and Learning for Children and Youth with Dyslexia, ADHD, Autism and Asperger." 08.04. 2006.(P1.).

Masgutova, S. (2001). The influence of brain gym movements on the work of muscles and on dynamics and posture reflexes. Brain Gym Journal $X I, 1(2)$.

Masgutova, S., \& Akhmatova, N. (2004). Integration of dynamic and postural reflexes into the whole body movement system. Scientific edition: Prof. N. Akhmatova. MINK.(Pl.). Warsaw, 2007, 190. 
Masgutova, S. K., Ahmatova, N. K., \& Kolesnikov, A. (2004). Children with Challenges: Movement Development and Reflexes Integration. International NeuroKinesiology Institute.

Masgutova, S., \& Akhmatova, N. (2004). Reflexes re-patterning exercises. Integration of dynamic and postural reflexes into the whole body movement system.

Masgutova, S., \& Akhmatova, N. (2004). The integration of dynamic and postural reflexes of the entire mobility system. MINK, Warsaw, Poland.

Masgutova, S., \& Akhmatova, N. (2004). Children with Challenges: Integration of Dynamic and Postural Reflexes. MINK, Warsaw.

Masgutova, S. (2005). Reflexes as the Basis of the Nerve System Development and Formation of the Motor Patterns in Infancy. Materials of International Conference: Modern Methods of Stimulation of Motor and Language Development. International Kinesio-Rehabilitation Camp for Children with Challenges of Dr. S. Masgutova Institute, MINK. Warsaw

Masgutova, S. K., Kowal, J., Mazur, G., \& Masgutov, D. P. (2005). NeuroKinesiology tactile therapy ${ }^{\mathrm{TM}}$. Scientific Edition: Dr. A. Regner. J. Szymczak. Warszawa: MINK.

Masgutova, S., \& Sadowska, L. (2006). The Use of the Edu-K for Children with Learning Difficulties: Early Developmental Dynamic. In materials of: Polish National Conference: "NeuroKinesiology as the Method of Facilitation of Development and Learning for Children and Youth with Dyslexia, ADHD, Autism and Asperger (Vol. 8, No. 2006, pp. 39-50).

Masgutova, S. K. (2006). Neuro-Kinesiology as the support for development and learning. Children with Learning Challenges and ADHD. In Materials of Polish National Conference: "Modern Methods of Stimulation of Movement Development and Learning in Children with Difficulties in Learning, ADHD and Autism (Vol. 29, pp. 236-253).

Masgutova, S. K. (2006). Infant reflex integration in movement development. In Material s of Polish National Conference: Modern Methods of Stimulation of Movemen $t$ Development and Learning in Children with Difficulties in Learning, ADHD an d Autism. Poland. Pp (pp. 5-28). 
Masgutova, S. (2007). Integration of Infant Dynamic and Postural Reflex

Patterns: Masgutova Neuro-Sensory-Motor and Reflex IntegrationMNRI $^{\mathrm{TM}}$ Method for Children and Adults. SMEI, Orlando, USA.

Masgutova, S. (2015). MNRI® for Children with Cerebral Palsy.

MasgutovaS.(2015).MNRI®:TheNeurosensorimotor Reflex Integration to

Promote Learning and Neurodevelopment

Masgutova, S., \& Masgutov, D. (2015). MNRI® assessment for determining the level of reflex development. Reflexes: Portal to Neurodevelopment and Learning: A Collective Work, Svetlana Masgutova Educational Institute, Orlando, 201.

Masgutova, S., Akhmatova, N., \& Ludwika, S. (2016). Reflex Profile of Children with Down Syndrome Improvement of Neurosensorimotor Development Using the MNRI® Reflex Integration Program. Int $J$ Neurorehabilitation, 3(197), 2376-0281.

Masgutova, S., Akhmatova, N., Sadowska, L., Shackleford, P., \& Akhmatov, E. (2016). Progress with neurosensorimotor reflex integration for children with Autism Spectrum Disorder. Journal of neurology and psychology, 4(2), 14.

Masgutova, S. (2016). The questionnaire of dynamic changes in children's abilities. Masgutova Foundation.

Masgutova, S. (2007). Integration of Infant Dynamic and Postural Reflex Patterns: Neuro-Sensory-Motor and Reflex Integration Method for Children and Adults. Poland: Ascent Educational Institute Publishing Company.

Masgutova, S. K. (2007). Neuro-Sensory-Motor Development: Visual and Auditory Reflexes Integration. Facilitation Program of Development and Learning for Children and Adults. Warsaw, Poland.

Masgutova, S. (2007). Neuro-structural reflex integration therapy.

Masgutova, S. K., Akhmatova, N. K., \& Kiselevsky, M. V. (2008). Clinicimmunological assessment of effect of the therapy program of neurosensory-motor integration of reflex patterns at chronic inflammatory diseases of respiratory system. Russ Immun J, 2, 454-463.

Masgutova, S. K., \& Regner, A. (2008). Language development using sensory-motor integration approach. Scientific edition: Prof. $T$. Galkowski, Dr. B. Dolyk. Warsaw: Continulo. 
Masgutova, S. (2008). Masgutova method of reflex integration for children with cerebral palsy. Svetlana Masgutova Educational Institute ${ }^{\circledR}$ for Neuro-Sensory-Motor and Reflex Integration, SMEI (USA) Posjećeno, 15, 2016.

Masgutova, S., Russia-poland, P. D., Wenberg, E. S., \& Retschler, M. (2008). Masgutova Method of Reflex Integration for Children With Cerebral Palsy, 1-23.

Masgutova, S. K., \& Akhmatova, N. K. (2008). Repatterning and integration of dysfunctional and pathological reflexes.

Masgutova, S. (2009). MNRI® Neurosensorimotor Development: Visual and Auditory Reflexes Integration. Facilitation Program of Development and Learning for Children and Adults. Florida, 86.

Masgutova SK, Akhmatova NK, Sadowska L, Shackleford P and Akhmatov EA(2016) Neurosensorimotor Reflex Integration for Autism: aNew Therapy Modality Paradigm, Journal of Pediatric Neurological Disorders, Volume $2 \cdot$ Issue 1

Madison, C., Marquardt, V., Miller, B., Mueller, A., Pearce, S., Philion, C., ... \& Stainbrook, A. (2016). Sensory/Manipulation Interventions For Children with Autism and Developmental Disabilities: An EvidenceBased Practice Project.

Masgutova, S. K., Shackleford, P., \& Masgutov, D. R. (2017). The Use of Restoring Resources of the Survival Roles and Reflex Patterns in

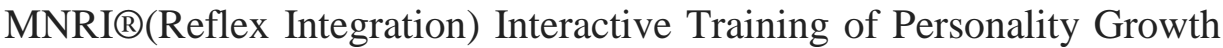
and Interpersonal Relations. Social Psychology \& Society, 8(4).

Masgutova, S., \& Regner, A. (2009). The development of child's speech in the light of sensory integration. Continuo, Wrockaw, Poland.

MASGUTOVA, S. (2010). Integration of Infant Dynamic and Postural Reflex Patterns-MNRI. USA: Masgutova Educational Institute.

Masgutova, S. (2011). Infant dynamic and postural reflexes. Neuro-sensorymotor reflex integration.

Masgutova, S., \& Denis, M. (2012). MNRI® Oral-facial reflex integration in children and adults with neurodeficits (Polish version). Warsaw, Poland: ISME.

Masgutova, S. K., Akhmatova, N. K., \& Lebedinskaya, O. V. (2013). Clinical-immunological assessment of therapy effect of the neuro- 
sensory-motor integration program of reflex patterns in airway chronic inflammatory diseases. In International Congress in Milan, Front Immuno $l$ Conference Abstract: 15th International Congress of Immunology (ICI).

Masgutova, S., \& Masgutov, D. (2015). MNRI® assessment for determining the level of reflex development. book: Reflexes: Portal to Neurodevelopment and Learning. A Collective Work. SMEI, Florida, USA.

Masgutova, S., Masgutov, D., Akhmatova, N., \& Akhmatov, E. (2015). Neurophysiological Foundation of the MNRI ${ }^{\circledR}$ Reflex Integration Program. A Collective Work. Florida, USA: SMEI, 31-40.

Masgutova, S. (2015). Reflex Integration for Post-Trauma Survival an d Recovery. A Collective Work. Florida, USA: SMEI, 70-91.

Masgutova, S., \& Masgutov, P. D. D. (2015). A Search for Excellence in Gifted Children with Reflex Integration.

Masgutova, S., \& Masgutov, D. (2015). Reflex Integration for Trauma Recovery MNRI ${ }^{\circledR}$ Stress Resilience Introductory Program. Orlando: SMEI.

Masgutova, S., \& Masgutov, D. (2015). Reflex Integration Disorder as a Ne w Treatment Paradigm for Children with Autism. A Collective Work. Florida, USA: SMEI, 171-180

Nowak, K., Sobaniec, P., Sobaniec, W., Akhmatova, N., \& Shackleford, P. (2020). Evaluation of the Therapeutic Effect of MNRI Reflex Neuromodulation on Children Diagnosed with Autism Based on Reflex Assessments, QEEG Analysis and ATEC Questionnaire. J Neurol Neurobiol, 6(2).

Nussbaum, S. S. (2010). The effects of 'Brain Gym'as a general education intervention: Improving academic performance and behaviors. Northcentral University.

Ortego, L., Pelican, E., \& Callaba, L. (2015). An Investigation of the Effects of MNRI ${ }^{\circledR}$ Techniques on the Educational Performance of Kindergarten Students.

Pilecki, W., Masgutova, S., \& Kowalewska, J. e t al.(2012) The impact of rehabilitation carried out using the Masgutov a neurosensorimotor reflex 
integration method in children with cerebral palsy on the results of brain stem auditory potential examinations. Adv Clin Ex p Med, 21, 363-371. Pilecki, W., Kipiński, L., Szawrowicz-Pełka, T., Kałka, D., \& Masgutova, S. (2013). Spectral brain mapping in children with cerebral palsy treated by the Masgutova Neurosensorimotor Reflex Integration method. Journal of the Neurological Sciences, 333, e550.

Pilecki, W., Masgutova, J. K., Masgutov, D. N. A., \& Sobieszczanska, M. (2012). The Impact of the MNRI® Program on the Brain Stem Auditory Potential in Children with Cerebral Palsy. Journal of Wroclaw Medical Academy, 3.

Pilecki, W., Kipiński, L., Szawrowicz-Pełka, T., Kałka, D., \& Masgutova, S. (2013). Spectral brain mapping in children with cerebral palsy treated by the Masgutova Neurosensorimotor Reflex Integration method. Journal of the Neurological Sciences, 333, e550.

Pilecki, W., Pilecka-Kalamarz, A., Kalka, D., \& Kipinski, L. (2015). EEG

Mapping Shows Changes in Brainwave Spectrum in Children with Cerebral Palsy During and After Masgutova Neurosensorimotor Reflex Integration Therapy.

Rentschler, M. M., Method, M., \& Specialist, I.(2008). The Masgutova

Method of Neuro-Sensory-Motor and Reflex Integration: Key to Health. Development and Learning,(202).

Rentschler, M. (2008). The Masgutova Method of Neuro-Sensory-Motor and Reflex Integration: Key to Health, Development and Learning. Posjećeno, 15(4), 2016.

Shackleford, P. (2015). MNRI®-Neurotypical Development and Reflex Integration Disorder.

Svetlana Masgutova, Ludwika Sadowska, Joanna Kowalewska, Denis Masgutov, Nelli Akhmatova and Henryk Filipowski (2015). Use of a Neurosensorimotor Reflex Integration Program to Improve Reflex Patterns of Children with Down Syndrome, Journal-Of-Neurology- No. $4: 59$

Thelen, A., Tiedt, E. M., Vang, S. C., Wagle, P. L., Weber, L. K., Wood, J. T., ... \& Zimmerli, B. M. (2016). Movement Interventions for Children with Autism and Developmental Disabilities An Evidence-Based Practice Project 
Renard-Fontaine, I. (2017). Effect of Reflex Neuromodulation on an Infant with Severe Amniotic Band Syndrome: A Case Report on the Use of MNRI Techniques for Physical Therapy. Int. J. Neurorehabilit, 4, 248.

Masgutova, S. (2018). Post-Trauma Recovery in Children of Newtown, CT using MNRI Reflex Integration. Journal of Traumatic Stress Disorders \& Treatment, 2017.

Masgutova, S., Masgutov, D., \& Lieske, T. (2018). Effects of MNRI Visual Reflex Neuro-Training on Visual and Academic Skills of Children with Autism. Int J Sch Cogn Psychol, 5(213), 2.

Katarzyna Nowak- Halina Muzaj- Halina Nawrocka-Piotr Sobaniec,(2018) Evaluation of the effectiveness of MNRI therapy (Masgutova neurosensorimotor reflex integration) in children with autism based on the ATEC (autism treatment evaluation checklist) test - a preliminary report

Tatarinova, T. V., Deiss, T., Franckle, L., Beaven, S., \& Davis, J. (2020). The impact of MNRI therapy on the levels of neurotransmitters associated with inflammatory processes. International journal of molecular sciences, 21(4), 1358. 